Florida International University FIU Digital Commons

$11-3-2011$

\title{
Optimum Wireless Power Transmission for Sensors Embedded in Concrete
}

Shan Jiang

Florida International University, sjian002@fiu.edu

DOI: $10.25148 /$ etd.FI12120201

Follow this and additional works at: https://digitalcommons.fiu.edu/etd

\section{Recommended Citation}

Jiang, Shan, "Optimum Wireless Power Transmission for Sensors Embedded in Concrete" (2011). FIU Electronic Theses and Dissertations. 549.

https://digitalcommons.fiu.edu/etd/549 


\section{FLORIDA INTERNATIONAL UNIVERSITY}

Miami, Florida

\section{OPTIMUM WIRELESS POWER TRANSMISSION FOR SENSORS EMBEDDED IN CONCRETE}

A dissertation submitted in partial fulfillment of the

requirements for the degree of

DOCTOR OF PHILOSOPHY

in

ELECTRICAL ENGINEERING

by

Shan Jiang

2011 
To: Dean Amir Mirmiran

College of Engineering and Computing

This dissertation, written by Shan Jiang, and entitled Optimum Wireless Power Transmission for Sensors Embedded in Concrete, having been approved in respect to style and intellectual content, is referred to you for judgment.

We have read this dissertation and recommend that it be approved.

Osama A. Mohammed

Jean H. Andrian

Stavros V. Georgakopoulos, Major Professor

Date of Defense: November 3, 2011

The dissertation of Shan Jiang is approved.

Dean Amir Mirmiran College of Engineering and Computing

Dean Lakshmi N. Reddi University Graduate School

Florida International University, 2011 
(C) Copyright 2011 by Shan Jiang

All rights reserved. 


\section{DEDICATION}

I dedicate this dissertation to my family. Without their love, patience, understanding and support, the completion of this work would never have been possible. 


\section{ACKNOWLEDGMENTS}

I would like to express my sincerest gratitude to my major professor, Dr. Stavros V. Georgakopoulos, for his excellent guidance and support through my $\mathrm{Ph}$. D. stage at FIU. He provided me the most convenient research environment including tremendous constructive academic advice, many studious lab colleagues and the latest research tools, which ensured the quality of my education and research. His hard-working, gentle and earnest way of life and research sets up a role model for me, and I sincerely thank him for his help and patience during these three important years of my life. I also appreciate Dr. Osama A. Mohammed, Dr. Jean H. Andrian, and Dr. Berrin Tansel for serving on my dissertation defense committee and giving enlightening comments.

I am extremely grateful to my family members who supported me throughout the research. Without my husband's love and company, my father's encouragement and my mother's advice, it would have been difficult for me to conduct this research.

I sincerely thank all the members of FIU Electrical Engineering Department and Telecommunications and Information Technology Institute for their work and dedication, special thanks to Olutola Jonah, Yipeng Qu, Oscar Silveira, Dr. Qian Wang, Dr. Kai Chen, and Hao Hu for their help and friendship.

Finally, I would like to greatly thank the Graduate School of FIU for providing to me the Dissertation Year Fellowship, which supported this research. 


\title{
ABSTRACT OF THE DISSERTATION \\ OPTIMUM WIRELESS POWER TRANSMISSION FOR SENSORS \\ EMBEDDED IN CONCRETE
}

\author{
By
}

Shan Jiang

Florida International University, 2011

Miami, Florida

\section{Professor Stavros. V. Georgakopoulos, Major Professor \\ Various nondestructive testing (NDT) technologies for construction and} performance monitoring have been studied for decades. Recently, the rapid evolution of wireless sensor network (WSN) technologies has enabled the development of sensors that can be embedded in concrete to monitor the structural health of infrastructure. Such sensors can be buried inside concrete and they can collect and report valuable volumetric data related to the health of a structure during and/or after construction. Wireless embedded sensors monitoring system is also a promising solution for decreasing the high installation and maintenance cost of the conventional wire based monitoring systems. Wireless monitoring sensors need to operate for long time. However, sensor batteries have finite life-time. Therefore, in order to enable long operational life of wireless sensors, novel wireless powering methods, which can charge the sensors' rechargeable batteries wirelessly, need to be developed.

The optimization of RF wireless powering of sensors embedded in concrete is studied here. First, our analytical results focus on calculating the transmission loss and propagation loss of electromagnetic waves penetrating into plain concrete at different 
humidity conditions for various frequencies. This analysis specifically leads to the identification of an optimum frequency range within $20-80 \mathrm{MHz}$ that is validated through full-wave electromagnetic simulations. Second, the effects of various reinforced bar configurations on the efficiency of wireless powering are investigated. Specifically, effects of the following factors are studied: rebar types, rebar period, rebar radius, depth inside concrete, and offset placement. This analysis leads to the identification of the 902$928 \mathrm{MHz}$ ISM band as the optimum power transmission frequency range for sensors embedded in reinforced concrete, since antennas working in this band are less sensitive to the effects of varying humidity as well as rebar configurations. Finally, optimized rectennas are designed for receiving and/or harvesting power in order to charge the rechargeable batteries of the embedded sensors. Such optimized wireless powering systems exhibit significantly larger efficiencies than the efficiencies of conventional RF wireless powering systems for sensors embedded in plain or reinforced concrete. 


\section{TABLE OF CONTENTS}

CHAPTER

PAGE

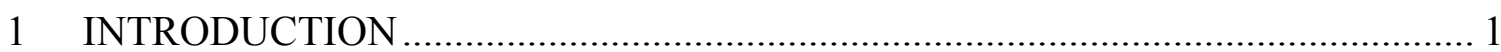

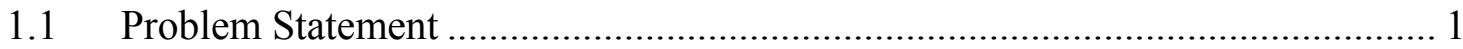

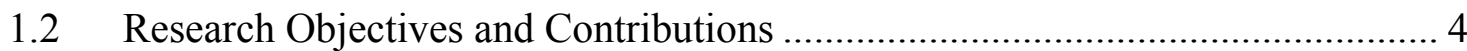

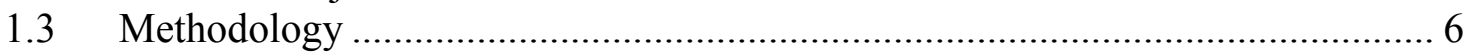

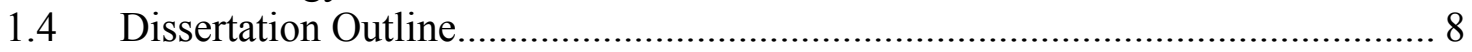

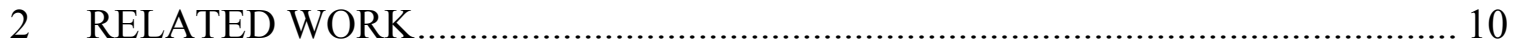

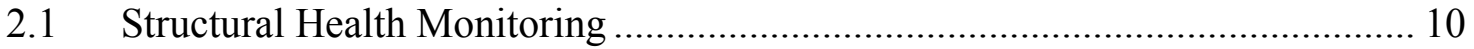

2.2 Wireless Sensor Network for SHM Applications ........................................... 12

2.3 Wireless Power Transmission for Embedded SHM Sensors ............................ 15

2.3.1 Piezoelectric Converter ................................................................... 17

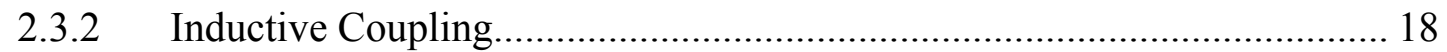

2.3.3 Strong Resonant Coupling …………….............................................. 19

2.3.4 Electromagnetic Radiation.................................................................. 20

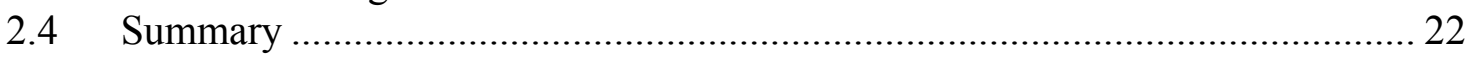

3 PLANE WAVE MODEL FOR PLAIN CONCRETE ………………………........ 24

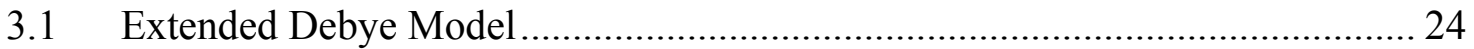

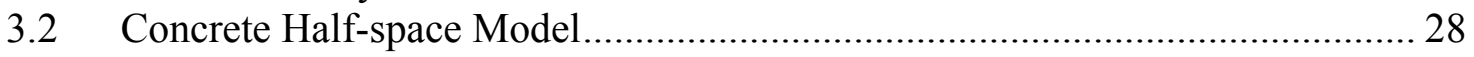

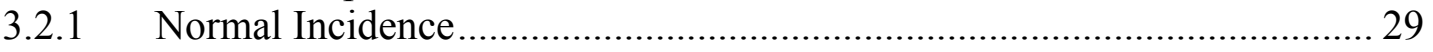

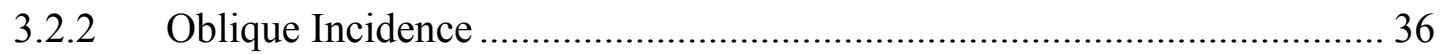

3.3 Concrete Slab Model.............................................................................. 43

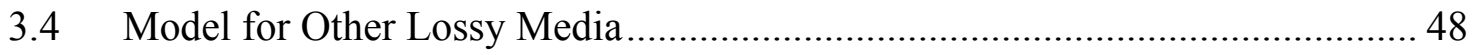

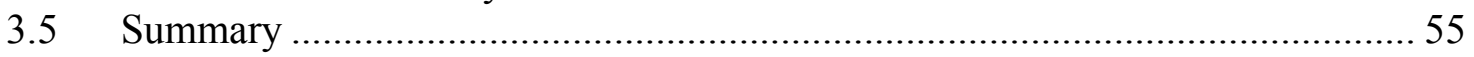

4 PLANE WAVE MODEL FOR REINFORCED CONCRETE ……………….......... 57

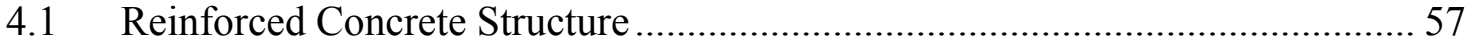

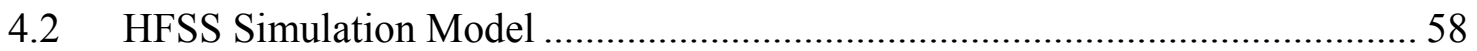

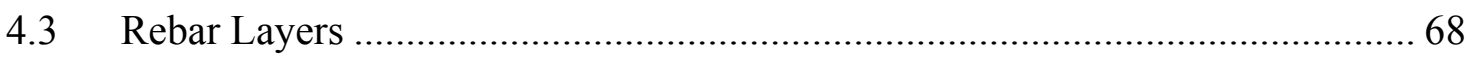

4.4 Reinforced Concrete Slab...................................................................... 74

4.4.1 Simulation Model........................................................................... 74

4.4.2 Effects of Concrete Slab ...................................................................... 75

4.4.3 Effects of Rebar Configuration.............................................................. 80

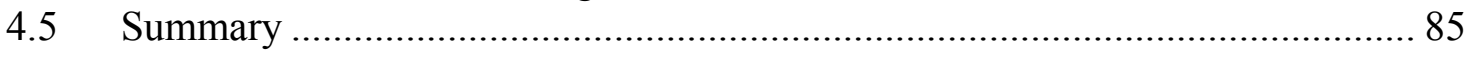

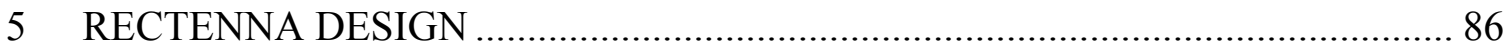

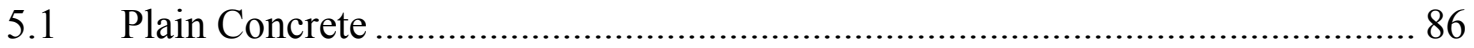

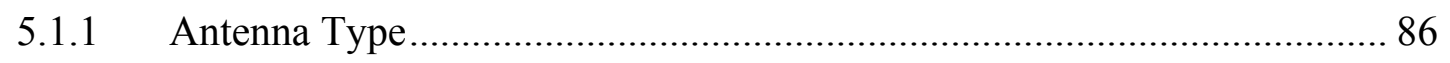

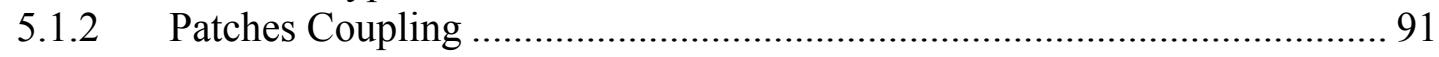

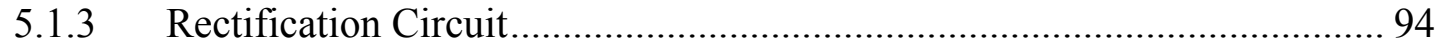

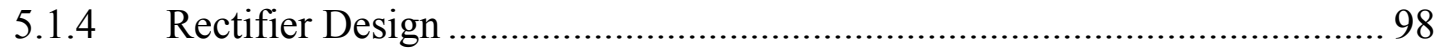




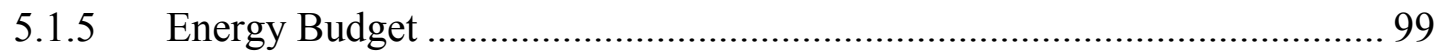

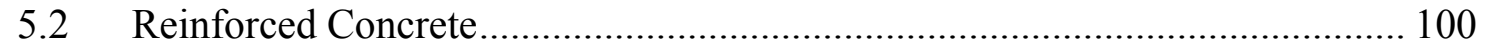

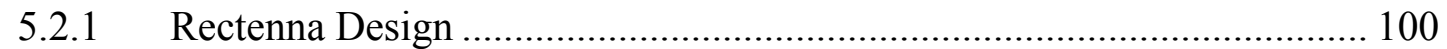

5.2.2 Near Field Coupling Solutions ............................................................ 103

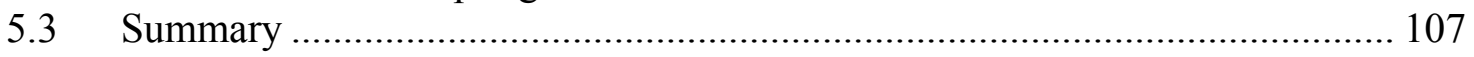

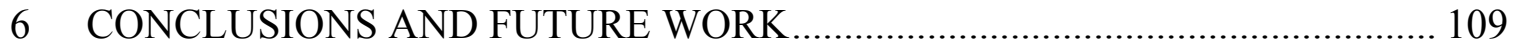

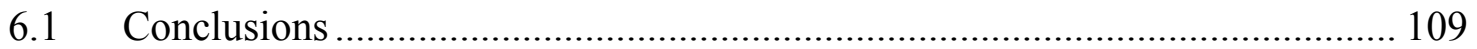

6.2 Future Work ....................................................................................... 111

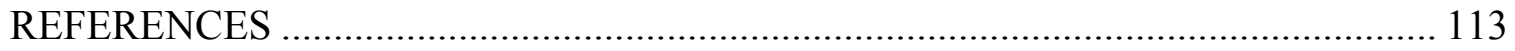

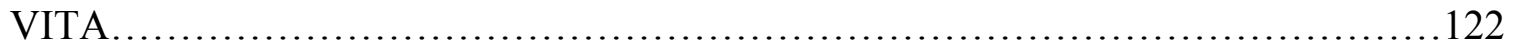




\section{LIST OF FIGURES}

FIGURE

PAGE

Figure 2.1: Configurations for sensor monitor system. ....................................... 13

Figure 3.1: Permittivity of concrete versus frequency........................................... 27

Figure 3.2: Effective conductivity of concrete versus frequency. ............................... 28

Figure 3.3: Plane wave penetrating concrete half-space at normal incidence................. 29

Figure 3.4: Transmission loss for normal incidence at different humidity conditions..... 32

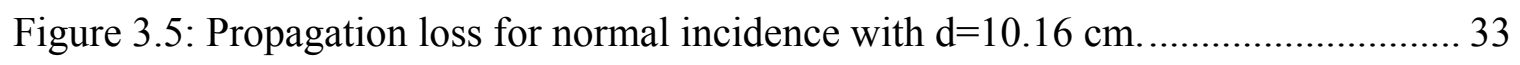

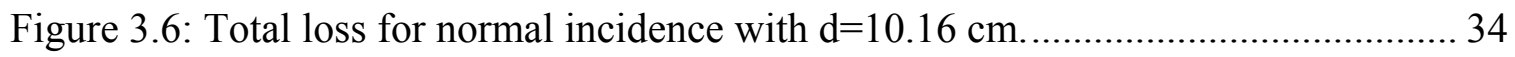

Figure 3.7: Total loss versus frequency and propagation depth. ............................... 35

Figure 3.8: Plane wave penetrating concrete half-space at oblique incidence. ............... 38

Figure 3.9: Transmission coefficient for oblique incidence at $50 \mathrm{MHz}$........................ 39

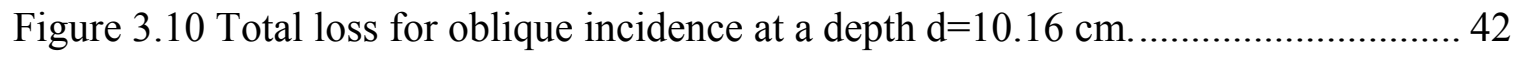

Figure 3.11: Plane wave penetrating concrete slab at normal incidence...................... 43

Figure 3.12: Transmission and reflection coefficients for plane wave penetrating a

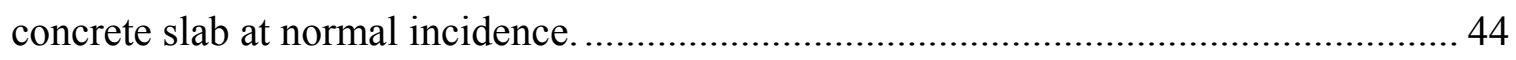

Figure 3.13: Total loss for normal incidence with $\mathrm{d} 1=10.16 \mathrm{~cm}, \mathrm{~h}=12 \% \ldots \ldots \ldots \ldots \ldots \ldots \ldots . . . . . \ldots 7$

Figure 3.14: Total loss for plane wave propagating from air to fresh water. ................. 51

Figure 3.15: Total loss for plane wave penetrates through air to soil........................... 54

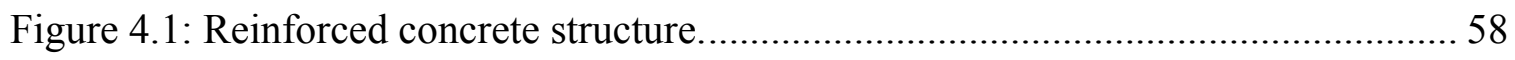

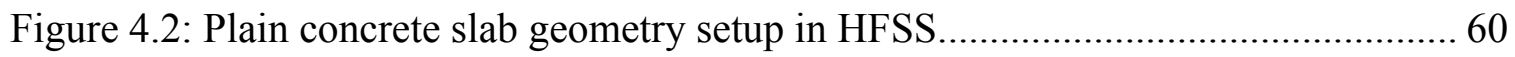

Figure 4.3: Plain concrete boundary condition setup in HFSS ................................. 61

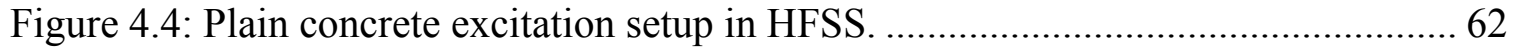


Figure 4.5: Plain concrete solution setup in HFSS. 64

Figure 4.6: Comparison of simulated and theoretical results. .................................. 66

Figure 4.7: Reflection and transmission coefficients for plain concrete slab................. 67

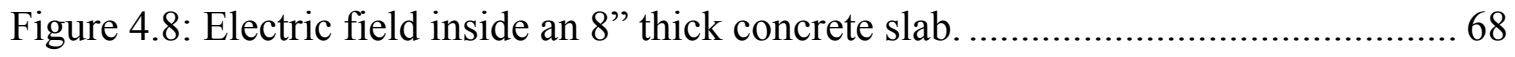

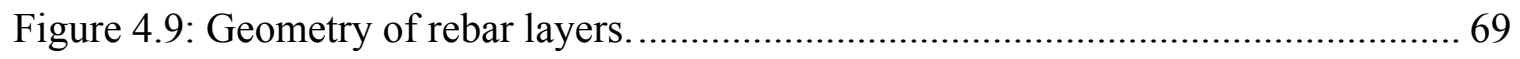

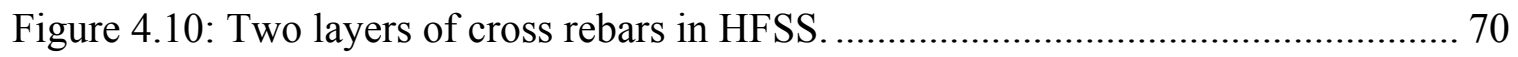

Figure 4.11: Reflection and transmission coefficients for two layers cross rebars. ........ 71

Figure 4.12: Electric field distribution for two layers cross rebars............................. 73

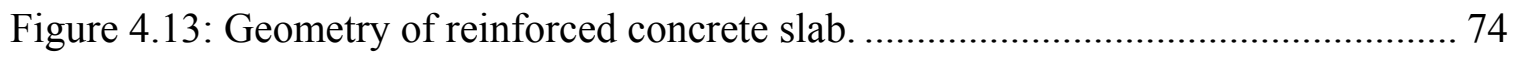

Figure 4.14: Reinforced concrete simulation setup in HFSS ................................... 75

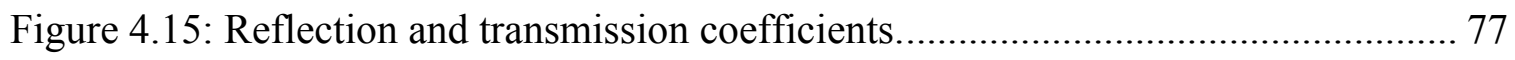

Figure 4.16: Maximum electric field induced inside reinforced concrete slab............... 79

Figure 4.17: Reflection and transmission coefficients for reinforced concrete slab with

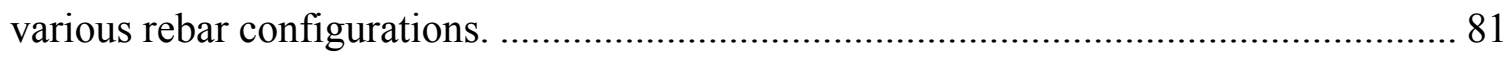

Figure 4.18: Offset between the two mesh layers inside reinforced concrete slab.......... 83

Figure 4.19: Reflection and transmission coefficients for reinforced concrete slab with

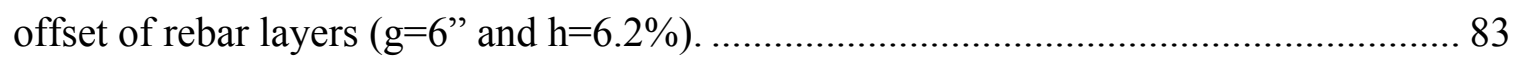

Figure 4.20: Maximum electric field induced inside reinforced concrete slab for various

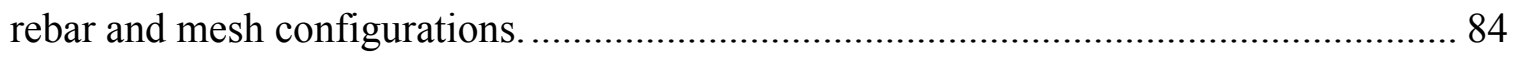

Figure 5.1: Simulation setup for two dipoles coupling in air-concrete slab model......... 87

Figure 5.2: Computed S-parameters of two dipoles coupling in free space, dry concrete

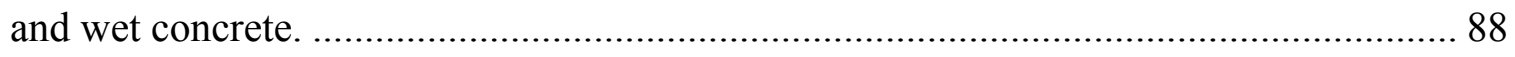

Figure 5.3: Simulation setup for two patches coupling in air-concrete slab model......... 89 
Figure 5.4: Computed S-parameters of two patches coupling in free space, dry concrete and wet concrete. 90

Figure 5.5: Simulation setup of two patches in the air-concrete model.......................... 93

Figure 5.6: Patches coupling for different concrete humidity conditions....................... 93

Figure 5.7: Patches coupling for different resonant frequencies. ................................ 94

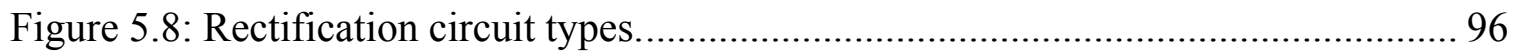

Figure 5.9: Wireless powering system setup with voltage doubler............................ 99

Figure 5.10: Wireless powering system setup with matched voltage doubler................ 99

Figure 5.11: Simulation setup for two antennas in the air-reinforced concrete model... 101

Figure 5.12: Computed S-parameters of two antennas coupling.............................. 102

Figure 5.13: Simulation setup for three rebar types.............................................. 105

Figure 5.14: Patches coupling for three rebar types. ............................................. 107 


\section{CHAPTER 1}

\section{INTRODUCTION}

\subsection{Problem Statement}

Various nondestructive testing (NDT) technologies for construction and performance monitoring have been studied for decades with increasing interest. However, NDT technologies that are based on wireless solutions, only recently become the subject of industry and research work in the context of concrete structural health monitoring (SHM). The increased interest stems from both current society safety expectations, and recent technological developments in the industries of concrete, sensors, microelectronics, and wireless communications. Especially, in the past few years there have been several incidents of large structures, buildings, dams, bridges and airport infrastructures collapsing without warning that have caused significant financial losses and great loss of life. For example, Minnesota's fifth busiest bridge, I-35W Mississippi River Bridge suddenly collapsed during the evening rush hour in 2007, killing 13 people and injuring 145 people. These events unfortunately have happened without any warning and without an opportunity for engineers to intervene and attempt to correct each situation. Such events have raised the public's awareness regarding the safety of large public infrastructure. Also, our society has realized that we need technologies that can predict tragic events before they have catastrophic consequences. One way to predict such events is through constant monitoring of structures. One promising solution relies on sensors that can measure and report certain physical quantities of the structures, such as humidity, force, stress, displacement, etc [1]. However, the majority of mature technologies rely on sensors that are connected through wires to a central station that provides power to the 
sensors and collects their data. The installation of such sensor systems is expensive and labor intensive. In fact, the installation of wires represents up to $25 \%$ of the total system cost with over $75 \%$ of the installation time [2]. Also, if the wires connected to sensors get corroded then these sensors become inoperative. Therefore, it is imperative that new technologies are developed for monitoring the health of structures while providing minimal installation costs and reliable operation.

A promising solution is based on the use of wireless sensors that communicate wirelessly as well as receive their power remotely without being connected to any wires, so the installation time and cost can be significantly reduced. The tasks of wireless SHM can be classified into the following two groups. The first group involves monitoring structures based on data that can be obtained from sensors that are placed or mounted on the surface of the structures. These sensors can read: surface temperature, vibration, displacement, noise, etc. The second group involves structural monitoring based on data obtained from sensors embedded inside concrete, that can read volumetric data, such as temperature, $\mathrm{pH}$, strain, rebar corrosion, crack, pressure, etc. It should be pointed out that volumetric characteristics can describe the health of a structure more accurately. Therefore, wireless embedded sensors are more suitable for SHM applications. Furthermore, wireless embedded sensors will enable monitoring of building materials and processes during the construction period, so that structures are built with greater quality controls thereby ensuring greater reliability for structures. For example, when building hi-rises with high strength concrete, it is crucial that concrete is properly cured before construction proceeds to the next floor to ensure full and proper strength of the concrete. However, there is no deterministic way to decide when the concrete has cured unless we 
can directly monitor the concrete properties, such as, $\mathrm{pH}$ and moisture content. In such situations, sensors can be buried inside concrete in order to provide the required data.

Wireless embedded sensors monitoring system has many merits. Both industry and society can benefit from the development of this novel SHM technology that reduce the reliance on slow and unreliable traditional structure fatigue/damage testing methods. Moreover, SHM technologies can be incorporated by construction processes in order to inform contractors and inspectors of critical changes and/or deficiencies of concrete structures. Further, SHM technologies will also allow industry and government to use the collected data for long-term construction management and evaluation. Therefore, the development of novel SHM methods for construction and long term performance monitoring will provide significant benefits including reliability and cost effectiveness. The impact that these systems can have to society is very significant since they can provide necessary data for analysis of infrastructure's health after events such as earthquakes, floods, hurricanes, etc. Also, such systems can warn authorities if alarming data are detected indicating critical fatigue of structures, such as, hi-rises and bridges. In summary, wireless sensors embedded in concrete can make our society safer by saving lives as well as minimizing the financial impact that failed structures can have.

Wireless embedded sensor monitoring systems present a promising solution for SHM that can benefit our society and improve the construction industry. However, the powering of wireless concrete sensors, which must perform their tasks over a long period of time (years), represents an important and challenging problem. This problem is caused by the limited life-time of sensor batteries. Conventional batteries have finite life-time, and their replacement is difficult or impossible when sensors are buried inside the 
concrete structures. Therefore, in order to enable longer operational life of wireless embedded sensors, novel wireless powering methods, which can charge the sensor's rechargeable batteries wirelessly, need to be developed. The problem of transmitting power wirelessly to sensors embedded in concrete is not a trivial one and should be rigorously analyzed to develop the needed technologies. This problem is critical to the performance of embedded sensors, and it is due to the large attenuation of radiofrequency waves due to high losses of concrete and reflection from the air-to-concrete interface. The solutions developed for air-to-air wireless power transmission do not have the same performance for air-to-concrete case. Therefore, the power channel for air-toconcrete transmission needs to be rigorously analyzed and characterized, in order to develop optimal and cost effective designs.

\subsection{Research Objectives and Contributions}

Rigorous analysis of wireless power transmission systems for sensors embedded in concrete will facilitate the development of novel and efficient NDT solutions in terms of cost and performance for constructions and monitoring of concrete structures. Also, such analysis will revolutionize the way wireless communication systems are applied to concrete, and it will solve practical and important problems of the concrete and construction industries. Our research will first produce the needed analysis for the development of wireless sensors embedded in concrete and then provide optimal solutions/designs for sensors, which perform SHM of concrete during the construction process and long-term monitoring of concrete structures. Specifically, this research performs the following tasks: 
(i) Develop reliable and accurate models calculating power transmission loss level for sensors embedded in plain/reinforced concrete, including the power loss occurring at the air-to-concrete interface and inside the lossy concrete media.

(ii) Perform comparative analysis of different SHM, RFID and wireless standards using our developed models. Identify frequency bands and RF polarizations of minimal attenuation based on the available data of concrete's electrical properties, as well as the reinforced bars' effects.

(iii) Design miniaturized and low cost prototypes of antennas that can be integrated with the sensing units at the sensor node level. The developed RF front-end designs will be optimized for the electrical properties of concrete including concrete's high losses and significant variations of its electrical parameters depending on the concrete type, humidity, curing stage and the reinforced bars' configurations inside concrete.

(iv) Develop optimum antenna matching circuits and rectification circuits in order to maximize the power delivered to the rechargeable batteries of the embedded sensors. The amount of energy received shall be sufficient to power the wireless sensors under certain duty time, and the recharging time needs to be minimized.

(v) Develop novel wireless energy harvesting techniques for sensors embedded in concrete. Perform spectrum analysis for identifying the optimum frequency range for harvesting of electromagnetic waves with least power loss inside concrete. Enable charging sensors by ambient RF waves, thus decrease the restriction of providing fixed power source. 
Our work is shown to exhibit larger wireless power transmission efficiency for sensors embedded in concrete structures than other proposed systems. Furthermore, our work is expected to have significant impact to applications that strongly rely on nondestructive testing of concrete volumetric characteristics using wireless embedded sensors. These applications include but are not limited to the following: (a) monitoring of temperature and moisture content of concrete for concrete placement and early age monitoring, (b) determining the air content after paving, and (c) collecting real-time data, such as, temperature and moisture through and beneath the plain/reinforced concrete slab, traffic loads on concrete pavements and displacement, strain, stress, etc. Such monitoring systems can be applied to various concrete structures, such as, motorways/roads, airstrips, bridges/overpasses, concrete walls, poles, dams, parking structures and footing for gates. Such systems will improve the quality of structure by enhancing the construction processes, and will warn authorities if alarming data are detected, thereby saving lives as well as minimizing the financial impact that failed structures can have. Additionally, the outcomes of this work will enable the development of efficient sensing equipment, embedded electronics, and network designs for testing lossy and frequency dependent materials, such as, concrete, soil, gravel, water, human body, etc.

\subsection{Methodology}

This dissertation addresses the problem of optimizing wireless powering of sensors embedded in concrete structures. This work demonstrates that rigorous analysis of the air-to-concrete model is critical for identifying the optimum conditions for wireless power transmission, such as, operation frequency, antenna type, position of antenna 
inside concrete, etc. These conditions will contribute to the optimum design of wireless power transmission systems.

In this work, analytical as well as computational methods are used to identify optimal conditions for wireless powering of sensors embedded in concrete. Two types of loss mechanisms are analyzed to describe the air-to-plain concrete power loss model: (a) the transmission loss and (b) the propagation loss, which are caused by the reflected and transmitted parts of electromagnetic waves propagating from air to concrete. The accurate modeling of the electromagnetic properties of concrete is critical for formulating and analyzing the air-to-concrete power transmission. The extended Debye model is used by our work. This model is frequency dependent, and it models concrete's properties more accurately than fixed permittivity models used in previous work. Also, the Debye model used here can accurately represent the relative permittivity of concrete for six distinct humidity conditions.

When reinforced bars are added in the concrete to increase the tension of structures, the electromagnetic shielding performance of reinforced concrete is dramatically changed. Limited work has been previously done on wireless power transmission through reinforced concrete. However, reinforced bars are widely used for most concrete structures. In this research work, plane wave models are used to investigate the impact of various reinforced bars configurations (i.e., mesh period, rebar radius, rebar position inside concrete slab, rebar type, etc.) on the power transmission. Furthermore, the optimum conditions identified are validated through antenna coupling simulations.

Based on the optimum transmission conditions identified by our analysis, practical powering systems are developed. Rectenna is the fundamental component for 
receiving or harvesting wireless power inside concrete structure and converting it to DC power in order to charge the rechargeable batteries of wireless embedded sensors. Optimum stripline antennas are designed for sensors embedded in concrete. They are proven to be less sensitive to the variation of concrete humidity conditions and rebar configurations. Various rectification circuits are also compared. Matched rectification circuits are developed to maximize the RF to DC power conversion efficiency.

Our theoretical EM research is obtained by our custom Matlab programs. Also, Ansoft HFSS and Ansoft Designer are used for the EM simulation analysis. Finally, Ansoft Nexxim is used for the design of rectification circuits and simulation of the entire wireless powering systems.

\subsection{Dissertation Outline}

The following chapters are organized as follows. Chapter 2 surveys the related work. In Chapter 3 plane wave analytical models for power transmission into concrete half-space and concrete slab cases are formulated. Both transmission loss and propagation loss are considered in order to identify the optimum frequencies that exhibit the least power transmission loss. Chapter 4 describes the geometrical setup of our reinforced concrete slab model. Also, in Chapter 4, conditions for optimum power transmission through reinforced concrete are identified. Various reinforced bars configurations and concrete humidity conditions are considered. In Chapter 5, computational methods are utilized to validate the optimum frequencies identified in previous chapters. Also, in Chapter 5, complete wireless powering systems are developed 
for power transmission through plain and reinforced concrete. Chapter 6 provides the conclusions of our research and identifies future research directions. 


\section{CHAPTER 2}

\section{RELATED WORK}

This chapter provides the literature review for our research. It begins with the introduction of structural health monitoring, followed by the description of wireless sensor network technologies for SHM applications. Also, wireless powering methods such as mechanical vibration harvesting, resonant coupling, strong resonant coupling and electromagnetic radiation, are presented and compared.

\subsection{Structural Health Monitoring}

The process of implementing a damage identification strategy for infrastructure is referred to as structural heath monitoring (SHM). The SHM process involves the observation of a structure over time using periodically spaced measurements, extraction of damage-sensitive features from the measurement data and implementation of statistical analysis of the extracted features [3].

For concrete structures, various methods and systems have been applied to perform the measurements and data acquisition. In [2], active acoustic transducers are used to detect the corrosion of reinforced concrete girders, while in [4] inverse synthetic aperture radar (ISAR) method is applied. Digital image correlation (DIC) [5] process involves a digital camera with specific resolution, and the whole displacement field can be measured with one picture. Vehicle with ground penetrating radar (GPR) is applied in [6] to implement the non-destructive testing for bridge decks. Optical sensors [7-8] are widely used for monitoring reinforced concrete structures, but the cost of such monitoring systems is large. Measurements of various quantities, such as strain, 
temperature, moisture, rebar corrosion, etc., are performed by such systems. The observation and measurement is the first stage of SHM applications.

Feature extraction is the process of identifying damage and fatigue of structures after the stage of measurement and data acquisition. Data fusion and compression are usually performed during this process to present the statistical distribution of the dynamically measured data, which lead to the determination of structures' damage or fatigue. Principal component analysis (PCA) is used in [9] to reduce the amount of data collected from 39 measurement points on a bridge column. Wavelet transform as a timefrequency analysis method is used in [10-11] to detect the damage position in structural components. Feature extraction transforms the measured data into damage-related information, which could lead to the damage/fatigue group classification or regression analysis.

Statistical analysis classifies the extracted feature distributions into damaged or undamaged category. Statistical analysis is based on two classes of methods: (a) supervised learning, and (b) unsupervised learning [12]. Supervised learning includes neural network [13], genetic algorithms [14], and support vector machine [15] methods, which can be applied to damage presence detections with examples from the damaged structure. Unsupervised learning includes control chart analysis [9] and hypothesis testing [16], which can be taken to high level detection, such as damage identification and classification.

These three stages of SHM systems mentioned above enable the decision making regarding public/commercial structure operation and maintenance, which are of great importance for securing lives, protecting infrastructure investments and supporting the 
vitality of our economy [17]. Several SHM systems have been proposed, for example, SHM solutions from 72 companies are reported in [18], including fatigue life indicators, fiber optics, digital image correlation and linear potentiometer, etc. The equipment for these solutions are expensive, and they also need wires attached, which may disturb the normal operation of the structure. Therefore, novel SHM technologies, which can reduce the system cost and installation complexity, need to be developed. As mentioned in [19], wireless sensors network technology is a very promising solution.

\subsection{Wireless Sensor Network for SHM Applications}

Wireless sensor network (WSN) [20] technologies attracted significant interest in recent years, and have been deployed in various applications, including habitat monitoring [21], medical monitoring [22], battlefield surveillance, and smart environment [23], etc. The conventional applications are based on air-to-air antenna communication, and they use various frequency bands from LF $(125-134 \mathrm{KHz})$ to microwave frequencies (0.3-300 GHz). Recently, sensors have been developed to be buried inside certain media and provide valuable monitoring data. For instance, underwater sensor networks [24] have found increasing use in oil-rig maintenance, coastal surveillance systems and underwater environment observations for exploration. Also, microstrip antennas and sensors were proposed in [25] for simultaneous communication and sensing of the moisture in the soil for agriculture investigation. Furthermore, antennas implanted in human body were studied in [26], aiming at monitoring various physiological parameters.

Stimulated by the development of these technologies, WSNs for structural health monitoring applications have also been developed, and the wireless monitoring system 
for large structures can be categorized into the following two configurations [27]: (a) health monitoring system with surface mounted wireless sensors, which communicate wirelessly with the interrogators, as shown in Figure 2.1(a); and (b) structural health monitoring system with wireless embedded sensors and transmitters, as shown in Figure 2.1(b).

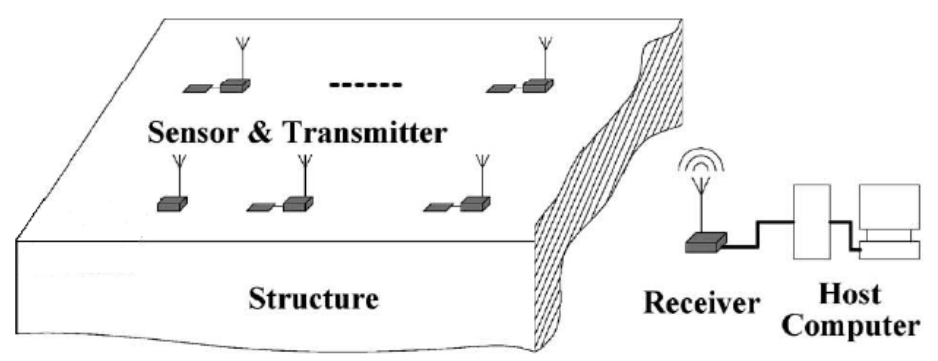

(a) Structure with surface mounted wireless sensors.

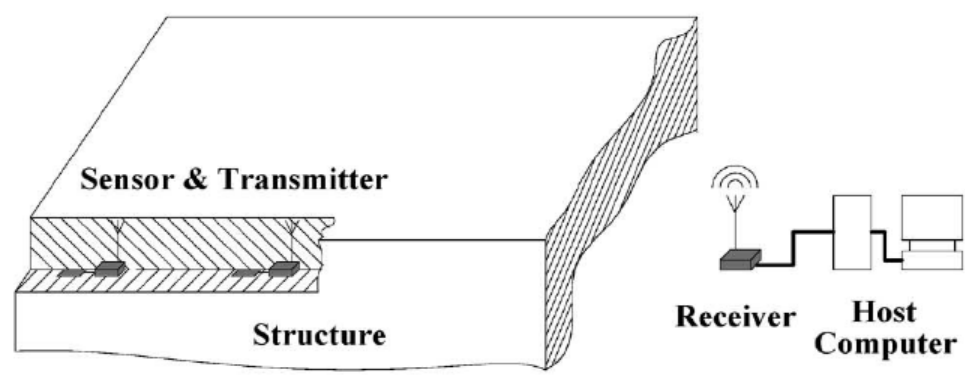

(b) Structure with wireless embedded sensors.

Figure 2.1: Configurations for sensor monitor system.

Surface mounted wireless sensor monitoring systems have been applied to structures for experiments and research in the recent years. For instance, 25 sensors are placed on three floors of a medium-sized office building to perform SHM in [28]. Another example of an SHM monitoring system, is the one deployed in Stonecutters Bridge in Hong Kong, that consist of 1505 sensors including 24 anemometers, 58 fixed accelerometers, 388 temperature sensors, 678 dynamic strain gauges, 158 static strain 
gauges, 20 global positioning systems, 34 displacement transducers, 18 buffer sensors, 12 bearing sensors, 32 electromagnetic sensors, 28 barometers, rainfall gauges and hygrometers, 33 corrosion cells, and 4 weight-in-motion stations. This SHM system makes the most rigorously monitored bridge in the world [17].

Wireless embedded sensors have also been developed for SHM systems. For example, a smart brick (i.e., brick with wireless sensors embedded), was implemented in [29] to wirelessly transmit the sampled digital and analog data to a data collection receiver with a maximum range of $101 \mathrm{~m}$. Also, a wireless embeddable sensors' platform/smart aggregate (WESP/SA) is proposed in [30] to detect the corrosion of reinforced bars in concrete bridges. Furthermore, it has been shown in [7] that the development of durable, embeddable sensors has encouraged the development of corrosion monitoring systems for new and existing reinforced concrete structures.

Advantages of wireless sensor networks for SHM applications are as follows:

(i) They shorten the deployment time and reduce cost since wiring is not needed.

(ii) They are able to measure various quantities thereby enhancing the quality of data acquisition.

(iii) They can be miniaturized using compact and inexpensive sensors, such as microelectro-mechanical systems (MEMS).

(iv) They utilize sensors that can perform monitoring without disturbing the normal operation of the structures.

(v) They can use wireless embedded sensors that enable SHM during construction period, which enhances the quality of construction processes. 
(vi) They can use wireless embedded sensors that read volumetric data inside concrete thereby providing more accurate measurement data.

Therefore, wireless embedded sensors are ideally suitable for SHM [27]. In fact, WSNs are the most promising SHM systems for large structures.

\subsection{Wireless Power Transmission for Embedded SHM Sensors}

Even though WSN technologies have many advantages for various applications, challenges still exist when they are applied to SHM applications. Such challenges include real-time monitoring, time synchronization, inexpensive system, high duty cycle, high frequency high fidelity sampling, etc., [19]. Among these challenges, power supply is one of the main limitations that restrict the development of WSNs, since most of the wireless sensors are powered using small batteries, and the conventional AA batteries could supply enough power for a typical wireless sensor working for 1 to 2 years with sleeping mode and energy-aware routing algorithm applied [31]. However, wireless sensors for SHM systems need to operate for more than 10 years, and throughout the long life-time of concrete structures. Replacing the batteries of wireless sensors over such a long period is labor intensive and time consuming. Especially, replacing batteries of sensors that are buried inside concrete are very difficult if not impossible. In order to guarantee the long life-time for wireless embedded sensors, novel wireless powering methods must be developed in order to charge sensor batteries wirelessly.

Various wireless powering methods have been proposed in the past, and could be categorized into two types: (a) power scavenging from ambient energy sources, and (b) power receiving from certain power sources through wireless power transmission 
methods. For power scavenging, solar power is collected in [32] to charge a sensor node less than $16 \mathrm{~mm}^{3}$, while thermal energy is converted to electrical energy by a low power thermoelectric generator to supply sufficient power for micro sensor in [33]. Thermal gradients are converted by thermocouples to generate an electrical power of $1.5 \mu \mathrm{W}$ in [34] in order to operate a small preamplifier and sensor control system based on a temperature difference of $10{ }^{\circ} \mathrm{C}$. Human power such as heel-strike energy is converted to electrical energy through a flexible piezoelectric foil exerted on a shoe to charge the wearable microelectronic devices [35], and $2.45 \mathrm{GHz}$ radio frequency waves is harvested in [36] by a rectangular stacked patch array to charge a sensor node. Among these methods, the piezoelectric converter and the RF wave harvester are suitable for SHM embedded sensors, since the vibration caused by the loads on bridges or the ambient commercial RF waves around the commercial buildings can be harvested for charging sensors.

Wireless power transmission is another powering solution that has been widely used for charging wireless sensors. Inductive coupling is used in [37] to charge and communicate with RFID tags, while strong resonant coupling is presented in [38] to do power transmission in mid-range. Also, electromagnetic radiation is used to charge RFID tags 44 meters away from a 4W EIRP source in [39]. These methods transmit power from fixed sources through electromagnetic/magnetic channel, and utilized either near field, mid-range or far field power transmission depending on the system requirement.

The power harvesting and wireless power transmission techniques mentioned above are widely used for air-to-air wireless powering of sensors. However, for sensors embedded in concrete, the powering system includes air-to-concrete transmission paths 
and the power receivers are buried inside lossy media. Designing powering systems for embedded sensors is extremely challenging because of the environmental effects, reduced power transmission efficiency and compact size required. Due to these reasons, mechanical vibration power harvesting and wireless power transmission techniques are promising solutions for wirelessly charging of embedded sensors in concrete, and we will discuss them in detail in the following sections.

\subsubsection{Piezoelectric Converter}

Piezoelectric phenomenon was firstly discovered in 1880s, when some researchers observed that certain types of crystalline materials may generate a voltage when being compressed. This piezoelectric effect could be implemented for converting mechanical vibration or strain variation into electric energy that could be stored in supercapacitors and rechargeable batteries.

Piezoelectric converter technologies have been proposed for charging wireless SHM sensors. For example, [40] proposed a generator capable of achieving $12.5 \mathrm{~mW}$ power with $10 \mathrm{~mm}$ displacement of the moving mass, which is sufficient to charge a wireless sensor on the bridge. Results illustrate that the output of the self-powered sensors shows a clear dependency on the traffic pattern which excites the structure, as well as the sensors' deployment positions. Experiments were done on a State Route 11 bridge in Potsdam, NY, USA, sensors positioned at approximated $1 / 3$ of the span length can achieve sufficient displacement energy, however, sensors closer to the supports may rely on different mechanism since the vibrations are insufficient there. Also, three mechanisms including lead zirconate titanate (PZT) patch, cantilever beam and PZT 
stacks/slugs are described to be suitable for embedded sensor power scavenging inside bridges in [41]. PZT patches can be used to sense the strain changes in bridge girders, and the generated power level is approximately $0.5 \mu \mathrm{W} / \mathrm{cm}^{2}$. Cantilever beam devices can generate about $20 \mu \mathrm{W} / \mathrm{cm}^{2}$ power and their dimensions can be optimized in order to maximize the power generated in various vibration environments. PZT stacks and stack arrays can achieve $1 \mathrm{~mW} / \mathrm{cm}^{2}$, which have the biggest potential among the three schemes.

Systems above illustrate that SHM sensors are able to achieve energy through piezoelectric methods, which free them from the installation of fixed power sources. However, the deployment positions of the sensors are greatly restricted by the vibration level that could be achieved by the piezoelectric materials for power conversion. For example, structures such as high-rises and dams, which may have fewer vibrations than a fully loaded hanging bridge, may not be able to provide sufficient power for the monitoring sensors embedded inside them. Therefore, novel power harvesting or power transmissions solutions are still needed for embedded SHM sensors.

\subsubsection{Inductive Coupling}

Inductive coupling is a power transmission method that has been widely used in medical [42] and industrial applications [43] for years. The phenomenon of current flow through one conductor induces a voltage across the other through electromagnetic induction is referred to as inductive coupling. Inductive coupling is applied in [44] to charge the SHM sensors embedded in concrete: the reader coil is connected to a power source outside of the concrete, and it is used for both powering and interrogating the sensor through the magnetic coupling between the reader coil and receiving coils, which 
are buried inside concrete. Similar design is also proposed in [30], where a power pickup coil working at $1 \mathrm{MHz}$ was connected to a voltage doubler for charging the bridge corrosion detection sensor, which is attached to the cross reinforced bars inside the bridge deck. In [45], a novel WSN architecture for SHM is presented, and the system is based on the adoption of contactless sensors using resonant coupling to both sense the displacements and deploy local communication network. For inductive coupling systems, power transmission and wireless communication can be performed simultaneously.

Inductive coupling for wireless power transmission was deduced due to the perception that powering or communicating though magnetic coupling does not suffer from high losses. However, there are no reliable quantitative results available regarding levels of the losses. For example, the effects of eddy currents, which are induced in the concrete and can lead to attenuation of the delivered power, have not been considered yet. Also, inductive coupling is based on near field magnetic coupling, so the distance between reader and the receiver coils are limited to only a few centimeters, which are not suitable for certain applications where larger distances are required.

\subsubsection{Strong Resonant Coupling}

Strong resonant coupling employs strongly coupled magnetic resonance to transmit power wirelessly and efficiently over a mid-range distance [46], where adverse effects of the low coupling coefficient between the two coils for inductive coupling are compensated by the high-quality factor of the four-coil system to achieve the high efficiency. It is a wireless non-radiative mid-range power transmission method that has 
been applied for power transfer in the air. This method has achieved of $40 \%$ in [46] and $60 \%$ in [47] at a distance of $2 \mathrm{~m}$.

Strongly coupled magnetic resonance (SCMR) method is proposed in [48] for powering sensors embedded in concrete. Power transmission efficiency of $60 \%$ is achieved when the coils in air are $10 \mathrm{~cm}$ from the air-to-concrete interface and the ones inside concrete are within a $7.5 \mathrm{~cm} \times 7.5 \mathrm{~cm}$ air-box at a $10 \mathrm{~cm}$ depth. Results show that high efficiency can be achieved with SCMR system when powering sensors embedded in concrete, but the size of the air-box needs to be reduced, since such large air-gap inside concrete structure may reduce the strength of it. Also, since the coils are strongly coupled in order to achieve high $Q$ at fixed frequencies, the bandwidth of the system is small. Therefore, small changes in setup of this system may dramatically impact the resonance and significantly reduce its efficiency.

\subsubsection{Electromagnetic Radiation}

Electromagnetic radiation is a form of energy exhibiting wave-like behavior as it travels through space. EM power transmission can be implemented using antenna coupling in the far field. It has been widely used in modern wireless communication systems such as GSM, GPS and RFID systems, etc.

The RF wireless power transmission system for SHM embedded sensors was deployed in field experiments on the Alamosa Canyon Bridge in 2007 [49], and the sensor node could be charged to $3.6 \mathrm{~V}$ in $27 \mathrm{~s}$ when the power source is $1 \mathrm{~W}$ and $1.2 \mathrm{~m}$ away. While in [50], a $14.5 \mathrm{dBi}$ Yagi antenna transmits $1 \mathrm{~W}$ power at $2.5 \mathrm{GHz}$ to a 19 $\mathrm{dBi}$ patch antenna $2 \mathrm{~m}$ away from it, in order to power the sensors mounted on the 
surface of Alamosa Canyon Bridge. The typical time for the $0.1 \mathrm{~F}$ capacitor to be charged to $3.5 \mathrm{~V}$ is $95 \mathrm{~s}$. A stacked patch rectenna with $75.8 \% \mathrm{DC}$ to AC conversion efficiency was designed for operation at $5.7 \mathrm{GHz}$ for wireless power transmission to a buried sensor in concrete [51]. Dry and wet concrete samples with various cover thicknesses and airgaps are considered, and $10.37 \mathrm{~mW}$ power was received at $0.6 \mathrm{~m}$ for input power of $7 \mathrm{~W}$. A circularly polarized microstrip patch antenna is designed for wireless power transmission in [52]. It operates as a rectenna working at $5.5 \mathrm{GHz}$ for data telemetry in the 5.15-5.35 GHz WLAN band. The band harmonic filter design is also proposed in [53] for a circularly polarized rectenna. A Microstrip patch antenna working at $2.45 \mathrm{GHz}$ is buried inside concrete for wireless infrastructural health monitoring in [54], and the performance of the patch is dependent on the concrete dielectric constant and loss tangent. Also, dipole and PIFA antennas working at $915 \mathrm{MHz}$ are buried inside a concrete pier for performance comparison in [55], while reinforced bar's effects are also considered.

Though several power transmission systems have been proposed, RF power communications in the concrete suffer from high losses. This problem is inherent to all wireless communications with sensors buried inside concrete, and it stems from the high attenuation of RF signals in concrete. Also, it should be pointed out that no model describing losses in communication channels for embedded sensors have been derived. For instance, no equations that are analogous to either Friis or Radar Equation have been formulated. These equations are essential for the analysis and optimization of communication channels and wireless powering transmission in heterogeneous media.

Power harvesting and wireless power transmission for embedded SHM sensors are discussed in this section. In summary, limited work has been done on this subject 
thereby necessitating further research and development in order to meet the current requirements of concrete construction as recommended by the Federal Highway Administration FHWA [56]. The existing wireless power transmission systems encounter the following problems:

(i) Lack of physical models for the complex permittivity of concrete that can accurately describe its behavior for (a) frequencies in the RF spectrum including very low frequencies, and (b) different moisture contents, temperatures, etc.

(ii) Absence of theoretical models that can predict the attenuation in communication and powering channels through concrete.

(iii) Insufficient understanding of SHM in the context of sensors embedded in concrete and absence of comparison data of different EM radiation and RFID approaches using wireless sensors buried in concrete.

(iv) Absence of analysis considering the effects of various reinforced bars types on the wireless power transmission.

(v) Absence of optimum rectenna design for power harvesting and/or receiving in concrete.

The major technological and research gaps above need to be addressed in order to develop optimal and cost effective wireless power transmission solutions for the SHM sensors embedded in concrete.

\subsection{Summary}

In this chapter, the application background related to the wireless embedded SHM sensors has been described, followed by the comparison of various wireless power 
transmission and power harvesting methods proposed for charging embedded sensors wirelessly. It could be concluded from this chapter that although many wireless powering techniques have been presented, limited work has been done on optimizing the power transmission system and maximizing the power transmission efficiency for air-toconcrete applications. 


\section{CHAPTER 3}

\section{PLANE WAVE MODEL FOR PLAIN CONCRETE}

In this chapter, optimization of wireless powering of sensors embedded in plain concrete is investigated. Our analysis relies on plane wave propagation models. Specifically, transmission loss and propagation loss of RF waves penetrating into concrete are calculated for concrete half-space and concrete slab cases. Also, electromagnetic properties of the concrete are modeled using extended Debye model.

\subsection{Extended Debye Model}

In order to describe the losses of RF waves penetrate through concrete, electromagnetic (EM) properties (i.e., relative permittivity, conductivity, etc.) of concrete need to be accurately modeled. Several models regarding the relative permittivity and conductivity of concrete at different moisture conditions and frequencies have been reported. Specifically, dielectric constant conductivity dispersion of Portland cementbased binders was measured over the $1 \mathrm{KHz}-1 \mathrm{MHz}$ range in [57]. In [58], dielectric constant, loss factor, and the conductivity of concrete was measured and provided through $0.1-20 \mathrm{GHz}$ range with four different moisture content values considered, including air dried, oven dried, wet and saturated conditions. Furthermore, Jonscher model was used in [59] to show that concrete is a dispersive medium in the $1 \mathrm{MHz}-1$ GHz range and the validation was carried out through experimental testing. Results have shown that the complex permittivity of concrete may vary with frequency and moisture content. Therefore, frequency dependent models for the relative permittivity of concrete at different humidity conditions are more accurate for our analysis. In this research, 
frequency dependent extended Debye model [60] is used to accurately describe the EM properties of the concrete for six different humidity conditions.

The relative permittivity of concrete can be written as [60]:

$$
\varepsilon_{r}(\omega)=\varepsilon_{r}^{\prime}(\omega)-j \varepsilon_{r}^{\prime \prime}(\omega)
$$

while the real part of the relative permittivity is presented as:

$$
\varepsilon_{r}^{\prime}(\omega)=\varepsilon_{\infty}+\frac{\Delta \varepsilon}{1+\omega^{2} \tau^{2}}
$$

where $\Delta \varepsilon$ stands for the difference between the values of the real part of the relative permittivity at low and high frequencies, and $\tau$ is the relaxation time. The real part of the complex relative permittivity represents the ability of the medium to store electrical energy.

The imaginary part of the complex relative permittivity represents the energy losses due to dielectric relaxation as follows:

$$
\varepsilon_{r, e f f}^{\prime \prime}(\omega)=\frac{\omega \tau \Delta \varepsilon}{1+\omega^{2} \tau^{2}}+\frac{\sigma_{d c}}{\omega \varepsilon_{0}}=\frac{\sigma_{e f f}(\omega)}{\omega \varepsilon_{0}}
$$

where $\sigma_{d c}$ is the DC electrical conductivity of concrete and $\sigma_{\text {eff }}(\omega)$ is the effective conductivity. This model can be considered as an extension of the Debye model (extended Debye model). The Debye model parameter values are available in Table 3.1 for six different humidity conditions (from $0.2 \%$ to $12.0 \%$ ). The humidity condition describes the percentage of water by volume and it is represented here by the symbol, $h$.

Relative permittivity of concrete is plot in Figure 3.1 for three humidity conditions. Results illustrate that the permittivity value for wet concrete (i.e., $12 \%$ 
humidity) is much larger than the dry concrete (i.e., $0.2 \%$ humidity), especially at lower frequencies in the 1-400 MHz range. For example, in Figure 3.1(a), when the humidity condition of wet concrete is $12 \%$, permittivity of concrete decreases dramatically as frequency increases form $1 \mathrm{MHz}$ to $400 \mathrm{MHz}$, and remains almost constant for frequencies larger than $400 \mathrm{MHz}$. However, permittivity for concrete with $0.2 \%$ moisture content hardly changes throughout the frequency range. It is approximately 3 to $8 \mathrm{~F} / \mathrm{m}$ less than the corresponding value of the wet concrete. When a plane wave propagates from air to lossy media, the reflection and transmission coefficient, together with the corresponding average power densities, are closely related to the permittivity of the media.

Table 3.1 Extended Debye model parameter values for concrete [60].

\begin{tabular}{lllll}
\hline Moisture content & \multicolumn{1}{c}{$\varepsilon_{\text {static }}$} & \multicolumn{1}{c}{$\varepsilon_{\infty}$} & \multicolumn{1}{c}{$\tau(n s)$} & \multicolumn{1}{c}{$\sigma_{\dot{d}}\left(\Omega^{-1} m^{-1}\right)$} \\
\hline $0.2 \%$ & $4.814 \pm 0.002$ & $4.507 \pm 0.002$ & $0.82 \pm 0.01$ & $6.06 \times 10^{-4} \pm 0.06 \times 10^{4}$ \\
$2.8 \%$ & $6.75 \pm 0.03$ & $5.503 \pm 0.005$ & $2.28 \pm 0.08$ & $2.03 \times 10^{-3} \pm 0.04 \times 10^{-3}$ \\
$5.5 \%$ & $8.63 \pm 0.02$ & $6.023 \pm 0.009$ & $1.00 \pm 0.02$ & $5.15 \times 10^{-3} \pm 0.06 \times 10^{-3}$ \\
$6.2 \%$ & $9.14 \pm 0.06$ & $5.93 \pm 0.02$ & $0.80 \pm 0.03$ & $6.7 \times 10^{-3} \pm 0.3 \times 10^{-3}$ \\
$9.3 \%$ & $11.19 \pm 0.05$ & $7.20 \pm 0.02$ & $0.73 \pm 0.02$ & $23 \times 10^{-3} \pm 2 \times 10^{-3}$ \\
$12.0 \%$ & $12.84 \pm 0.03$ & $7.42 \pm 0.02$ & $0.611 \pm 0.006$ & $20.6 \times 10^{-3} \pm 0.2 \times 10^{-3}$ \\
\hline
\end{tabular}

The effective conductivity of the concrete is plotted in Figure 3.2 for three humidity conditions. It can be seen from Figure 3.2 that higher humidity conditions have larger effective conductivity value. For example, as the moisture content of concrete increases from $0.2 \%$ to $12 \%$, effective conductivity of concrete increases almost 20 times at $2 \mathrm{GHz}$. The increase of effective conductivity leads to more power losses. Also, it is shown in Figure 3.2 that effective conductivity of concrete increases with frequency. For example, the effective conductivity of $6.2 \%$ humidity concrete increases dramatically as the frequencies increases form 1-600 MHz, but remains almost constant for frequencies 
larger than $600 \mathrm{MHz}$. It could be concluded that lower frequencies in our analysis range would have less power loss and are more suitable for power transmission inside concrete, especially for wet concrete cases.

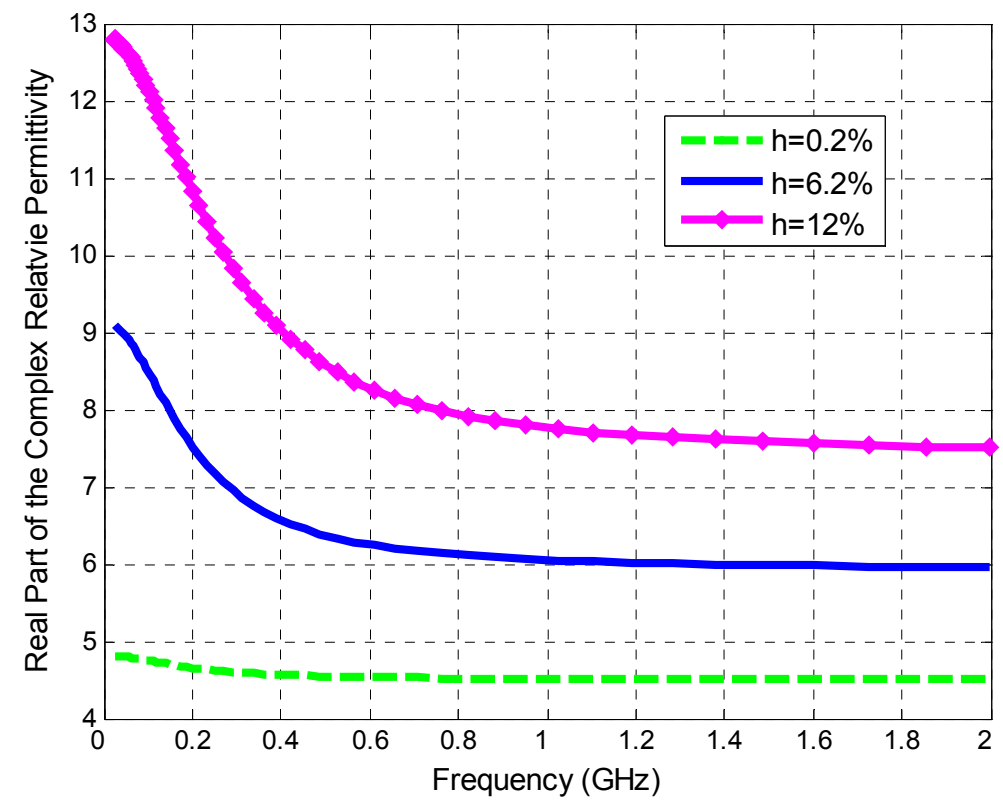

(a) Real value.

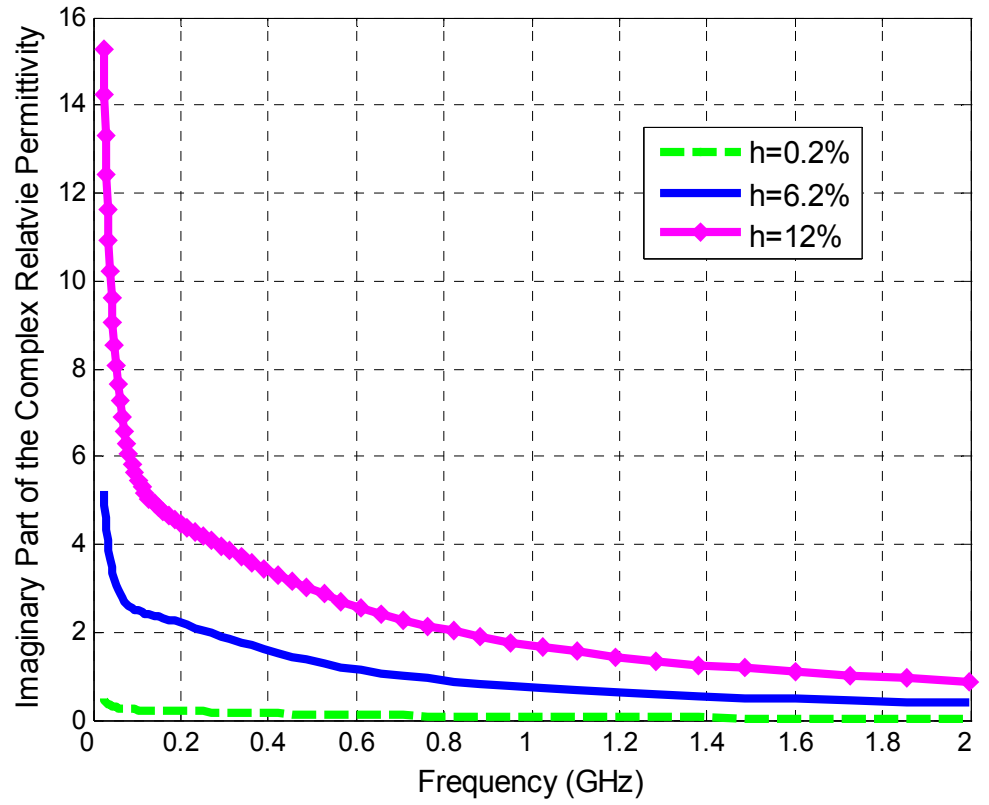

(b) Imaginary value.

Figure 3.1: Permittivity of concrete versus frequency. 
The electromagnetic properties of concrete at different humidity conditions determine the power loss level of the electromagnetic wave and the performance of the antenna buried inside it. Therefore, accurate frequency dependent permittivity models of concrete are needed for the theoretical analysis of plane wave transmission through plain concrete, which will be discussed in following sections.

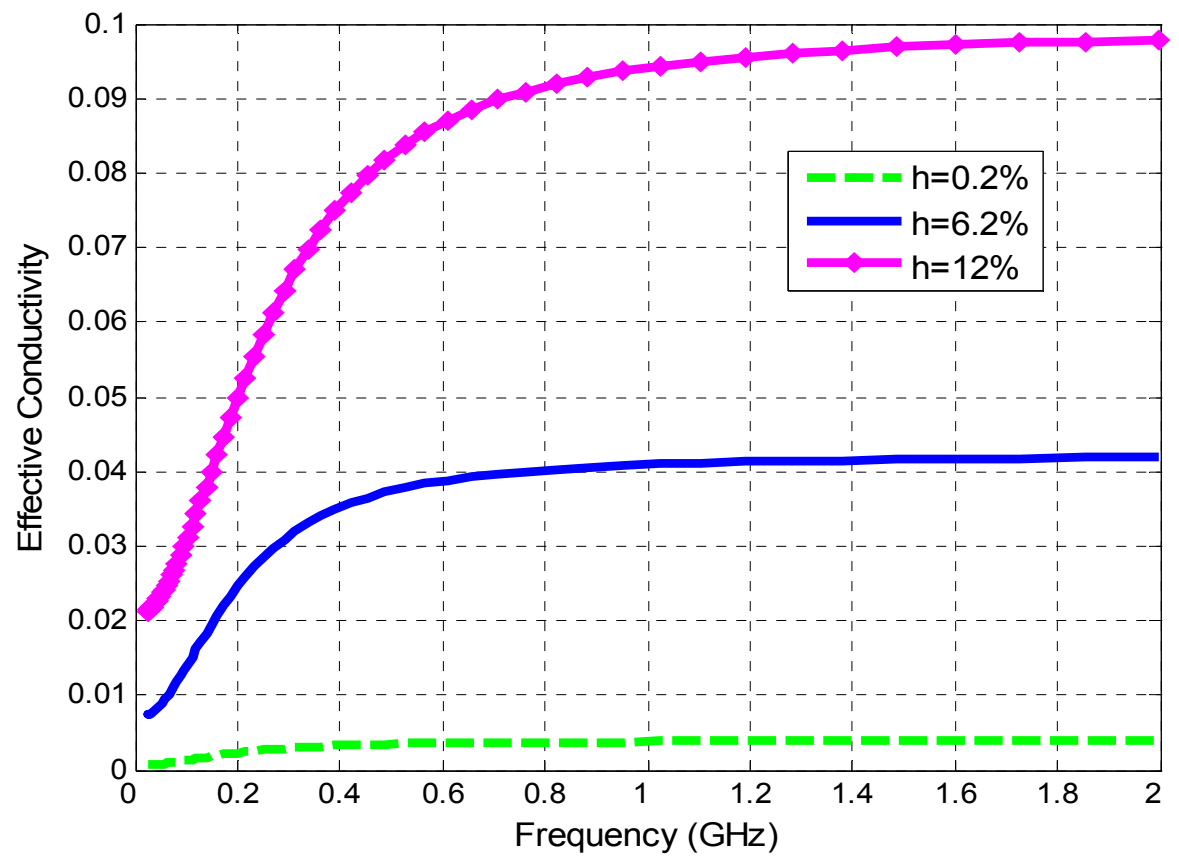

Figure 3.2: Effective conductivity of concrete versus frequency.

\subsection{Concrete Half-space Model}

For a plane wave penetrating through plain concrete, total power loss is the sum of transmission loss and propagation loss. Transmission loss is caused by the reflection of the electromagnetic wave at the air-to-concrete interface. Propagation loss is generated as the wave propagates through the lossy concrete medium. Here, we develop analytical formulations that describe both loss mechanisms and then calculate the total losses for the 
concrete half-space case. Specifically, losses of plane wave impinging on an air-toconcrete interface at normal incidence are calculated assuming the thickness of concrete is infinite, as shown in Figure 3.3.

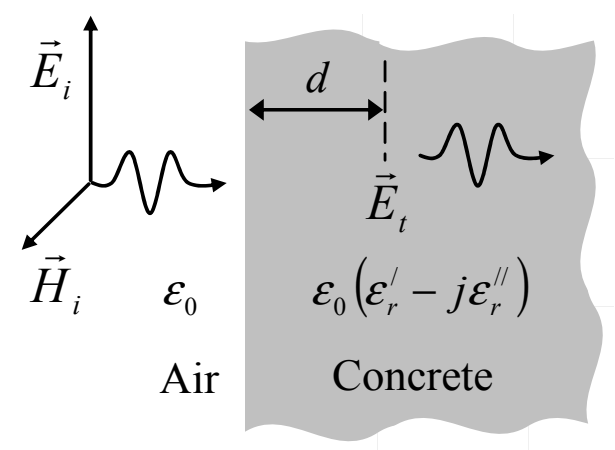

Figure 3.3: Plane wave penetrating concrete half-space at normal incidence.

\subsubsection{Normal Incidence}

First, we formulate analytical equations to calculate the transmission loss and propagation loss for the scenario of Figure 3.3 in the case of normal incidence. The incident power is written as [61]:

$$
P_{i}=\operatorname{Re}\left\{\frac{\left|E_{i}\right|^{2}}{2 \eta_{0}}\right\}
$$

where $E_{i}$ is the incident electric field and $\eta_{0}=\sqrt{\frac{\mu_{0}}{\varepsilon_{0}}}$ stands for the intrinsic impedance of air.

Similarly, the transmitted power inside concrete is written as:

$$
P_{t}=\operatorname{Re}\left\{\frac{\left|E_{t}\right|^{2}}{2 \eta_{1}^{*}}\right\}
$$


where $E_{t}$ is the transmitted electric field, $\eta_{1}=\sqrt{\frac{\mu_{1}}{\varepsilon_{0} \bar{\varepsilon}_{r}}}$ is the intrinsic impedance of concrete, and $\varepsilon_{r}=\varepsilon_{r}^{\prime}-j \varepsilon_{r}^{\prime \prime}$ stands for the relative complex permittivity of concrete [60].

Furthermore, the transferred power at a propagation depth, $d$, inside concrete can be written as:

$$
P_{t}=\operatorname{Re}\left\{\frac{\left|E_{i}\right|^{2}}{2 \eta_{1}^{*}}\right\}|\mathrm{T}|^{2} e^{-2 \alpha d}
$$

where the transmission coefficient $T$ is given by:

$$
\mathrm{T}=\frac{E_{t}}{E_{i}}=\frac{2 \eta_{1}}{\eta_{0}+\eta_{1}}
$$

It is determined by the intrinsic impedances of air and concrete, $\eta_{1}$ and $\eta_{2}$, respectively.

The attenuation constant $\alpha$ can be written as [62]:

$$
\alpha=\omega \sqrt{\mu \varepsilon}\left\{\frac{1}{2}\left[\sqrt{1+\left(\frac{\sigma}{\omega \varepsilon}\right)^{2}}-1\right]\right\}^{\frac{1}{2}}
$$

Based on (3.6), the transmission loss, which describes the power loss caused by the air-to-concrete interface, can be calculated in $\mathrm{dB}$ as follows:

$$
\alpha_{t}=10 \log _{10}\left(|T|^{2} \operatorname{Re}\left(\frac{\eta_{0}}{\eta_{1}^{*}}\right)\right)
$$

Also, the propagation loss inside concrete can be written as:

$$
\alpha_{p}=10 \log _{10}\left(e^{-2 \alpha d}\right)
$$

It increases with the propagation depth $d$.

Therefore, the total loss of the normal incidence is written as: 


$$
\begin{aligned}
\alpha_{\text {total }} & =\alpha_{t}+\alpha_{p} \\
& =10 \log _{10}\left(\frac{P_{t}}{P_{i}}\right)
\end{aligned}
$$

and it depends on the complex permittivity of concrete and the depth of propagation.

Equations (3.6)-(3.11) are used to calculate the transmission, propagation and total losses for a plane wave traveling from air to a concrete half-space in the frequency range of $1 \mathrm{MHz}-1 \mathrm{GHz}$ and for three humidity conditions. Transmission losses are plotted in Figure 3.4. Figure 3.4 shows that transmission losses decrease rapidly as the frequency increases from $1 \mathrm{MHz}$ to $20 \mathrm{MHz}$, and then remains almost constant for frequencies larger than $20 \mathrm{MHz}$. For example, the transmission loss for $12 \%$ humidity at $1 \mathrm{MHz}$ is about $8 \mathrm{~dB}$ larger than the one at $1 \mathrm{GHz}$. This is expected since the transmission loss increases as the relative permittivity of the concrete increases. As shown in Figure 3.1, the relative permittivity of concrete is significantly larger in the $1 \mathrm{MHz}$ to $400 \mathrm{MHz}$ range than the one at larger frequencies, which leads to larger transmission losses. Therefore, in order to decrease the transmission loss of plane waves travelling from air to concrete, frequencies larger than $20 \mathrm{MHz}$ should be used.

Our results also illustrate that transmission losses increase as the humidity of concrete increases. For example, at $1 \mathrm{MHz}$, the transmission loss for wet concrete (i.e., $12 \%$ humidity) is approximate $7 \mathrm{~dB}$ larger than the transmission loss for dry concrete (i.e., $0.2 \%$ humidity) case. This is expected, since as the moisture content increases from $0.2 \%$ to $12 \%$, the effective conductivity of concrete increases almost 20 times, as shown in Figure 3.2, thereby causing more losses. Specifically, we discuss the humidity effects because many applications require monitoring the properties of concrete while it is still 
wet and curing. For example, high strength concrete may take 6 to 60 days to cure depending on the structure and the weather conditions. Therefore, high strength concrete needs to be closely monitored by sensors while it is curing (it is wet) to ensure that it reaches its full strength before any mechanical load is applied to it. Hence, when designing wireless powering systems, the concrete's moisture content should be taken into account to maximize the RF power transfer.

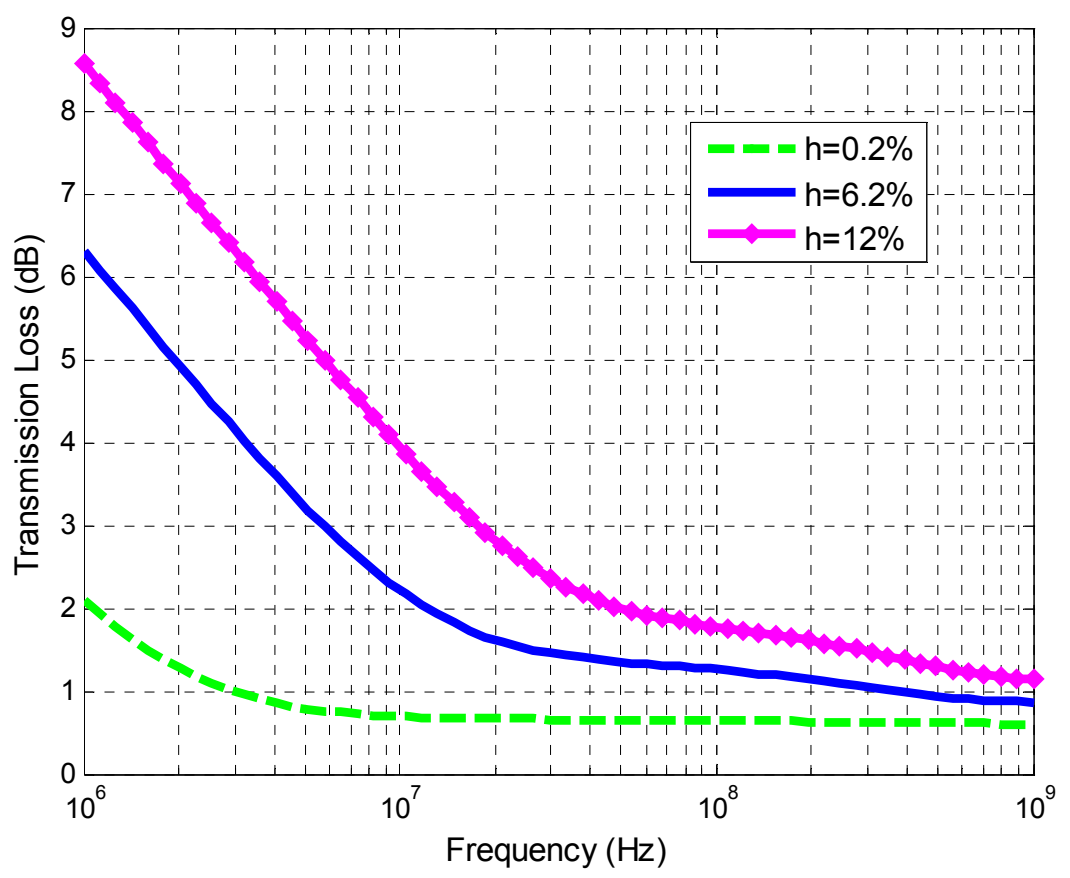

Figure 3.4: Transmission loss for normal incidence at different humidity conditions.

Then propagation loss is calculated using (3.10) and it is plotted in Figure 3.5. These results illustrate that the propagation loss exhibits an opposite trend than the one of transmission loss. For example, propagation loss for $12 \%$ humidity concrete increases slowly for frequencies less than $100 \mathrm{MHz}$ but increases dramatically for frequencies larger than that. This is expected, since propagation loss is closely related to the conductivity of the lossy media, and it increases when conductivity value increases. 
Specifically, as shown in Figure 3.2, the effective conductivity of $12 \%$ humidity concrete increases dramatically and almost keeps constant after $600 \mathrm{MHz}$, which leads to similar trend of the propagation loss. Therefore, when designing wireless powering system, frequencies smaller than $100 \mathrm{MHz}$ should be chosen for SHM sensors with medium embedded depth (i.e., 4").

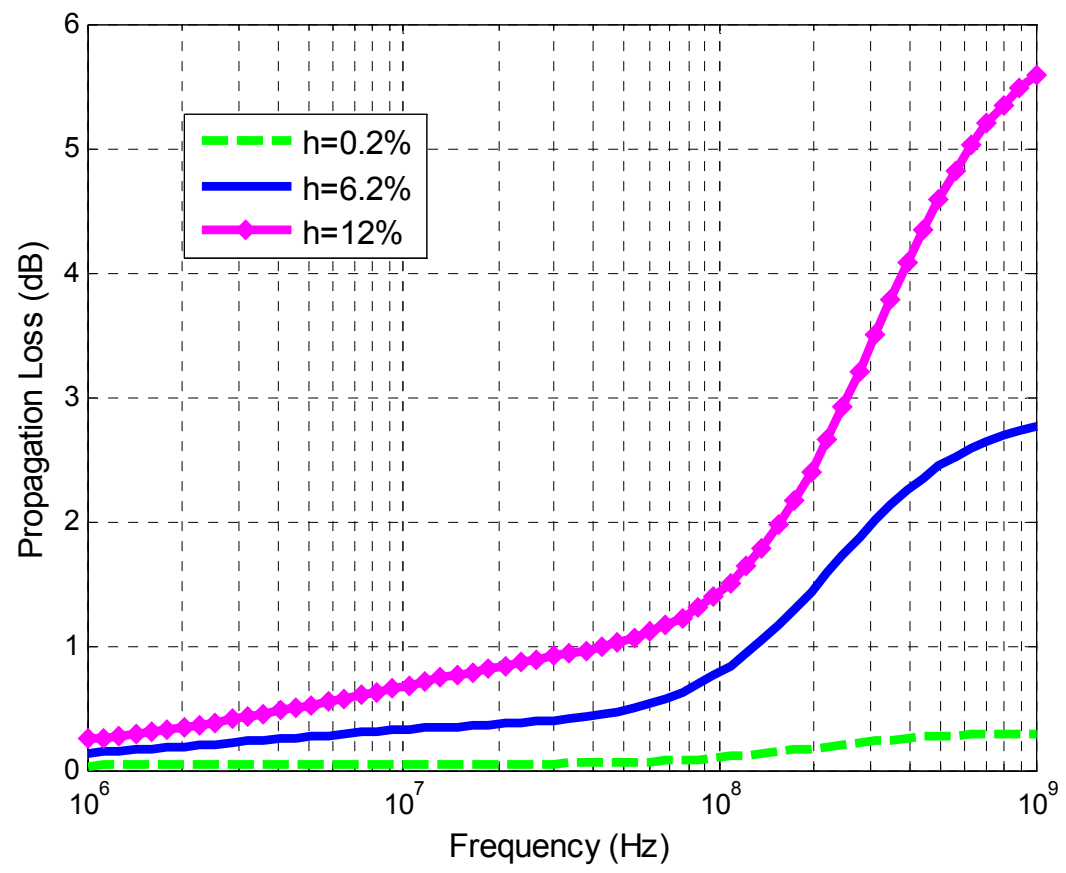

Figure 3.5: Propagation loss for normal incidence with $\mathrm{d}=10.16 \mathrm{~cm}$.

Furthermore, transmission loss and propagation loss are added to obtain the total loss for air-to-concrete propagation, as shown in Figure 3.6. As expected, due to the reverse variations of the two losses, an optimum frequency range exists, within which there is significantly smaller power loss. For example, the total loss in the frequency ranges of $20-80 \mathrm{MHz}$ for wet concrete (12\% humidity) is about 4-6 dB less than the total loss at the lowest or highest frequency in our analysis. This conclusion is particularly 
useful for powering of and communication with sensors embedded in concrete, where we seek to minimize the total power losses in order to deliver maximum power to the sensors.

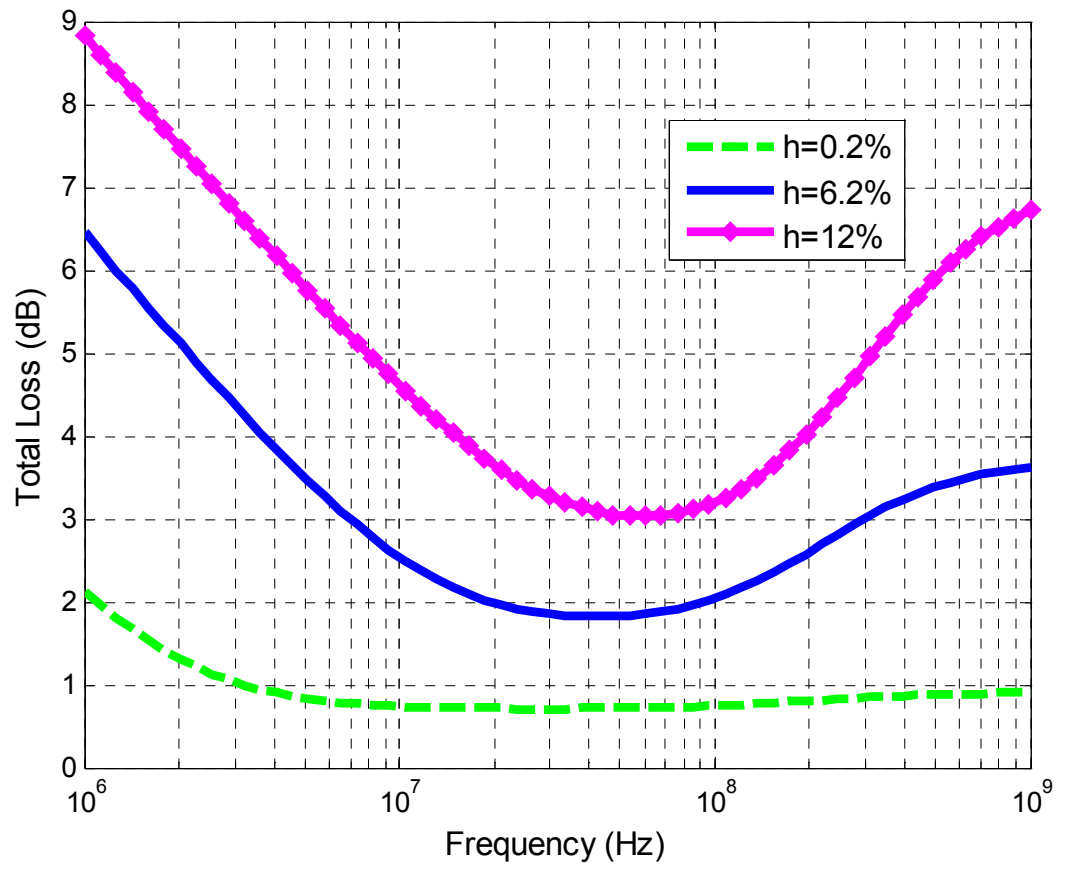

Figure 3.6: Total loss for normal incidence with $\mathrm{d}=10.16 \mathrm{~cm}$.

So far we have examined the total loss of power transmission through plain concrete half-space. Since propagation loss is closely related to the propagation depth, total loss versus frequencies and propagation depth is also examined and shown in Figure 3.7. Figure 3.7 illustrates the changing of optimum frequency range versus propagation depth $(0-25 \mathrm{~cm})$ for three humidity conditions. $0-25 \mathrm{~cm}$ is chosen since sensors may be buried in different depths inside the concrete to fulfill different application requirements in this range. Results show that when the propagation depth, $d$, is zero, total loss contains only the transmission loss, and frequencies larger than $20 \mathrm{MHz}$ suffer from less power loss, as shown in Figure 3.4. As propagation depth increases, the propagation loss also increases and the transmission loss remains the same. These trends provide a total loss 
that is optimum in a frequency range. This optimum frequency range shifts gradually to lower frequencies and falls in the $20-80 \mathrm{MHz}$ band as the propagation depth increases. For example, the total loss within the $20-80 \mathrm{MHz}$ frequency range is approximately $4-10$ $\mathrm{dB}$ less than the lowest and highest frequencies in our analysis when the propagation depth is $25 \mathrm{~cm}$ inside $12 \%$ humidity concrete.

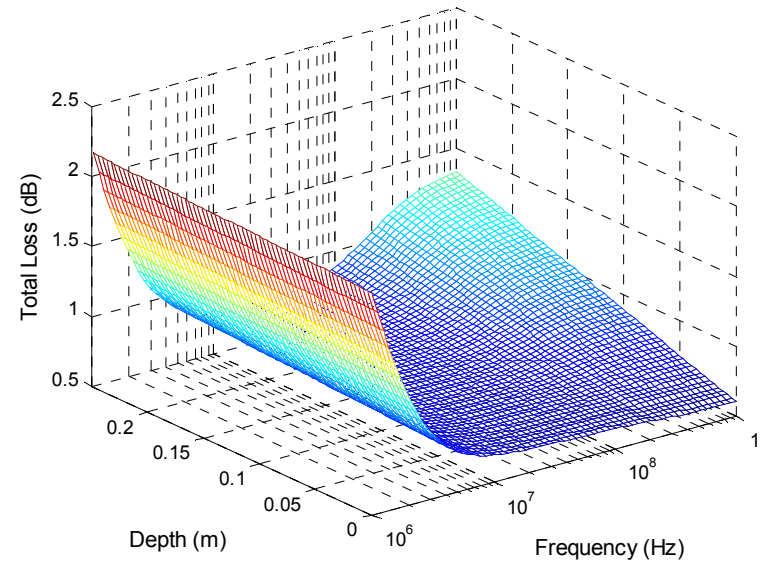

(a) $h=0.2 \%$.

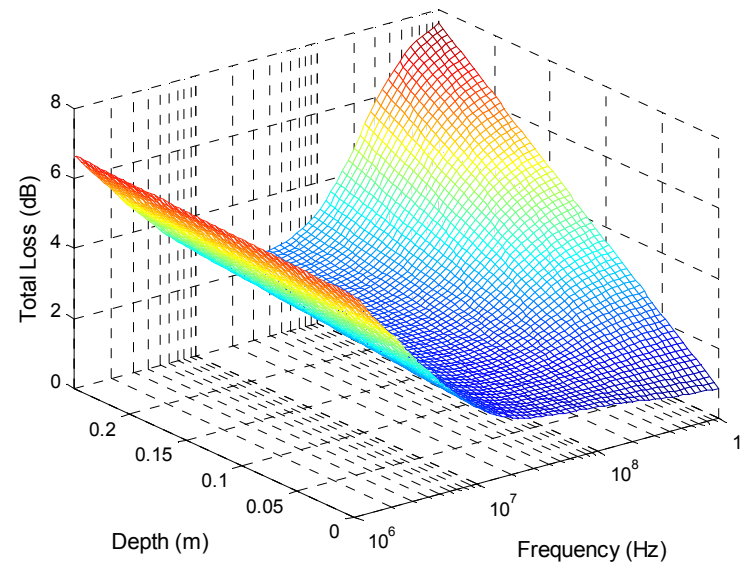

(b) $h=6.2 \%$.

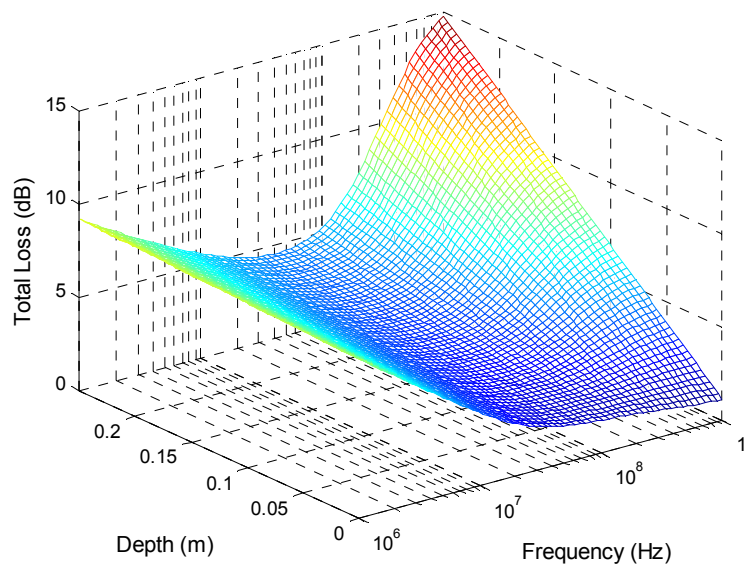

(c) $h=12 \%$.

Figure 3.7: Total loss versus frequency and propagation depth. 
Also, results illustrate that the optimum frequency range shifts faster for wet concrete (i.e., $12 \%$ humidity) than dry concrete (i.e., $0.2 \%$ humidity). For example, the difference of the total losses for $0 \mathrm{~cm}$ and $25 \mathrm{~cm}$ propagation depths at $1 \mathrm{GHz}$ for wet concrete is approximately $13 \mathrm{~dB}$, but the corresponding difference for dry concrete is only about $1 \mathrm{~dB}$. This is expected, as the effective conductivity of wet concrete is much larger (about 20 times) than dry concrete, which leads to larger propagation loss as the propagation depth increases. It can be concluded that for sensors embedded in concrete at medium depth, frequency range of 20-80 MHz provides significantly smaller power loss than all other frequencies in the range of $1 \mathrm{MHz}$ to $1 \mathrm{GHz}$. Therefore, this frequency range can be used to increase power transmission efficiency and reduce the battery charging time of embedded sensors.

So far, we have identified an optimum frequency range within $20-80 \mathrm{MHz}$ for wireless power transmission through concrete half-space at normal incidence. It should be pointed out that this frequency range includes the bands of shortwave radio (3-30 MHz) and VHF TV (54-72 MHz and 76-88 MHz). Therefore, sensors embedded in concrete operating in the optimum frequency range will also be able to efficiently harvest existing $\mathrm{RF}$ signals in these bands for charging.

\subsubsection{Oblique Incidence}

We examined the power transmission for normal incidence in the previous section. However, in practice electromagnetic waves may be incident into the concrete at an oblique angle due to the position of the power source. In order to examine the power loss at oblique incidence for a general wave polarization, it is convenient to decompose the 
electric field into perpendicular and parallel components and analyze them separately. The total reflected and transmitted field would be the vector sum from both polarizations [62]. Figure 3.8 shows the two polarizations for a plane wave incident into concrete halfspace. When the electric field is parallel to the plane of incidence, as shown in Figure 3.8(a), the polarization of the wave is referred to as parallel polarization, which is also called vertical or $\mathrm{H}$ polarization since the electric field is perpendicular to the interface and the magnetic field is parallel to the interface. When the electric field is perpendicular to the plane of incidence, as shown in Figure 3.8(b), the polarization of the wave is referred to as perpendicular polarization, which is also called horizontal or E polarization, since the electric field is parallel to the interface. The analysis of power transmission for perpendicular and parallel polarizations will enable the calculation of total power loss at oblique incidence.

As shown in Figure 3.8, plane wave penetrates through the concrete at an angle of incidence $\theta_{i}$ for both parallel and perpendicular polarizations. Due to the refraction of the wave at the air-to-concrete interface, the wave propagations into concrete half-space at an angle of $\theta_{t}$. This angle of transmission is given by Snell's law of refraction [62] as:

$$
\cos \theta_{t}=\sqrt{1-\sin ^{2} \theta_{i} / \varepsilon_{r}}
$$

As illustrated previously by (3.7), the transmission coefficient $T$ depends on intrinsic impedances of the air and concrete for normal incidence. For oblique incidence, the angle of incidence and transmission should be used for deduction of the electric field's normal components. Therefore, the transmission coefficients for parallel and perpendicular polarization are presented by: 


$$
\begin{gathered}
\mathrm{T}_{/ /}=\frac{2 \eta_{1} \cos \theta_{i}}{\eta_{0} \cos \theta_{i}+\eta_{1} \cos \theta_{t}} \\
\mathrm{~T}_{\perp}=\frac{2 \eta_{1} \cos \theta_{i}}{\eta_{1} \cos \theta_{i}+\eta_{0} \cos \theta_{t}}
\end{gathered}
$$

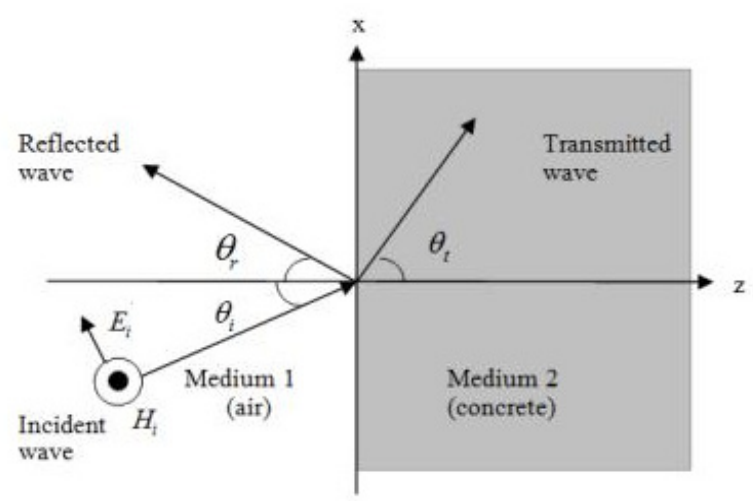

(a) Parallel polarization.

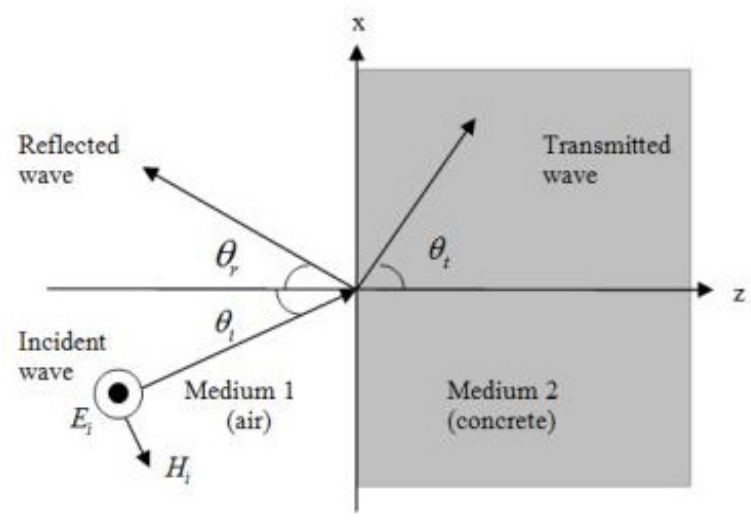

(b) Perpendicular polarization.

Figure 3.8: Plane wave penetrating concrete half-space at oblique incidence.

In order to study dependency of the transmission coefficient on different humidity conditions of concrete, we calculate the transmission coefficients for parallel and perpendicular polarizations for a plane wave at $50 \mathrm{MHz}$. Results are presented in Figure 3.9 for incidence angles ranging from 0 to 89 degrees (normal incidence corresponds to 0 degree). It is shown in Figure 3.9 that the transmission coefficient for dry concrete (i.e., 
$0.2 \%$ humidity) is larger than the one for wet concrete (i.e., $12 \%$ humidity) through all incidence angles for both polarizations. This is expected since the transmission coefficient increases as the relative permittivity of the lossy media decreases.

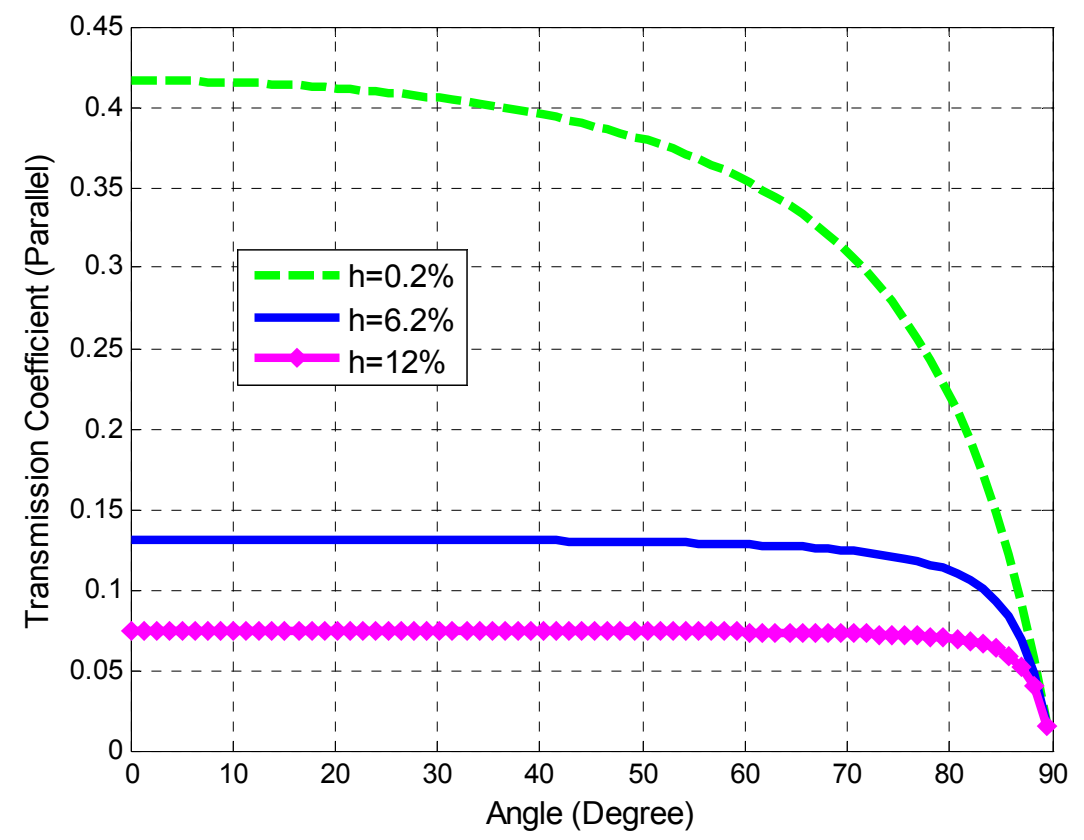

(a) Parallel polarization.

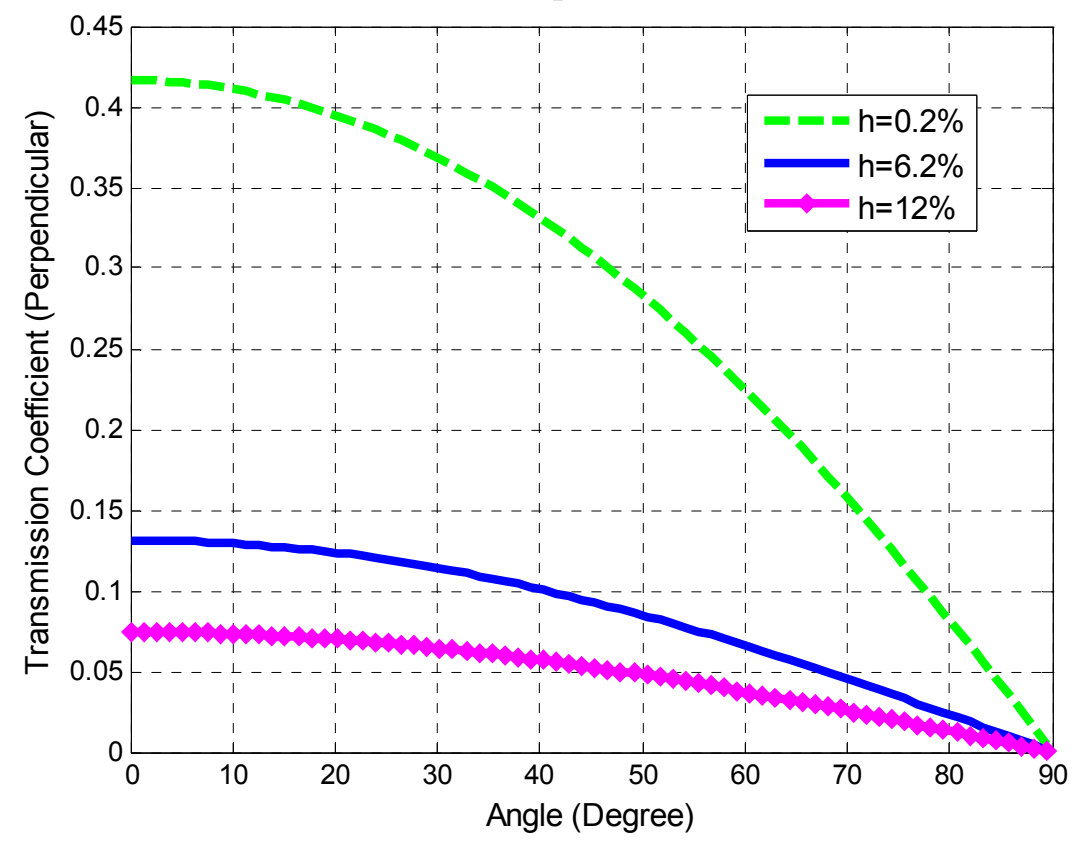

(b) Perpendicular polarization.

Figure 3.9: Transmission coefficient for oblique incidence at $50 \mathrm{MHz}$. 
Figure 3.9 also illustrates that transmission coefficient decreases as the incidence angle increases in both parallel and perpendicular polarizations. In fact the transmission coefficient for perpendicular polarization decreases more rapidly than the one for parallel polarization as the incidence angle increases. For example, the transmission coefficient for parallel polarization at $6.2 \%$ humidity remains almost constant up to angle of incidence of 80 degrees and then rapidly decreases for larger angles of incidence [see Figure 3.9(a)]. However, as illustrated in Figure 3.9(b), the transmission coefficient for perpendicular polarization gradually decreases as the angle of incidence increases from 0 to 90 degrees. It can be concluded that larger transmission coefficient is achieved at normal incidence. Furthermore, for oblique incidence cases, parallel polarization provides larger power transmission through concrete. When designing wireless powering systems for sensors embedded in concrete, the angle of incidence and polarizations must be carefully considered as they can significantly affect the performance of system.

The power loss for plane wave propagation into concrete half-space in oblique incidence cases can be calculated based on the transmission coefficient values. The transmission loss for oblique incidence can be calculated in similar fashion with (3.9):

$$
\begin{aligned}
& \alpha_{t / /}=10 \log _{10}\left(\left|\mathrm{~T}_{/ /}\right|^{2} \operatorname{Re}\left(\frac{\eta_{0}}{\eta_{1}^{*}}\right)\right) \\
& \alpha_{t \perp}=10 \log _{10}\left(\left|\mathrm{~T}_{\perp}\right|^{2} \operatorname{Re}\left(\frac{\eta_{0}}{\eta_{1}^{*}}\right)\right)
\end{aligned}
$$

It is seen that the power loss depends on the transmission coefficients for parallel and perpendicular polarizations as well as the intrinsic impedances of air and concrete.

The propagation loss for oblique incidence can be written as follows: 


$$
\alpha_{p / /}=\alpha_{p \perp}=10 \log _{10}\left(e^{-2 \alpha d / \cos \left(\theta_{t}\right)}\right)
$$

It is seen that the wave propagation distance to certain propagation depth inside concrete is increased by the factor which depends on the transmission angle.

Accordingly, the total loss for the oblique incidence can be written as:

$$
\begin{gathered}
\alpha_{t o t a l / /}=\alpha_{t / /}+\alpha_{p / /} \\
\alpha_{t o t a l \perp}=\alpha_{t \perp}+\alpha_{p \perp}
\end{gathered}
$$

Following similar steps as the ones for normal incidence case, the average total loss across all incidence angles ranging from 0 to 89 degrees is calculated for frequencies from $1 \mathrm{MHz}$ to $1 \mathrm{GHz}$ (normal incidence corresponds to 0 degree). Figure 3.10 plots the average total loss versus frequency for both polarizations. Based on Figure 3.10, it can be concluded that the average total loss for an oblique incident plane wave is larger than the normal incidence case (see Figure 3.6), as expected, but both cases exhibit similar variation. For example, the average total loss for perpendicular polarization in the $20-80$ $\mathrm{MHz}$ frequency range is approximately $5 \mathrm{~dB}$ larger than the total loss for normal incidence in the case of $12 \%$ humidity [see Figure 3.6 and 3.10(b)]. Therefore, when a plane wave is normally incident at a frequency in the range $20-80 \mathrm{MHz}$, an embedded sensor will receive significantly larger power than the other angles and frequencies. Also, for the case of oblique incidence we see that the optimum frequency range still matches the optimum range that we identified for the normal incidence (20-80 MHz). 


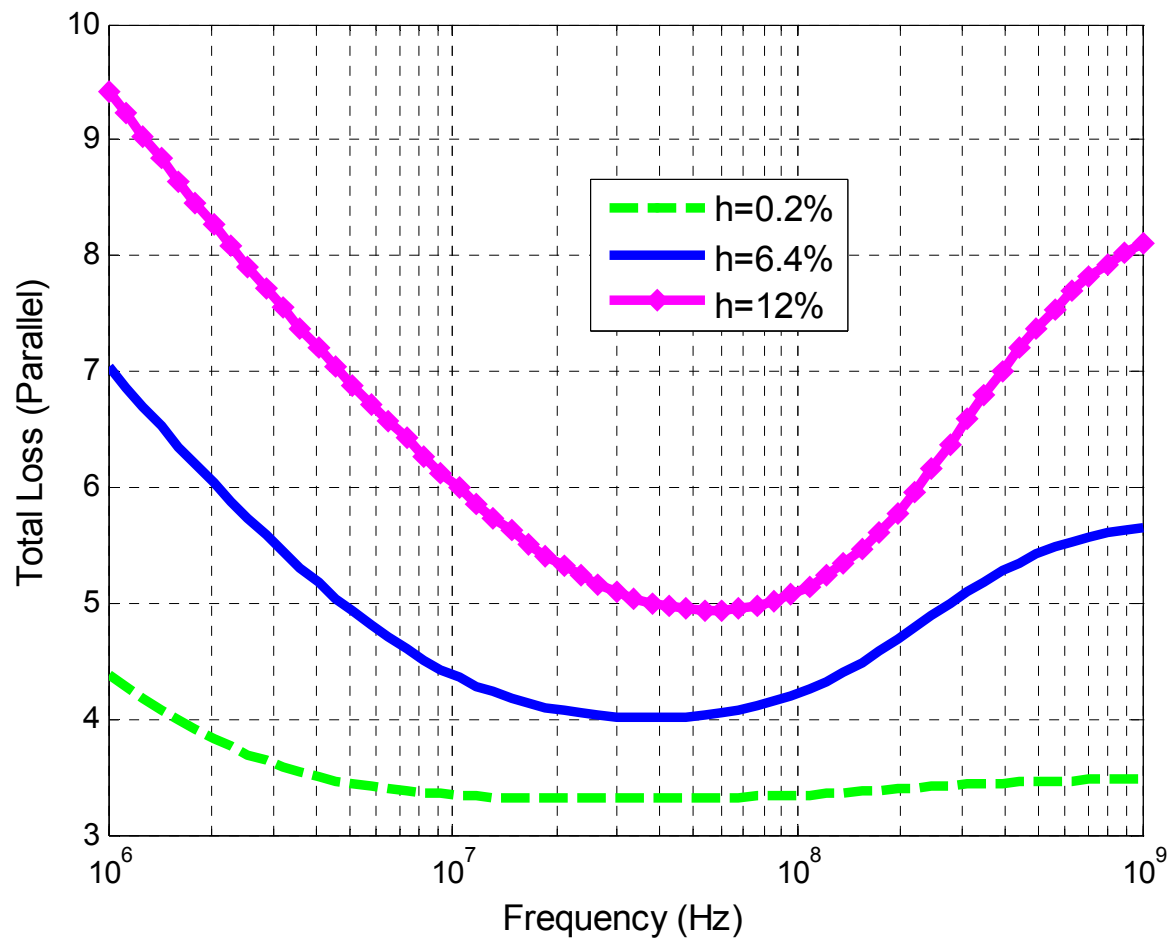

(a) Parallel polarization.

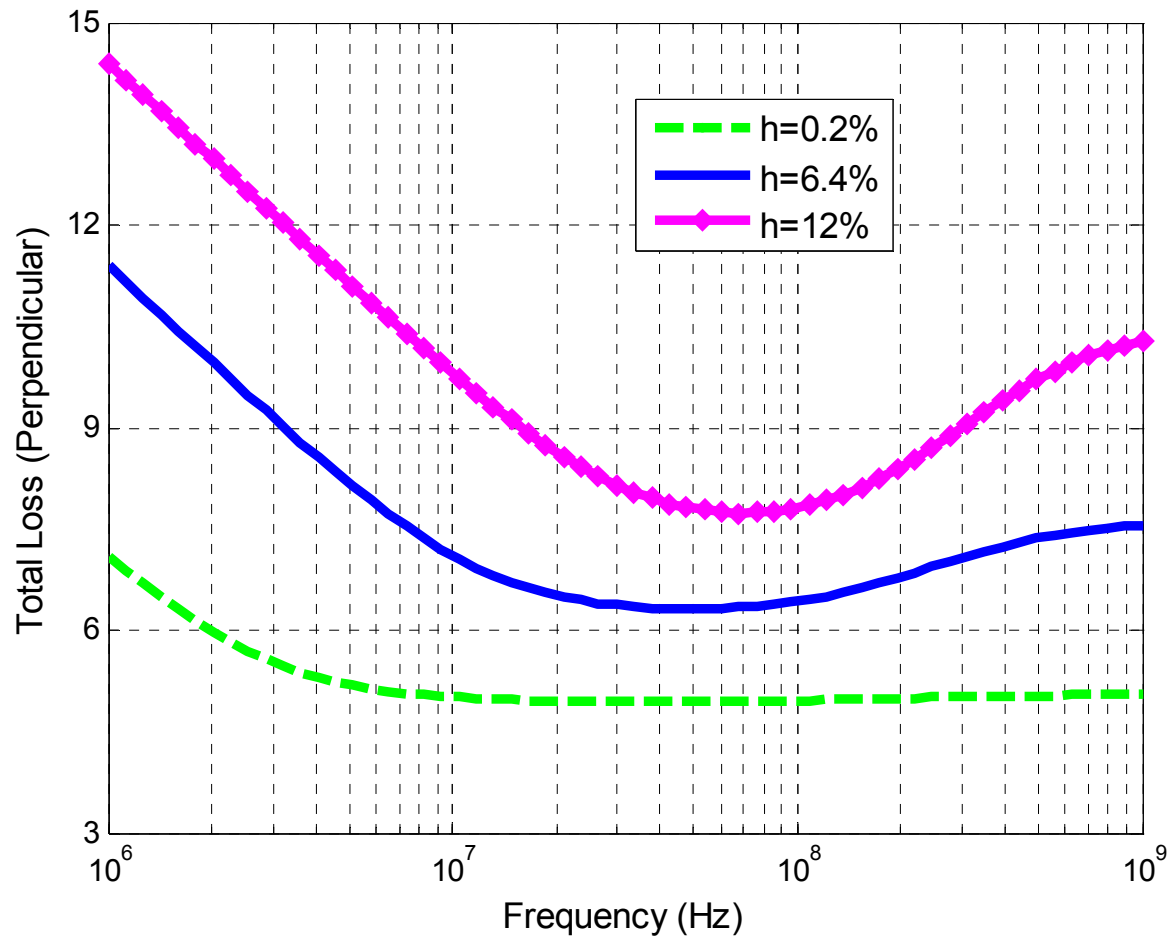

(b) Perpendicular polarization.

Figure 3.10 Total loss for oblique incidence at a depth $\mathrm{d}=10.16 \mathrm{~cm}$. 


\subsection{Concrete Slab Model}

In the previous section, we examined wireless power transmission through concrete half-space and identified an optimum frequency range of $20-80 \mathrm{MHz}$ that provides minimum power losses. In this section, power transmission through concrete slabs (with finite thickness) is investigated. Concrete slabs are typical building materials which are widely used for construction, i.e., concrete brick, footing block, etc. Therefore, studying power loss for concrete slab may contribute to the development of optimum powering system for SHM sensors that are embedded inside them.

The air-to-concrete slab model is shown in Figure 3.11, where the plane wave is impinging on the concrete slab at normal incidence assuming that the thickness of concrete is finite. Figure 3.11 also illustrates that the electric field induced at certain depth $d_{l}$ inside the concrete combines the electric field of the transmitted wave (from airto-concrete interface) and the reflected wave (from the concrete-to-air interface). The transmission and reflection coefficients for a plane wave incident on a concrete slab are shown in Figure 3.12.

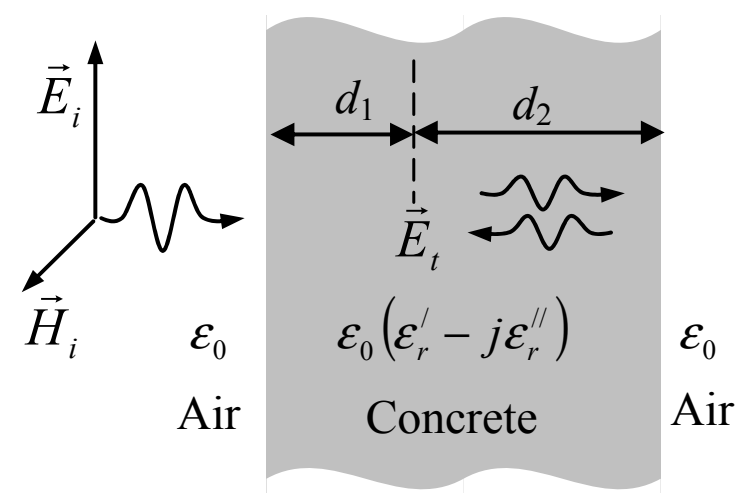

Figure 3.11: Plane wave penetrating concrete slab at normal incidence. 


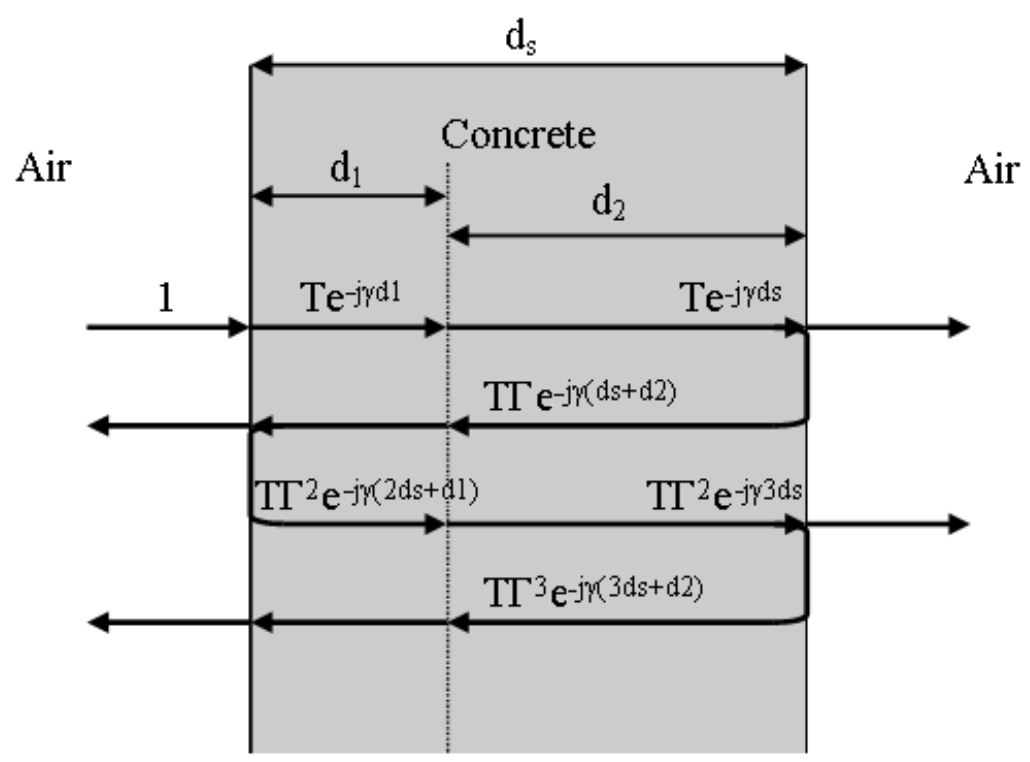

Figure 3.12: Transmission and reflection coefficients for plane wave penetrating a concrete slab at normal incidence.

It is illustrated in Figure 3.12 that at $d_{1}$ depth inside the concrete slab, the initial electric field is the sum of two different direction components $\mathrm{T} e^{-j \gamma d_{1}}$ and $\mathrm{T} \Gamma e^{-j \gamma\left(d_{s}+d_{2}\right)}$, where $T$ and $\Gamma$ stand for the transmission coefficient and reflection coefficient, respectively, and $\gamma$ is the complex propagation constant. Since plane wave may be reflected to and fro inside the concrete slab at the air-to-concrete and concrete-to-air interfaces, reflected waves would be decreased with the common ratio of $\Gamma^{2} e^{-j \gamma 2 d_{s}}$, which includes the reflection coefficient and the total thickness of the concrete slab. Therefore, the electric field can be expressed as the geometric series. The summation of geometric series with initial value $a$ and common ratio $r$ is given by:

$$
\begin{aligned}
\sum_{k=0}^{n} a r^{k} & =a r^{0}+a r^{1}+a r^{2}+\cdots+a r^{n} \\
& =\frac{a-a r^{n+1}}{1-r}
\end{aligned}
$$


when $r<1, n \rightarrow \infty$, the summation could be calculated as:

$$
\sum_{k=0}^{\infty} a r^{k}=\frac{a-a r^{\infty}}{1-r}=\frac{a}{1-r}
$$

When calculating the electric field at depth $d_{l}$ inside the concrete slab, the corresponding common ratio $r$ is $\Gamma^{2} e^{-j 2 \gamma d_{s}}$, which is smaller than 1 and fits the requirement of (3.21). The corresponding initial value $a$ is $\mathrm{T} e^{-j \gamma d_{1}}+\mathrm{T} e^{-j \gamma d_{s}} \Gamma e^{-j \gamma d_{2}}$ (see Figure 3.12). Therefore, the electric field at depth $d_{l}$ can be written as:

$$
E_{l}=\frac{\mathrm{T} e^{-j \gamma d_{1}}+\mathrm{T} e^{-j \gamma d_{s}} \Gamma e^{-j \gamma d_{2}}}{1-\Gamma^{2} e^{-j \gamma 2 d_{s}}}
$$

where the reflection and transmission coefficients, $\Gamma$ and $T$, respectively, depend on the permittivity of concrete, and can be expressed in terms of intrinsic impedance of air and concrete as follows:

$$
\begin{gathered}
\Gamma=\frac{\eta_{0}-\eta_{1}}{\eta_{0}+\eta_{1}}=\frac{\sqrt{\frac{\mu_{0}}{\varepsilon_{0}}}-\sqrt{\frac{\mu_{0}}{\varepsilon_{0} \varepsilon_{r}}}}{\sqrt{\frac{\mu_{0}}{\varepsilon_{0}}}+\sqrt{\frac{\mu_{0}}{\varepsilon_{0} \varepsilon_{r}}}}=\frac{\sqrt{\varepsilon_{r}}-1}{\sqrt{\varepsilon_{r}}+1} \\
\mathrm{~T}=\frac{2 \eta_{1}}{\eta_{0}+\eta_{1}}=\frac{2 \sqrt{\frac{\mu_{0}}{\varepsilon_{0} \varepsilon_{r}}}}{\sqrt{\frac{\mu_{0}}{\varepsilon_{0}}}+\sqrt{\frac{\mu_{0}}{\varepsilon_{0} \varepsilon_{r}}}}=\frac{2}{\sqrt{\varepsilon_{r}}+1}
\end{gathered}
$$

$\eta_{0}$ and $\eta_{1}$ stand for the intrinsic impedances of the air and concrete, respectively, and $\varepsilon_{r}$ is the relative permittivity of the concrete.

Also, as defined in [62], $\gamma$ in (3.22) is the complex propagation constant, and is defined as: 


$$
\begin{aligned}
\gamma & =\alpha+j \beta=j \omega \sqrt{\mu \varepsilon_{0} \varepsilon} \sqrt{1-j \frac{\sigma}{\omega \varepsilon_{0} \varepsilon}} \\
& =j \omega \sqrt{\mu \varepsilon_{0}} \sqrt{\varepsilon-j \frac{\sigma}{\omega \varepsilon_{0}}}=j \omega \sqrt{\mu \varepsilon_{0} \varepsilon_{r}}
\end{aligned}
$$

where $\alpha$ is the propagation constant, $\beta$ presents the phase constant and $\varepsilon$ stands for the real permittivity value of the material.

If we define $r_{1}=-j \omega \sqrt{\mu \varepsilon_{0} \varepsilon_{r}} d_{1}$ and $r_{2}=j \omega \sqrt{\mu \varepsilon_{0} \varepsilon_{r}} d_{2},(3.22)$ can be written as:

$$
\begin{aligned}
E_{l}= & \frac{\frac{2}{\sqrt{\varepsilon_{r}}+1} e^{-j \gamma d_{1}}+\frac{\left(2 \sqrt{\varepsilon_{r}}-1\right)}{\left(\sqrt{\varepsilon_{r}}+1\right)^{2}} e^{-j \gamma d_{1}} e^{-j 2 \gamma d_{2}}}{1-\left(\frac{\sqrt{\varepsilon_{r}}-1}{\sqrt{\varepsilon_{r}}+1}\right)^{2} e^{-j 2 \gamma d_{1}} e^{-j 2 \gamma d_{2}}} \\
= & 2 \frac{\left(\sqrt{\varepsilon_{r}}+1\right) e^{-j \gamma d_{1}} e^{j 2 \gamma d_{2}}+\left(\sqrt{\varepsilon_{r}}-1\right) e^{-j \gamma d_{1}}}{\left(\sqrt{\varepsilon_{r}}+1\right)^{2} e^{j 2 \gamma d_{2}}-\left(\sqrt{\varepsilon_{r}}-1\right)^{2} e^{-j 2 \gamma d_{1}}} \\
= & 2 \frac{\left(\sqrt{\varepsilon_{r}}+1\right) r_{1} r_{2}^{2}+\left(\sqrt{\varepsilon_{r}}-1\right) r_{1}}{\left(\sqrt{\varepsilon_{r}}+1\right)^{2} r_{2}^{2}-\left(\sqrt{\varepsilon_{r}}-1\right)^{2} r_{1}^{2}}
\end{aligned}
$$

The total loss for the concrete slab scenario of Figure 3.11 can be deduced following a similar procedure to the one presented in [63] as:

$$
\alpha_{\text {total }}=10 \log _{10}\left(\left|T_{\text {slab }}\right|^{2} * \operatorname{Re}\left(\frac{\eta_{0}}{\eta_{1}^{*}}\right)\right)
$$

where

$$
\left|T_{\text {slab }}\right|^{2}=\left|\frac{E_{l}}{E_{i}}\right|^{2}=4\left|\frac{\left(\sqrt{\varepsilon_{r}}+1\right) r_{1} r_{2}^{2}+\left(\sqrt{\varepsilon_{r}}-1\right) r_{1}}{\left(\sqrt{\varepsilon_{r}}+1\right)^{2} r_{2}^{2}-\left(\sqrt{\varepsilon_{r}}-1\right)^{2} r_{1}^{2}}\right|^{2}
$$


It should be pointed out that the total loss given by (3.28) for $d_{2} \rightarrow \infty$, i.e., for a very thick concrete slab (i.e., concrete half-space), becomes equal to the total loss given by (3.11).

The total loss of plane waves penetrate through a $12 \%$ humidity concrete slab is calculated here based on the above formulations. Figure 3.13 illustrates this loss for a propagation depth of $10.16 \mathrm{~cm}$ and for three different concrete thicknesses, i.e., $2 \mathrm{~m}, 5 \mathrm{~m}$ and $10 \mathrm{~m}$. Results indicate that an optimum frequency range still exists for power transmission through concrete slab. For example, total losses in the $20-80 \mathrm{MHz}$ range are 4-7 dB smaller than the losses at the lowest or highest frequencies of our analysis when the thickness of the concrete slab is $5 \mathrm{~m}$, as shown in Figure 3.13. Therefore, working inside the optimum frequency range may lead to larger wireless power transmission efficiencies.

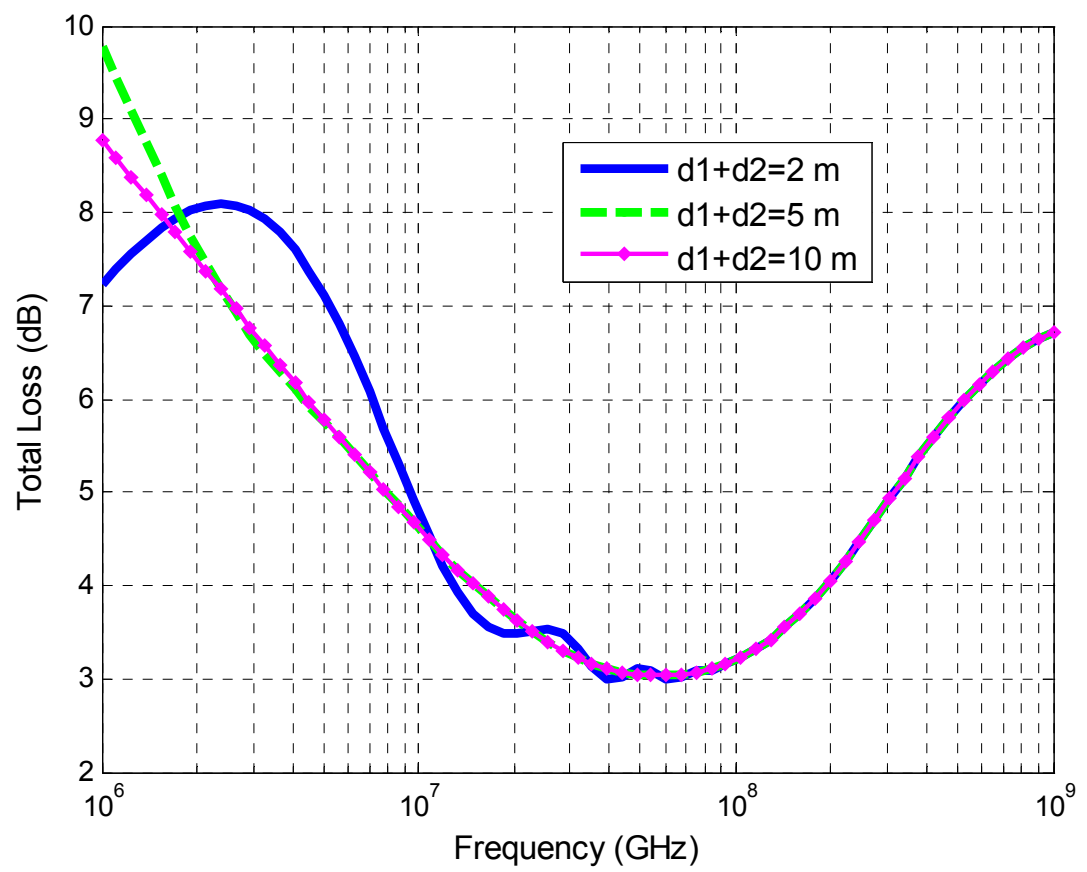

Figure 3.13: Total loss for normal incidence with $\mathrm{d} 1=10.16 \mathrm{~cm}, \mathrm{~h}=12 \%$. 
Also, the oscillatory behavior of the total loss for the case of a $2 \mathrm{~m}$ thick concrete slab (see Figure 3.13) is expected due to the superposition of primary and reflected waves. This phenomenon can be reduced as the moisture content or thickness of concrete increases. For example, as shown in Figure 3.13, when the thickness of concrete is increased to $10 \mathrm{~m}$, the corresponding total loss is approximately the same as the total loss for the concrete half-space (see Figure 3.6). However, when the concrete slab is thinner (less than half meter) and dryer, the oscillatory behavior would be more pronounced, and this will be discussed in detail in Chapter 4 .

\subsection{Model for Other Lossy Media}

In previous sections we examined the optimum frequency ranges for plane waves penetrating concrete half-space and concrete slabs. Following similar steps, optimum conditions for communication with or powering of sensors embedded in other lossy media (i.e., seawater, fresh water, soil, human-body, etc.) can be investigated. Monitoring sensors embedded in certain lossy media involves various applications. For instance, microstrip sensors were proposed in [25] for simultaneous sensing and reporting moisture contents of soil for agriculture investigation. Furthermore, antennas implanted in human body were studied in [64] and [65], aiming at monitoring of various physiological parameters. In addition, underwater sensors networks (UWSN) in [66] were developed for various applications, such as, costal surveillance, underwater environment observations for exploration, oil-fig maintenance, etc. Minimizing EM power loss is critical for embedded sensor monitoring systems. 
First, the total loss for EM waves propagating from air to fresh water is analyzed in the frequency range of $23 \mathrm{KHz}$ to $1 \mathrm{GHz}$ at normal incidence. This frequency range includes frequencies from the VLF (used in submarine communications) to the UHF band. Various propagation depths are considered since sensors or submarines may work at different depth inside fresh water.

The complex frequency dependent dielectric permittivity $\overline{\mathcal{E}}_{r}(f)$ of water is commonly described by the Debye model in [67] as:

$$
\overline{\mathcal{E}}_{r}(f)=\varepsilon_{0}+\frac{\varepsilon_{s}-\varepsilon_{\infty}}{1+\left(i \frac{f}{f_{\text {ref }}}\right)}-\frac{i \sigma}{2 \pi f \varepsilon_{0}}
$$

where $\varepsilon_{s}$ and $\varepsilon_{\infty}$ are the real relative permittivity at low and high frequencies, respectively, $f_{\text {ref }}$ is the relaxation frequency, $\sigma$ is the conductivity of water, and $\varepsilon_{0}$ is the dielectric permittivity of free space. Table 3.2 shows the Debye parameters of water reported in [67].

Table 3.2 Debye model parameter values for fresh water [67].

\begin{tabular}{lcccc}
\hline Medium & $\varepsilon_{s,[]}$ & $\varepsilon_{\infty,[]}$ & $f_{\text {ref },[\mathrm{GHz}]}$ & $\sigma, \mathrm{Sm}^{-1}$ \\
\hline Water & 80 & 4.22 & 17.4 & 0.01 \\
\hline
\end{tabular}

The total loss is calculated by substituting (3.29) into (3.11). Specifically, the total loss for plane wave propagating from air to fresh water is plotted in Figure 3.14 for shallow as well as deep propagation depths. The results of Figure 3.14(a) illustrate the existence of an optimum frequency for shallow propagation depth conditions. For example, the total loss in 3-100 MHz frequency range for shallow propagation condition ( $0.5 \mathrm{~m}$ depth) is about $10 \mathrm{~dB}$ to $30 \mathrm{~dB}$ smaller than the total loss at the lowest and highest frequencies of the analyzed frequency range. The existence of an optimum frequency 
range is particularly useful for improving RF communications with underwater vehicles or devices. Potential applications that can benefit from operating in the optimum frequency range include the following: 1) communications with underwater robots [68], 2) RFID based shore erosion detection [69], 3) water quality monitoring of bodies of fresh water (i.e., temperature, $\mathrm{pH}$, etc.), such as, artificial lakes, swimming pools and water tanks, using wireless underwater sensors.

Figure 3.14(b) also illustrates that the total loss monotonically increases when the propagation depth is larger than $10 \mathrm{~m}$, thereby providing no optimum frequency range. This happens because the transmission loss is the same for different propagation depths whereas the propagation loss increases as the propagation depth becomes larger. Therefore, as propagation depth increases it reaches a value for which the propagation loss becomes larger than the transmission loss. Therefore, the optimum frequency range exists only for small propagation depths (less than $5 \mathrm{~m}$ ).

In this optimum frequency range, the waves experience significantly smaller losses than the losses at the lowest and highest frequencies of our analysis. Specifically, this frequency range includes the bands of short-wave radio (3-30 MHz), VHF TV (54-72 MHz, 76-88 MHz), parts of FM (88-108 MHz) and US military VHF- FM band (30-88 $\mathrm{MHz}$ ). Therefore, various communications systems can benefit from using the optimum operation frequencies that we identified. Also, wireless power harvesting by wireless sensors can be significantly enhanced if it is performed inside the 3-100 MHz range.

It should also be pointed out that practical compact antenna designs can be developed in the optimum frequency range of 3-100 MHz for underwater devices due to the large permittivity of water $\left(\varepsilon_{\mathrm{r}}=81\right)$. For example, a half-wavelength loop antenna 
operating at $100 \mathrm{MHz}$ inside fresh water has a diameter of only $5.3 \mathrm{~cm}$. Therefore, the optimum frequency range can be used in practical underwater communication systems and it will provide minimum losses for shallow propagation depths.

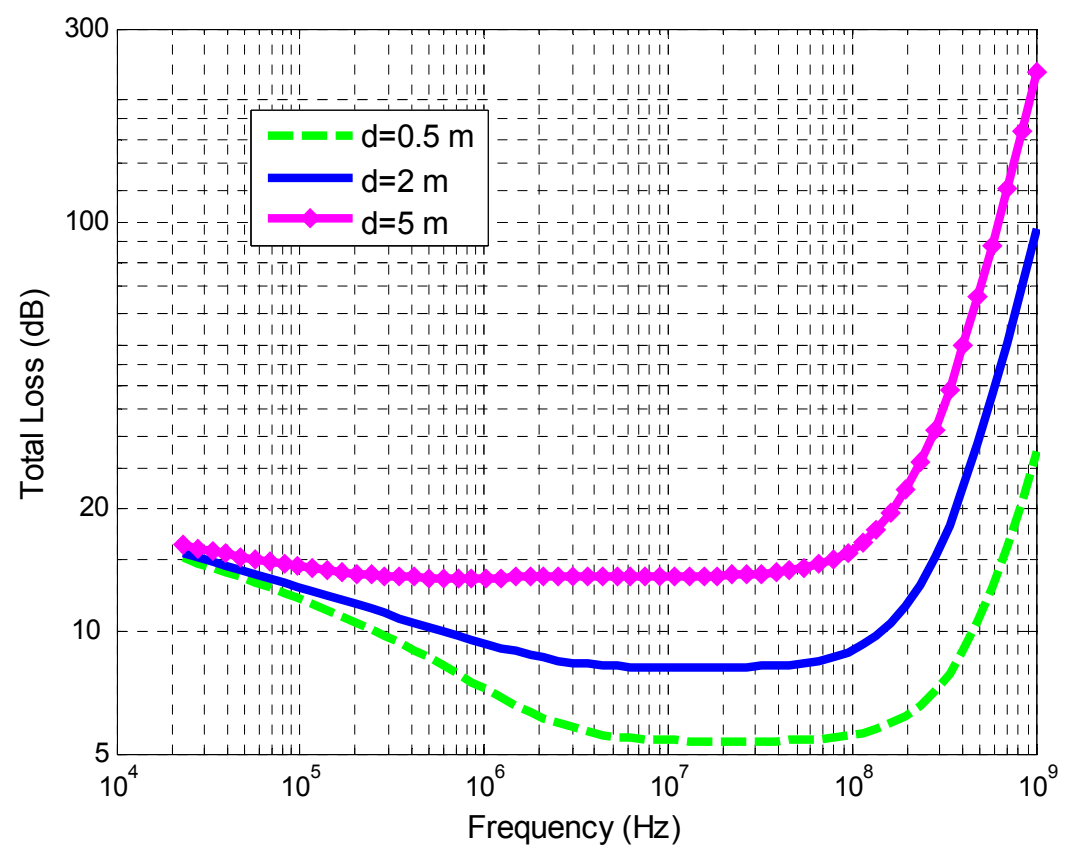

(a) Shallow.

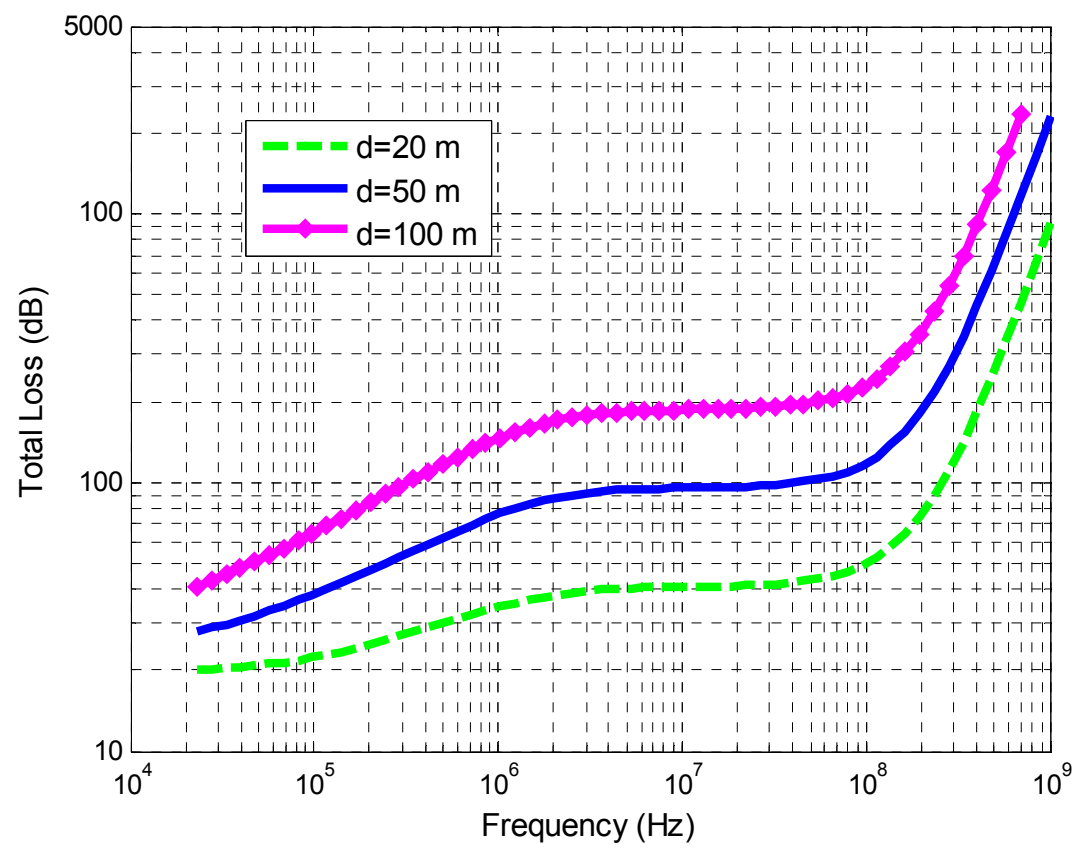

(b) Deep.

Figure 3.14: Total loss for plane wave propagating from air to fresh water. 
Second, we analyze EM waves that propagate from air to soil for wireless underground sensors networks (WUSNs) applications. The realization of WUSNs is challenging since the communications are directly impacted by the characteristics of soil [70]. Variations of soil moisture, temperature and depth can have a significant impact on the attenuation of RF waves traveling to underground sensors. Several analytical and empirical investigations have been performed for improving the performance of aboveground-to-underground (AG2UG) links and underground-to-aboveground (UG2AG) links [71]. Specifically, a maximum communication range of $62 \mathrm{~m}$ was achieved in [72]. However, underground RF communications still suffer from high losses.

The analytical methods presented in section 3.2 can be used to identify optimum conditions for wireless power transmission and communication with underground sensors. The four-component volumetric mixing model [73] describing the frequency-dependent complex permittivity of soil is written as:

$$
\begin{aligned}
\bar{\varepsilon}_{r}^{\alpha}(f)= & \left(\frac{\rho_{d}}{\rho_{s}}\right) \varepsilon_{s}^{* \alpha}+\left(\theta-\delta \rho_{d} A_{e s}\right) \varepsilon_{f w}^{* \alpha} \\
& +\delta \rho_{d} A_{e s} \varepsilon_{b w}^{* \alpha}+\left(1-\frac{\rho_{d}}{\rho_{s}}-\theta\right) \varepsilon_{a}^{* \alpha}
\end{aligned}
$$

where $\varepsilon_{s}^{*}, \varepsilon_{f w}^{*}, \varepsilon_{b w}^{*}$, and $\varepsilon_{a}^{*}$ are the complex dielectric permittivity of soil solids, free water, bound water and air. $\varepsilon_{f w}^{*}$ and $\varepsilon_{b w}^{*}$ are calculated by (3.29). Table 3.3 describes the parameters in the volumetric mixing model presented in [73]. It can be seen from Table 3.3 that six parameters, $\theta, \rho_{d}, A_{e s}, \sigma_{f w}, \sigma_{b w}$ and $\alpha$ determine the dielectric dispersion of soil in the volumetric mixing equation. 
Table 3.3 Parameters in the volumetric mixing model for soil [73].

\begin{tabular}{|c|c|c|}
\hline Volumetric mixing parameters & Range & $\begin{array}{c}\text { Reference } \\
\text { value }\end{array}$ \\
\hline Volumetric water content $\theta_{,} \%$ & $0-100$ & 20 \\
\hline Bulk dry density $\rho_{d,}, \mathrm{~g} \mathrm{~cm}^{-3}$ & $0.5-2.5$ & 1.56 \\
\hline Effective specific surface $A_{e s}, \mathrm{~m}^{2} \mathrm{~g}^{-1}$ & $0-700$ & 100 \\
\hline Dielectric constant of soil particle $\varepsilon_{s}$ & 4.7 & 4.7 \\
\hline Density of soil solid $\rho_{s}, \mathrm{~g} \mathrm{~cm}^{-3}$ & 2.65 & 2.65 \\
\hline Dielectric constant of air $\varepsilon_{a}$ & 1 & 1 \\
\hline Static value $\varepsilon_{d c}$ of free water & 80 & 80 \\
\hline High frequency value $\varepsilon_{\infty}$ of free water & 4.22 & 4.22 \\
\hline $\begin{array}{c}\text { Relaxation frequency } f_{r e l} \text { of free water, } \\
\mathrm{GHz}\end{array}$ & 17.4 & 17.4 \\
\hline Conductivity of free water $\sigma_{f \varepsilon}, \mathrm{S} \mathrm{m}^{-1}$ & $0-2$ & 0.06 \\
\hline Static value $\varepsilon_{d c}$ of bound water & 80 & 80 \\
\hline High frequency value $\varepsilon_{\infty}$ of bound water & 4.22 & 4.22 \\
\hline $\begin{array}{c}\text { Relaxation frequency } f_{r e l} \text { of bound water, } \\
\mathrm{KHz}\end{array}$ & 9 & 9 \\
\hline Conductivity of bound water $\sigma_{b s}, \mathrm{~S} \mathrm{~m}^{-1}$ & $0.1-100$ & 5 \\
\hline Fitting parameter $\alpha$ & $-1-1$ & 0.5 \\
\hline
\end{tabular}

Total losses are plotted in Figure 3.15(a) for three different volumetric water contents of soil at a propagation depth of $0.15 \mathrm{~m}$. Our results illustrate that the total loss increases as the volumetric water content in the soil increases, thereby causing larger power loss. Since the humidity condition of soil can be changed by the weather (i.e., rain or sun exposure, etc.), volumetric water content should be carefully considered for optimum antenna design. Furthermore, Figure 3.15(a) shows the existence of an optimum frequency range. In this optimum range waves experience significantly smaller total loss than in other frequencies. For example, total loss in the frequency range of $10 \mathrm{MHz}$ to $100 \mathrm{MHz}$ for saturated soil with volumetric water content of $40 \%$ is approximately $5-8$ $\mathrm{dB}$ less than the total loss at lowest or highest frequencies in our analysis. This observation is particularly useful for wireless powering underground sensors, where we 
seek to minimize the power transmission loss in order to maximize the power transmission efficiency.

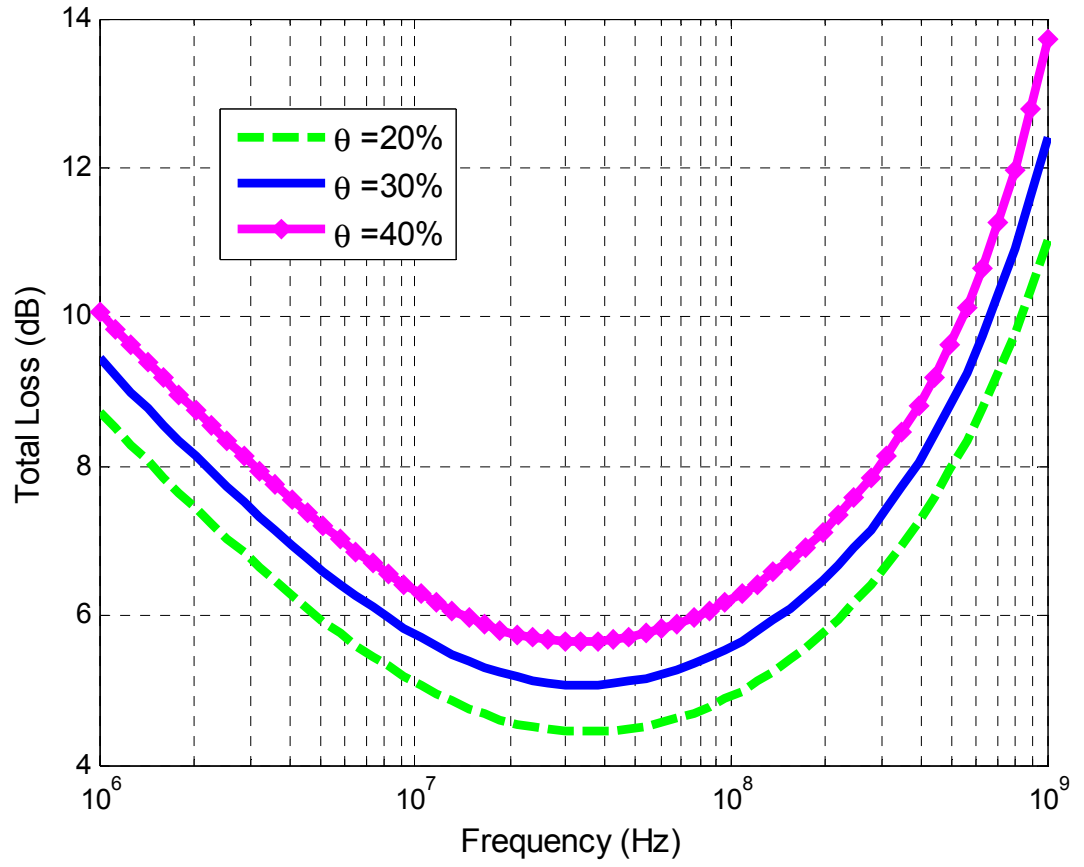

(a) Volumetric water content.

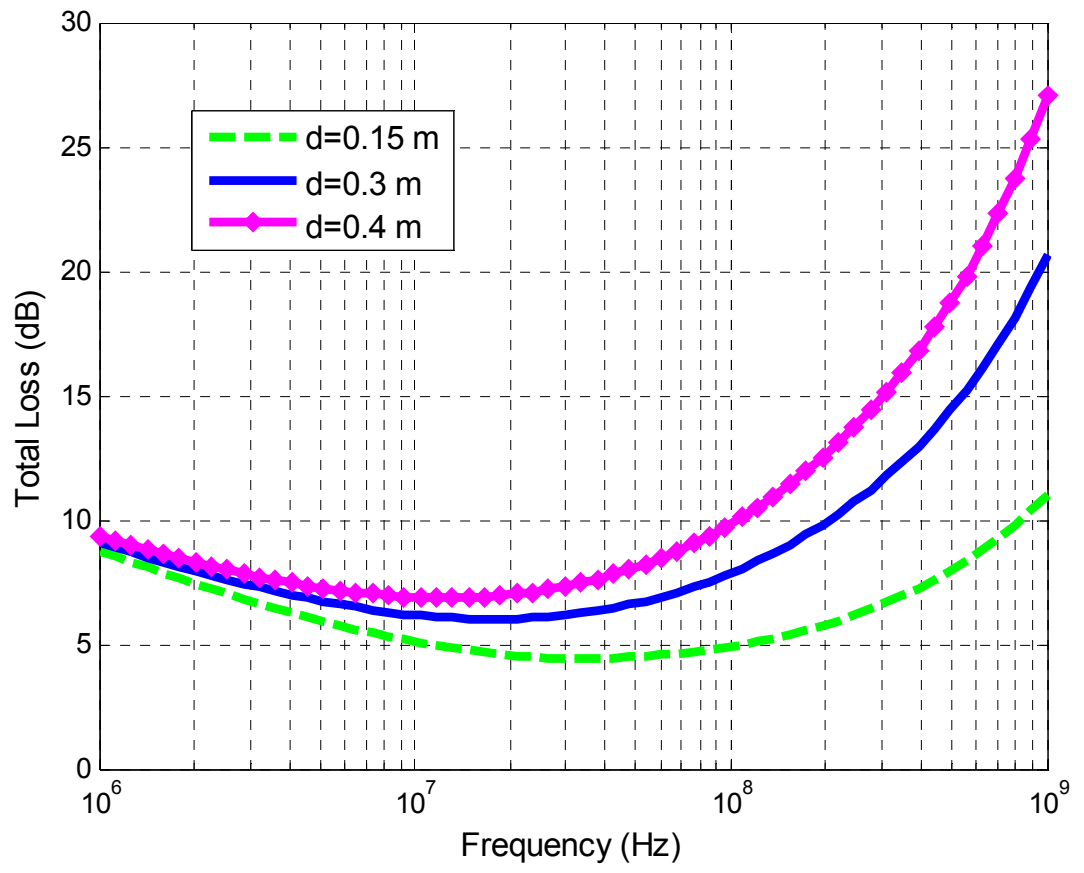

(b) Depth.

Figure 3.15: Total loss for plane wave penetrates through air to soil. 
Sensor installation depths in soil vary for different applications. Therefore, three propagation depths are considered. Figure 3.15(b) illustrates that an optimum frequency range exists when a wave propagates from air to certain depth inside soil. For example, when propagation depth is $0.3 \mathrm{~m}$, the total loss in the $10-30 \mathrm{MHz}$ frequency range is approximately $3 \mathrm{~dB}$ to $15 \mathrm{~dB}$ smaller than the loss at the lowest and highest frequencies of our frequency range. Also, as shown in Figure 3.15(b), the optimum frequency for $0.15 \mathrm{~m}$ depth is around $100 \mathrm{MHz}$, while the one for $0.4 \mathrm{~m}$ is around $10 \mathrm{MHz}$. This shift of the optimum frequency range happens because the propagation loss increases as the depth is increasing while the transmission loss remains the same.

When EM waves propagate from air to soil, power loss is closely related to the electromagnetic properties of soil, operational frequency and wave incident angle. An optimum frequency range of 10-100 MHz was identified for wireless power transmission of underground sensors.

\subsection{Summary}

Plain concrete is widely used for bricks, footing blocks, deck blocks, motorways/roads and airstrips, etc. Developing structural health monitoring systems for plain concrete is of great importance for the protection of lives and infrastructure investments. In this chapter, an optimum frequency range was identified for powering sensors embedded in concrete. Theoretical air-to-concrete plane wave model was used to calculate the transmission loss, propagation loss and total loss for normal incidence and oblique incidence. These losses are closely related to the electromagnetic properties of concrete, operational frequency, and incidence angle. For sensors that are embedded in 
medium depths, maximum power is received when the transmitted wave is normally incident at the air-to-concrete interface and the operational frequency is between 20-80 MHz. This optimum frequency range includes the bands of shortwave radio $(3-30 \mathrm{MHz})$ and VHF TV (54-72 MHz and 76-88 MHz), therefore, embedded sensors operating in the optimum frequency range will be able to efficiently harvest signals in these bands for charging. Also, power transmission through fresh water and soil was investigated as an extension of the theoretical plane wave model. For air-to-water communications, an optimum frequency range between 3-100 $\mathrm{MHz}$ was identified when plane wave propagates to depths less than $5 \mathrm{~m}$. For air-to-soil communications, an optimum frequency range exists between $10-100 \mathrm{MHz}$ when EM waves propagate to depths less than 1 foot in soil. The identified optimum frequency ranges can lead to significant improvement of the wireless powering and/or communication performance of sensors embedded in concrete, fresh water and soil. 


\section{CHAPTER 4}

\section{PLANE WAVE MODEL FOR REINFORCED CONCRETE}

In this chapter, we study the wireless power transmission through reinforced concrete structures. Computational methods are applied to investigate the transmission and reflection coefficients for reinforced concrete slabs as a function of concrete slab thickness and rebar configurations at different frequencies. The electric field induced inside reinforced concrete is also examined. Our results lead to the identification of optimum conditions for wireless power transmission to sensors embedded in reinforced concrete structures.

\subsection{Reinforced Concrete Structure}

In Chapter 3, we presented results involving the wireless power transmission through homogeneous concrete media. In reality and in most applications, reinforced bars are embedded in concrete to improve its mechanical stability, and the applications include bridges/overpasses, architectural structures, concrete walls, footing for gates, poles, dams and parking structures.

A typical reinforced concrete slab is shown in Figure 4.1. It consists of two rebar layers inside the concrete slab, and it is widely used in concrete structures, such as, reinforced concrete walls and bridge decks. Power transmission through this typical reinforced structure is examined in this chapter. Reinforced bars are metallic periodic structures that are expected to significantly affect the propagation of EM waves when

they are inserted into concrete structures. Therefore, rigorous analysis of rebar effects is necessary for the development of optimum wireless powering systems. In this chapter, 
the propagation of EM waves through rebar layers, concrete slabs and reinforced concrete slabs is investigated using computational methods.

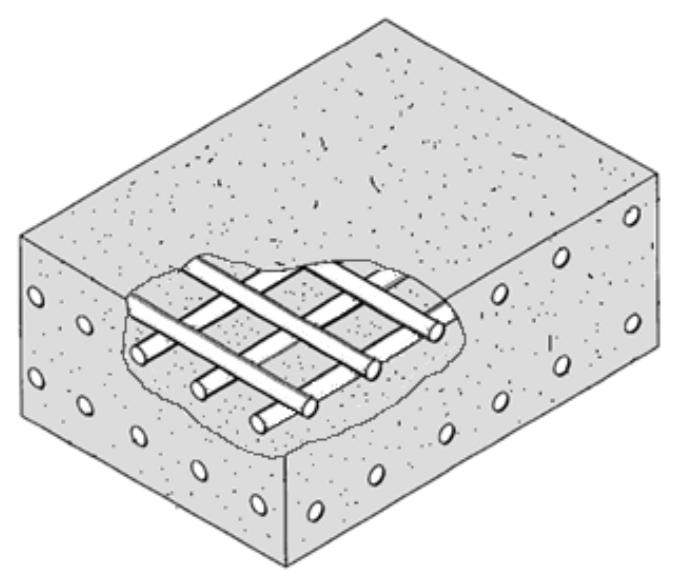

Figure 4.1: Reinforced concrete structure.

\subsection{HFSS Simulation Model}

The propagation of EM waves into reinforced concrete has been studied before using finite-difference time-domain (FDTD) technique, finite-element method (FEM) and the method of moments (MoM). For example, FDTD was used in [74] to examine the reflection and transmission coefficients for reinforced concrete with cross bars in the frequency range of 100-6000 MHz. Various concrete thicknesses and rebar configurations conditions were considered. Scattering from reinforced concrete walls with vertical and crossed bars was analyzed in [75] using FDTD in the frequency range of 0.5-2.0 GHz. The propagation losses in brick and concrete walls were studied at 900 $\mathrm{MHz}$ band using measurements and MoM simulations in [76]. The effects of oblique incidence angels were particularly studied by this work. FEM simulations along with an expansion of EM fields in Floquet modes are used in [77] to study the propagation of EM waves through building walls at $900 \mathrm{MHz}$ and $1.8 \mathrm{GHz}$ for various concrete thicknesses, 
rebar diameters and incidence angles. This work focused on studying the reflection and transmission coefficients for reinforced concrete walls. Specifically, the results of [77] illustrated that plane waves penetrating through reinforced concrete slabs exhibit complicated series of resonances and nulls; the existence of which depends on the permittivity of concrete, concrete slab thickness, rebar period, rebar diameter and incidence angle.

The objective of this chapter is to identify the optimum conditions for wireless powering of sensors embedded in reinforced concrete. Our analysis using EM waves is performed using Ansoft HFSS (High Frequency Structure Simulator). Ansoft HFSS [78] is an industry-standard software for 3D full-wave electromagnetic field simulation and is essential for the design of high frequency and high-speed components based on FEM formulation. Ansoft HFSS is chosen because it includes the necessary functions for our analysis, such as, direct and iterative matrix solvers as well as Floquet ports for periodic structures.

Plane wave penetrating through plain concrete slab, rebar layers and reinforced concrete slab will be examined using Ansoft HFSS simulations in this chapter. Similar geometrical setups are used for all these three cases. Initially, simulation analysis of plain concrete slabs is performed, and the geometry is shown in Figure 4.2. The concrete slab is 8 " thick, 12" wide and it is placed in the center of the model. Vacuum boxes are added above and below the slab. The thicknesses of them are quarter wavelength at the lowest frequency of our simulation range (i.e., $300 \mathrm{MHz}$ ). Notice that a typical thickness of a concrete slab is between 7" and 9". Therefore, a 8" thick slab is analyzed here. Moreover, the width of the slab is 12", which corresponds to the length of the largest 
rebar period (4"-12" as the normal range) that we will analyze. Previous work in [74-77] has used fixed concrete permittivity and conductivity values. Our work implements the extended Debye model into our simulations in order to accurately describe the EM properties of concrete at different humidity conditions.

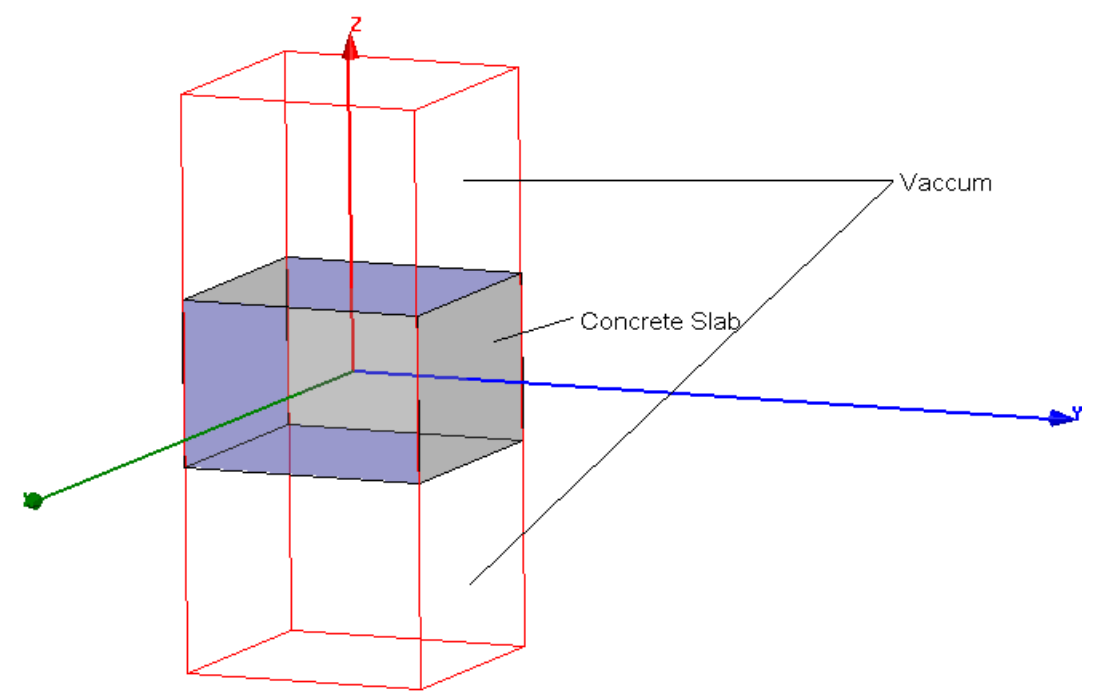

Figure 4.2: Plain concrete slab geometry setup in HFSS.

Both the concrete slab and rebar layers can be considered as periodic structures, so master and slave boundaries are chosen for simulation in HFSS as shown in Figure 4.3. In HFSS, master and slave boundaries are used to model planes of periodicity where the electric field on one surface matches the electric field on another with a phase difference. Specifically, the electric field at each point on the slave boundary is forced to match the corresponding one on the master boundary only with a phase difference. The two boundaries must have the same magnitude, while the direction could be same or opposite. Therefore, it is required that the master and slave boundaries must match with the same geometry, mesh, coordinate system and be assigned to planar surfaces. The setup in 
Figure 4.3 follows these guidelines and it equivalently extends the unit concrete slab to infinity.

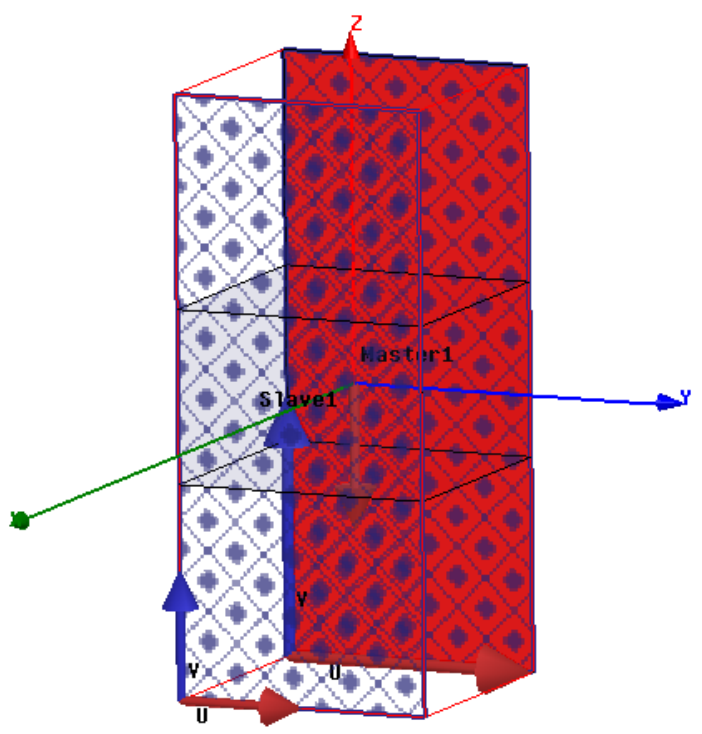

(a)Master and slave 1 .

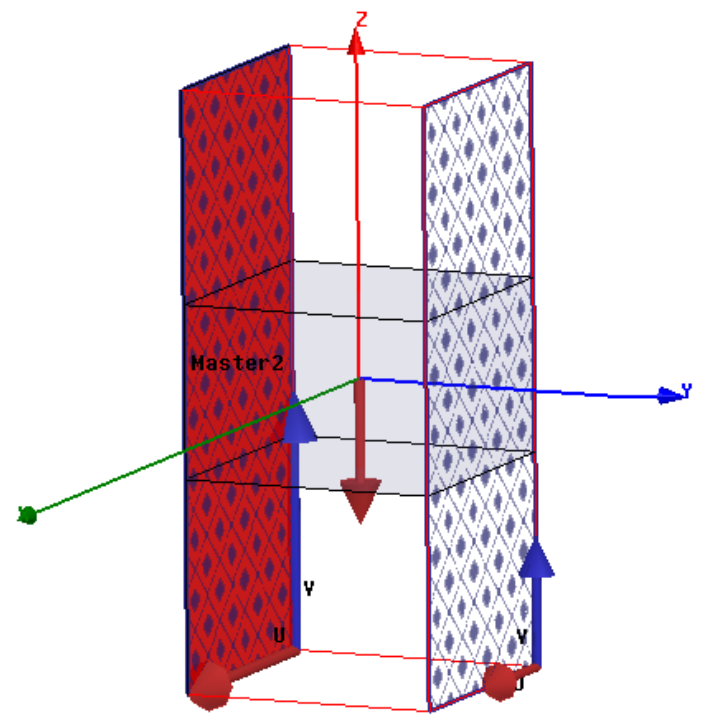

(b) Master and slave 2 .

Figure 4.3: Plain concrete boundary condition setup in HFSS.

Floquet ports are used for the excitation of the plain concrete slab model, as shown in Figure 4.4. A Floquet port in HFSS is exclusively used in planar-periodic structures that are assumed to be infinite in extent. A Floquet port is closely related to a wave port for which a set of modes is used to represent the fields on the port boundary, and these Floquet modes are fundamentally plane waves with propagation direction set by the frequency, phasing, and geometry of the periodic structure. When Floquet ports are used, HFSS solutions include model decompositions that give additional information on the performance of the radiating structure. As shown in Figure 4.4(a), Floquet port 1 is on the top side of the simulation model, with vector $a$ and $b$ along the direction of $\mathrm{x}$ and $\mathrm{y}$ axis. Floquet port 2 corresponds to port 1 and is on the bottom side of the model. Each 
port with two basic modes: $\mathrm{TE}_{00}$ and $\mathrm{TM}_{00}$, and the port fields of the two models are shown in Figure 4.4(b).
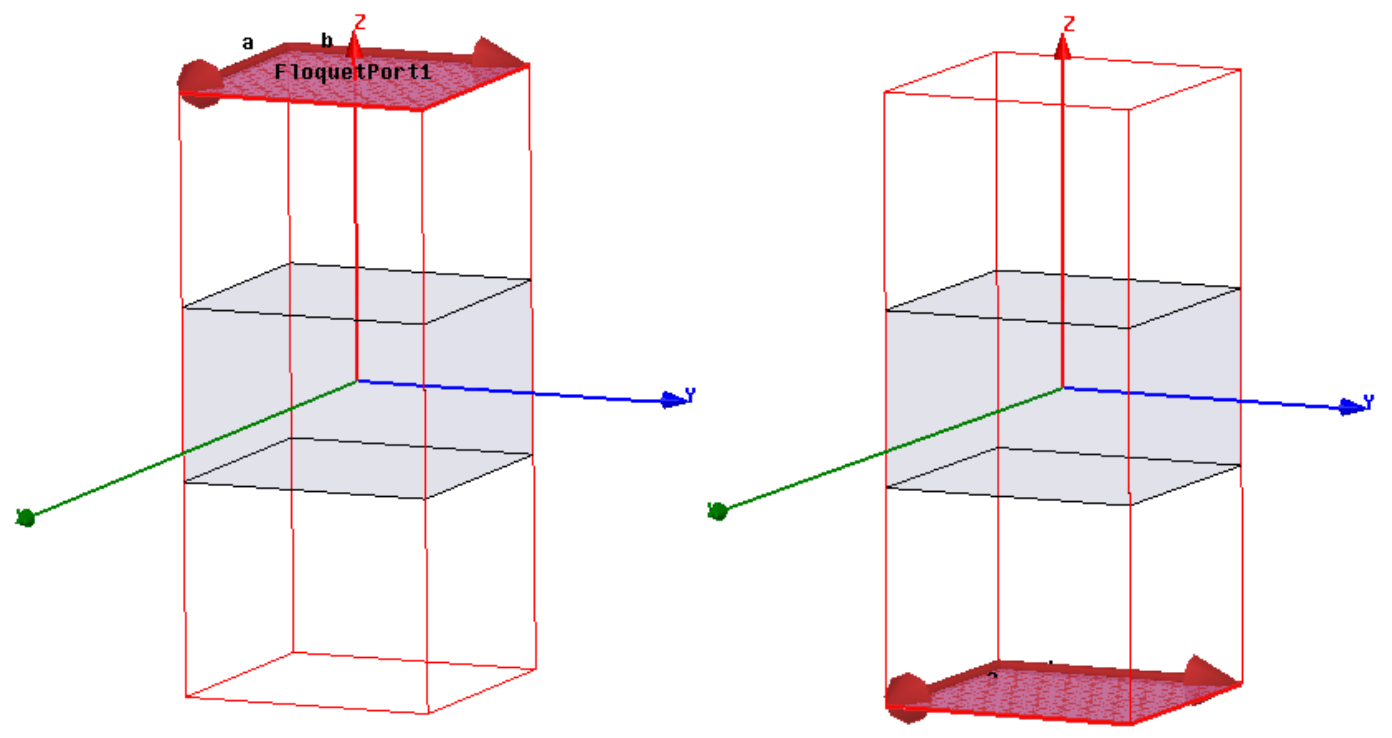

(a) Floquet port.
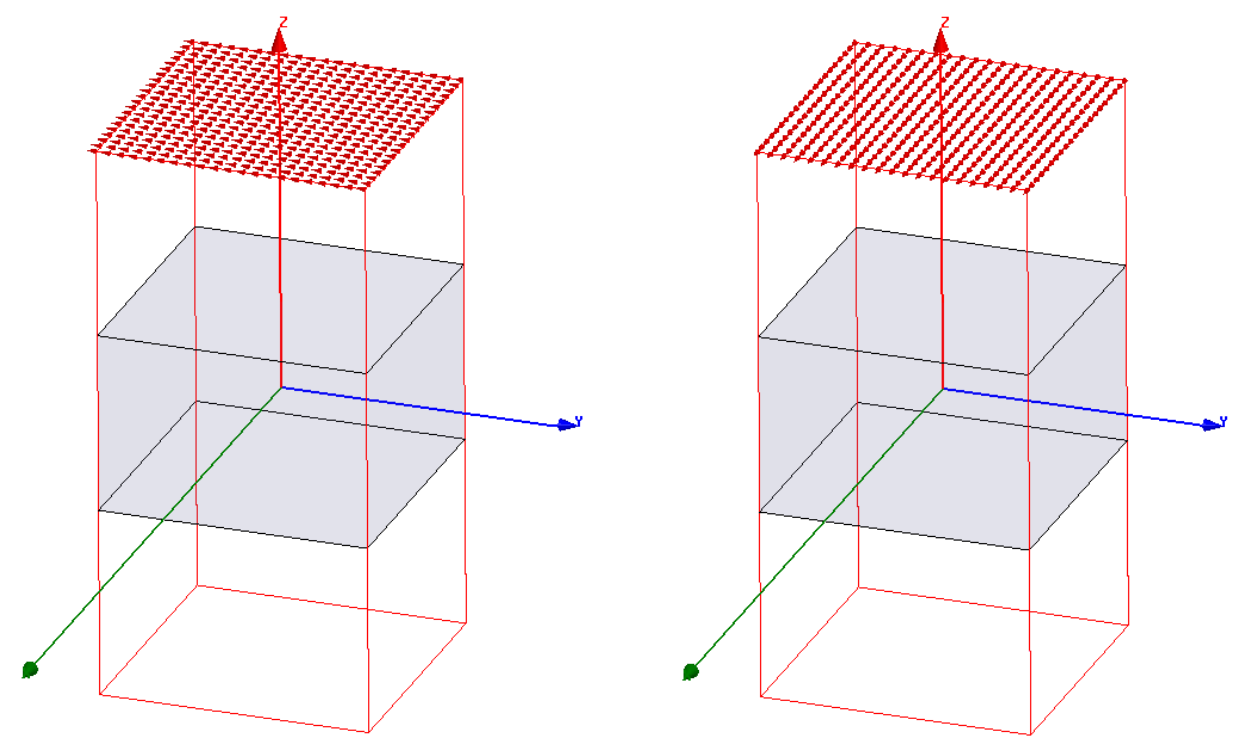

(b) Port modes.

Figure 4.4: Plain concrete excitation setup in HFSS. 
Also, mesh enhancement methods are used to improve the simulation accuracy. One method is to increase the solution frequency, as shown in Figure 4.5(a). For example, when simulating power transmission through concrete slab with $6.2 \%$ humidity, the highest frequency of interest is $1.8 \mathrm{GHz}$. However, when waves penetrate into concrete, the wavelength inside concrete will be smaller than the one in the air, and the FEM mesh must be fine enough in both air and concrete. Therefore, the solution frequency for this simulation setup is increased to $3 \mathrm{GHz}$ in this case to improve the mesh inside concrete, as shown in Figure 4.5(a). Specifically, by increasing the solution frequency we make the mesh finer, thereby improving our simulation accuracy at higher frequencies. The tradeoff of this method is that the FEM matrix size increases, thereby requiring longer simulation time.

Another way to enhance our simulation accuracy is by increasing the number of adaptive passes, as shown in Figure 4.5(b). The convergence criteria in HFSS are based on the S-matrix, and it typically converges prior to field quantities. Therefore, when the absolute field values are of interest, more adaptive passes are needed in order to reach the same convergence as the one obtained for the S-matrix [79]. This means that accurate S parameters do not ensure the accurate values for the electric and magnetic fields. Therefore, the minimum converged passes number is increased to three, as shown in Figure 4.5(b). Also, HFSS solves for the electric fields directly, and then derives the magnetic field from them. Therefore, electric field data are considered to be more accurate than magnetic fields. 


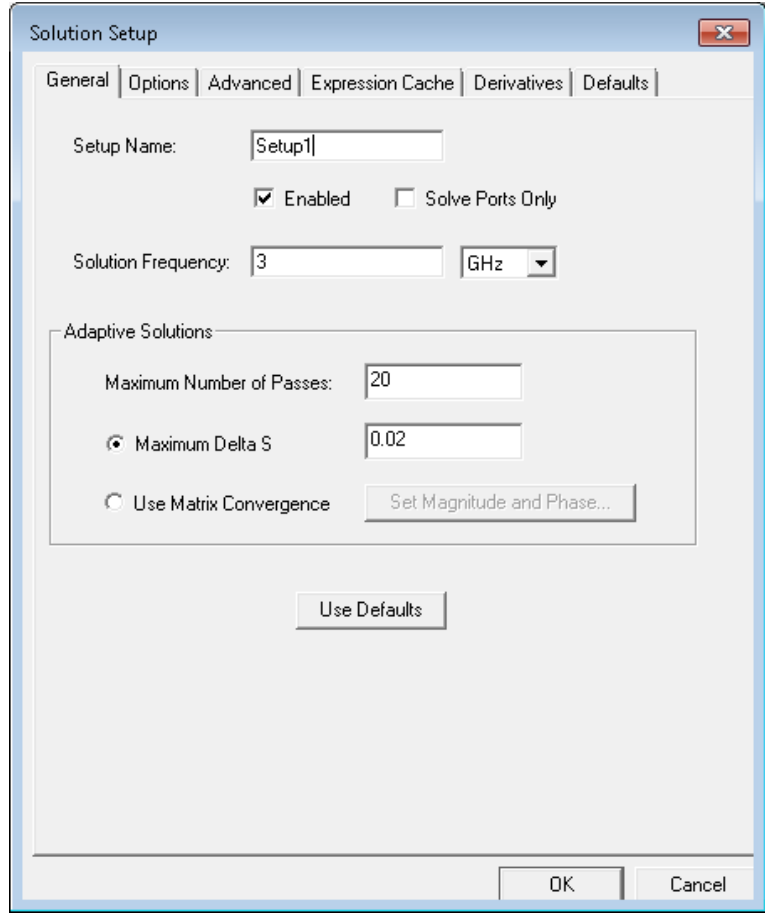

(a) General solution setup.

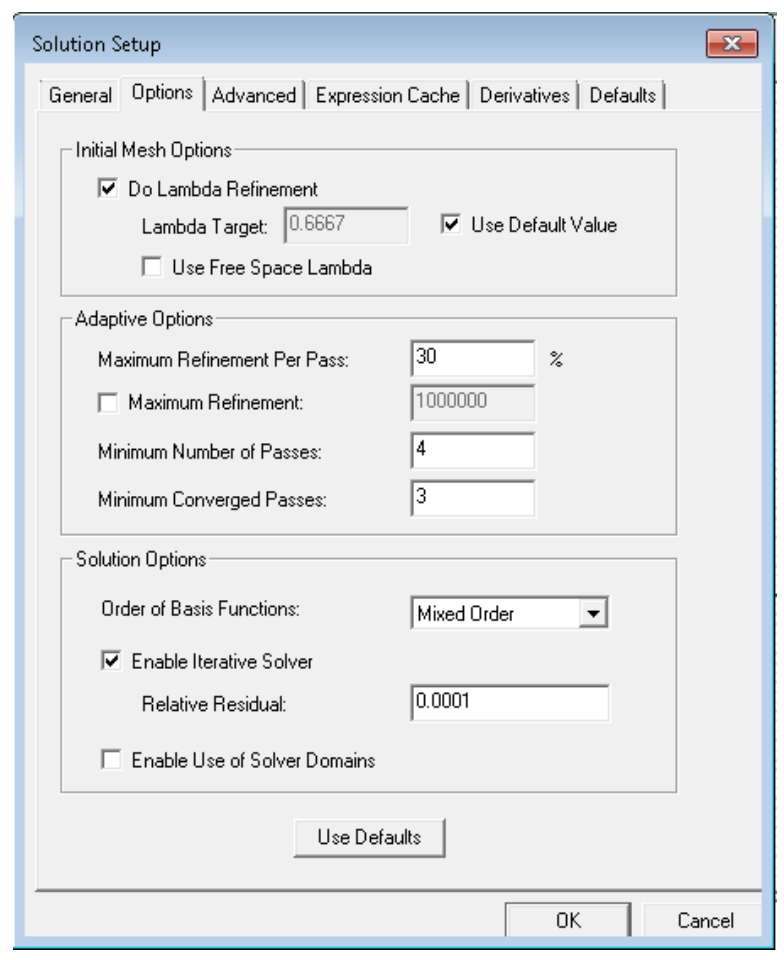

(b) Solution options setup.

Figure 4.5: Plain concrete solution setup in HFSS. 
Besides the methods presented in Figure 4.5, other mesh operations can be used to increase the accuracy of simulations. For example, surface approximations can be used to improve the mesh of curved objects, such as, the round rebars. Also, the model resolution method can also be used to reduce the number of tetrahedrons, using less memory resources while keeping the same accuracy as before.

Using the simulation setup described above, the electric field inside an infinite concrete slab (6.2\% humidity and 8 " thickness) at certain points is initially calculated in HFSS. The results are shown and compared to analytical results in Figure 4.6. This comparison is essential to validate the accuracy of our simulation results. The results shown in Figure 4.6 include the electric field at four different positions: (a) center of the slab, (b) top of the slab in the center, (c) side of the slab $5 \mathrm{~cm}$ away from the center and (d) bottom of the slab in the center. The electric fields are plotted versus the thickness of the concrete slab, $\mathrm{D}=8$ ", normalized by the wavelength inside the concrete $\left(\lambda_{c}\right)$. This is done because the maximum and minimum values of the electric field inside concrete, as well as the reflection and transmission coefficients of the concrete slab are closely related to the electrical thickness of concrete at a specific frequency.

These results illustrate that the accuracy of our simulation is excellent. For example, the largest difference between simulation and theory is only $0.02 \mathrm{~V} / \mathrm{m}$, as shown in Figure 4.6(b). This occurs when the thickness of concrete (i.e., 8") is approximately 2.75 wavelengths, which is electrically very long. Therefore, our simulation model for the concrete slab can be used to accurately calculate the electric field inside concrete slab as well as its transmission and reflection coefficients. 


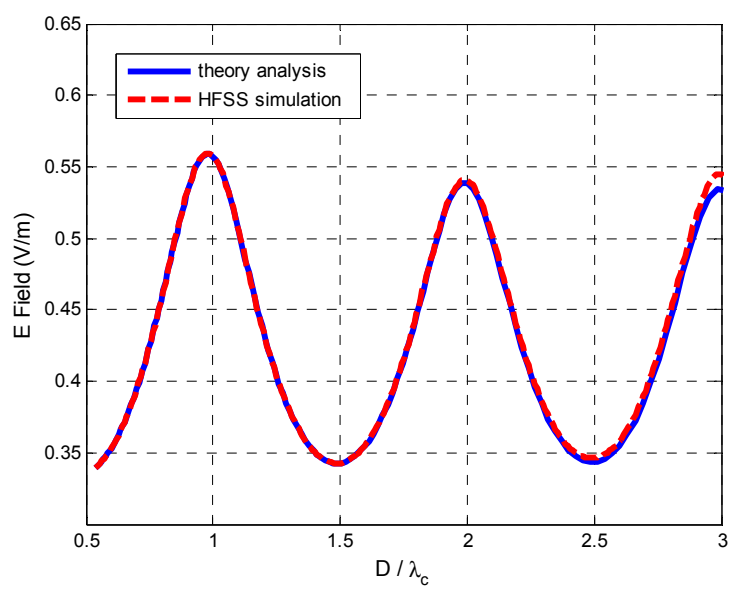

(a) Center $(0,0,0) \mathrm{cm}$

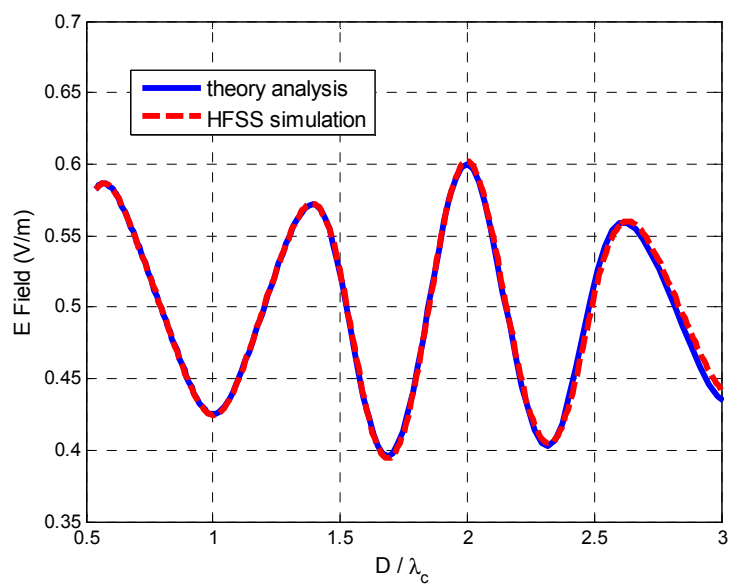

(c) Side $(0,15.24,5) \mathrm{cm}$

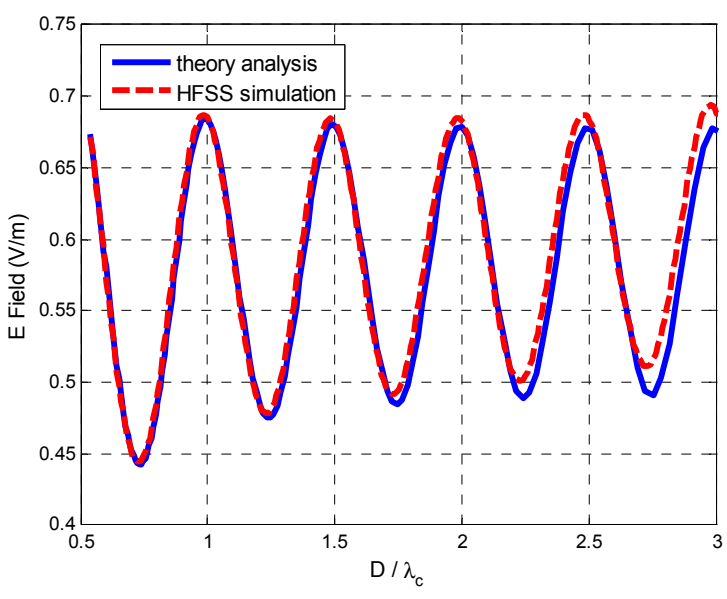

(b) Top $(0,0,10.32) \mathrm{cm}$

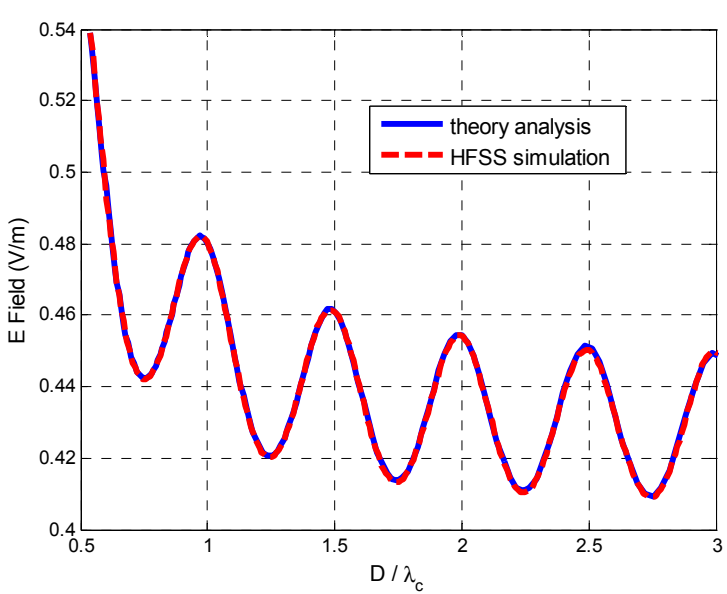

(d) Bottom $(0,0,-10.32) \mathrm{cm}$

Figure 4.6: Comparison of simulated and theoretical results.

Using the same simulation setup in HFSS as shown in Figures 4.2-4.5, reflection and transmission coefficients of an 8 " thick concrete slab at $0.2 \%$ and $6.2 \%$ humidity conditions are calculated. The results are illustrated in Figure 4.7. These parameters are important as they describe the effect of the concrete slab on EM waves and they are closely related to the maximum electric field induced inside the slab. Figure 4.7 illustrates that when the concrete slab thickness is integer multiples of half-wavelength (i.e., $\left.D=n \cdot \frac{\lambda_{c}}{2}, n=1,2,3, \ldots\right)$, maximum transmission occurs through the slab. Also, 
maximum reflection of the slab occurs when the slab's thickness is odd multiples of a quarter wavelength (i.e., $D=n \cdot \frac{\lambda_{c}}{4}, n=1,3,5, \ldots$ ). Also, Figure 4.7 shows that the humidity of concrete affects the amplitude of the reflection and transmission coefficients, but does not affect the occurrence of resonances and nulls at certain wavelengths. For example, as shown in Figure 4.7(b), when the thickness of the concrete is 2.5 times the wavelength inside $0.2 \%$ humidity concrete, the transmission coefficient is about $6 \mathrm{~dB}$ larger than the corresponding value for $6.2 \%$ humidity concrete, due to the larger conductivity of wet concrete that causes larger propagation loss. However, the resonances for both cases still occur at integer multiples of half wavelength ( $\lambda_{c}$ is different in dry and wet concrete).

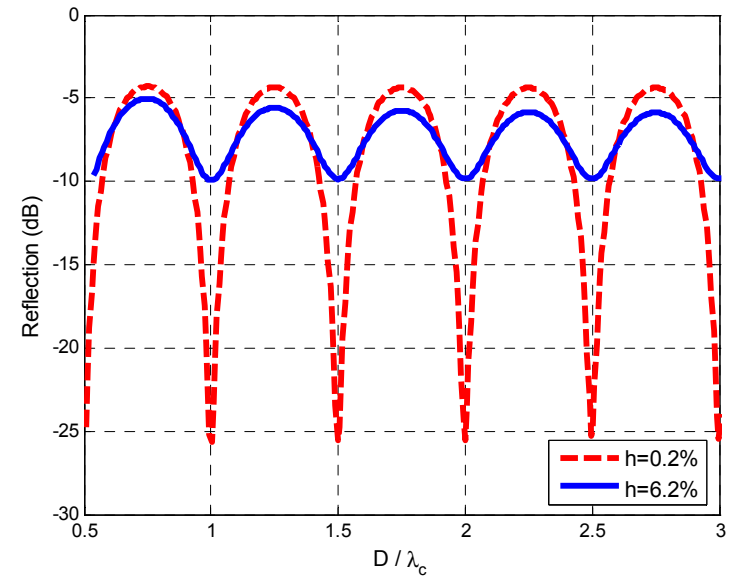

(a) Reflection.

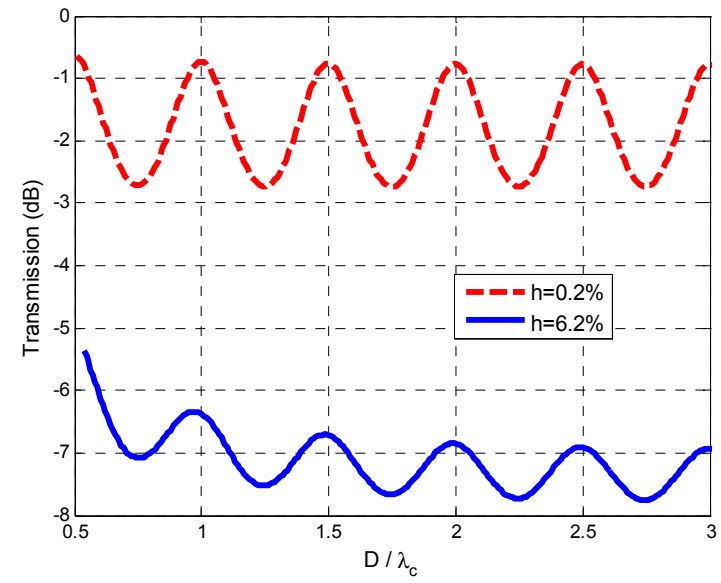

(b) Transmission.

Figure 4.7: Reflection and transmission coefficients for plain concrete slab.

The electric field inside the concrete slab is plotted in Figure 4.8 versus its normalized thickness $\left(D / \lambda_{c}\right)$ and normalized propagation depth $\left(d_{1} / D\right)$ with the wavelength for two humidity conditions. The thickness of concrete slab, $D$, is 8 " and the propagation depth $d_{l}$ increases from 0 " to 8 " to present the variation of the electric field inside the concrete slab. Our results illustrate that the electric field peaks inside the 
concrete slab would occur at certain wavelengths for which the resonances would occur for plane wave penetrating concrete slab. Therefore, when designing wireless power transmission systems for sensors embedded in concrete slabs, the operation frequency and the sensors' depth should be carefully picked to maximize their efficiency.

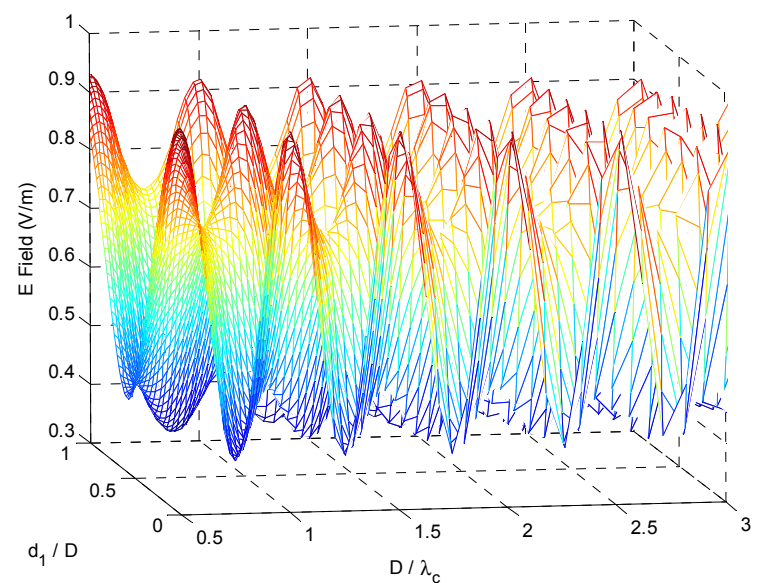

(a) $\mathrm{h}=0.2 \%$.

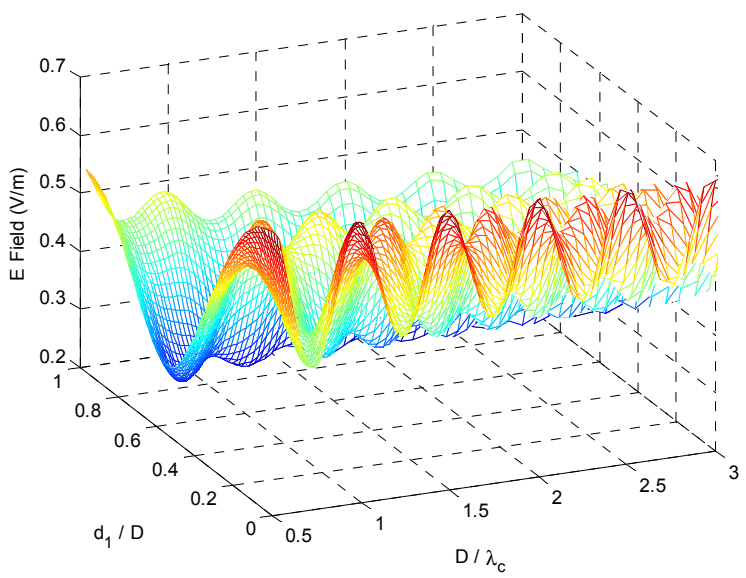

(b) $h=6.2 \%$.

Figure 4.8: Electric field inside an 8" thick concrete slab.

\subsection{Rebar Layers}

In this section, we focus on investigating the effects of rebar layers on EM wave propagation. Reinforced bars are made of steel and they have high strength in tension. Conventional concrete is strong in compression, but weak in tension. Its microscopic rigid lattice will be damaged by appreciable tension (i.e., bending), resulting in cracking and separation. The addition of reinforced bars into concrete helps strengthen the concrete in tension, provides good anchorage to it, thereby increasing the durability of the concrete structure.

Since the invention of reinforced concrete in 1849, various types of reinforced bars are used for construction, such as vertical bars, bonded-junction bars, cross bars, etc. 
Here, we focus on investigating the effects of two layers of cross bars on EM wave propagation. Cross bars are widely used for concrete wall and bridge deck applications as illustrated in Figure 4.1. The geometry of the cross bars is shown in Figure 4.9, where two longitudinal bars (parallel to the travel path of bridge decks or to the upright direction of concrete walls) are bonded with the transverse bars (perpendicular to the travel path of bridge decks or to the upright direction of concrete walls) to form a mesh/lattice layer structure. The geometric parameters of the cross bars' layout include the reinforced bar's diameter, mesh period, and the gap between two layers. These parameters vary in different building structural applications but are generally within the range shown in Figure 4.9.

In our HFSS simulations we choose the following values: mesh period of 6", rebar diameter of (5/8)" and gap between two rebar layers of 3.5" (see Figure 4.10). The material of the reinforced bars is set to be stainless steel in HFSS and their geometry is drawn as cylinders with 12 segments. Also, in order to reduce the mesh number and increase the accuracy, a $2 \mathrm{~mm}$ overlap between the longitudinal and transverse bars of the two layers is implemented in our model.

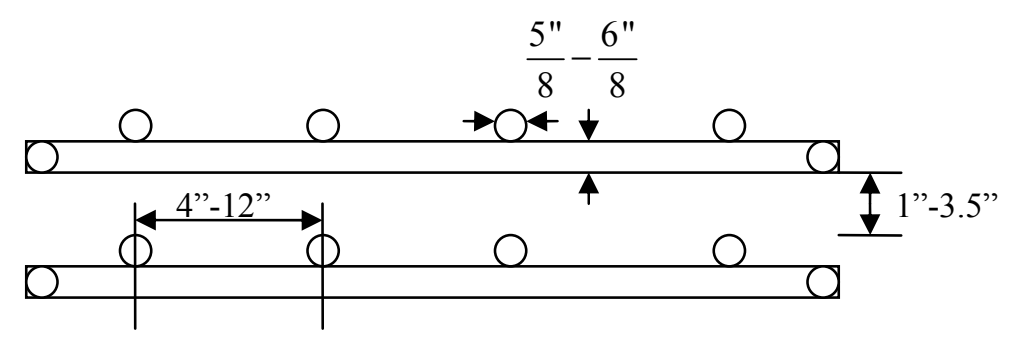

Figure 4.9: Geometry of rebar layers. 


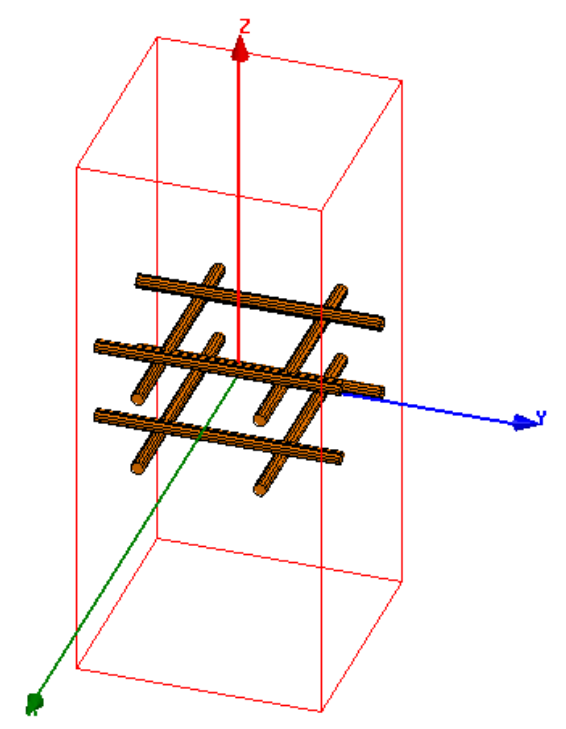

Figure 4.10: Two layers of cross rebars in HFSS.

The reflection and transmission coefficients for an EM wave impinging on the two rebar layers are calculated using HFSS. The results are plotted versus the mesh period, $g$, normalized with the wavelength in Figure 4.11. The effects of rebars on EM waves are first examined without the presence of concrete in order to separate them from the effects of the concrete slab. The results in Figure 4.11 illustrate that the resonances occur when $g \approx n \cdot \frac{\lambda_{0}}{2}, n=1,2,3, \ldots$, where $\lambda_{0}$ is the wavelength in free space and $g$ is the mesh period. It should be pointed out that the first resonance occurs when the mesh period is close to half wavelength. Also, Figure 4.11 shows that when mesh period $g$ is less than a half wavelength, EM waves are dramatically shielded by two layers cross rebar structure. Typically, the mesh period, $g$, takes values in the range of 4"-12". Therefore, only waves with wavelengths smaller than approximately 8 " are able to efficiently propagate through cross rebars. In fact, rebar layers perform as frequency 
selective surfaces (FSS) that allow the wave penetration of certain frequencies and incident angles and block others.

Moreover, Figure 4.11 shows that the transmission and reflection properties of the rebar layers become quite complex when mesh period is longer than one wavelength (i.e., $\mathrm{g}>\lambda_{0}$ ). For example, when the mesh period, $g$, is between 2 and 2.5 wavelengths, more than ten resonances and nulls occur in the transmission and reflection coefficient. It can be concluded that wave propagation is dramatically affected by rebar mesh layers.

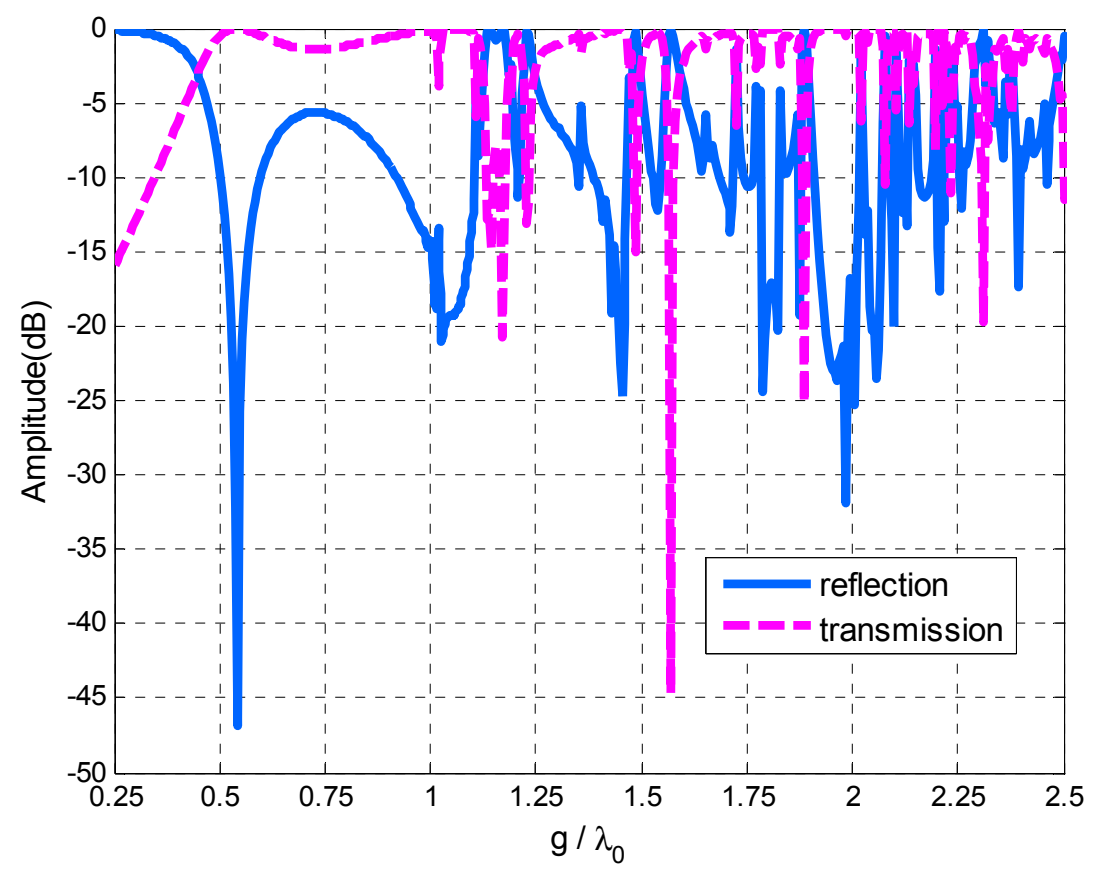

Figure 4.11: Reflection and transmission coefficients for two layers cross rebars.

The electric field distribution inside and around the two rebar layers is also plotted in Figure 4.12. Specifically, two frequencies are considered: $1.07 \mathrm{GHz}$ and 600 $\mathrm{MHz}$, which correspond to $\mathrm{g} / \lambda_{0}=0.54$ and $\mathrm{g} / \lambda_{0}=0.30$. The results of Figure 4.12 illustrate that the frequency of $1.07 \mathrm{GHz}$ (i.e., $\mathrm{g} / \lambda_{0}=0.54$ ) corresponds to the first resonance in Figure 4.11, where the transmission coefficient exhibit a peak. Therefore, at this 
frequency the wave penetrates efficiently through the rebar layers. On the contrary the second frequency of $600 \mathrm{MHz}$ (i.e., $\mathrm{g} / \lambda_{0}=0.30$ ) corresponds to a null of the reflection coefficient. Therefore, at this frequency the wave is blocked by the rebar layers. Figure 4.12 confirms our expectations, as it clearly illustrates that at $1.07 \mathrm{GHz}$ the waves efficiently penetrate through the rebars whereas at $600 \mathrm{MHz}$ the waves are blocked by them. The results in Figure 4.12 also illustrate that the longitudinal bars of the two layers perform the significant shielding of the incident wave, while the transverse bars below them have less effects on the wave propagation. For example, the smallest electric field in Figure 4.12(b) is around the longitudinal bars. This is expected, since as the wavelength increases, the longitudinal bars of the upper layer shield the waves and allow less penetrating. Therefore, in order to optimize wireless powering systems, the polarization of antennas should be parallel to the direction of the longitudinal bars.

Furthermore, at the resonant frequency of $1.07 \mathrm{GHz}$, the strongest field is induced between the two rebar layers [see Figure 4.12(a)]. Therefore, the positions between the rebar layers are more suitable for the placement of SHM sensors, since more power can be received at these positions. Also, sensors buried between the two rebar layers can be protected from damage when concrete is poured. For example, if sensors are installed on top of the rebar layers, they can be potentially damaged by workers stepping on them when they are pouring the concrete.

In conclusion, the propagation of EM wave through rebar layers is affected by the mesh period, diameter of the bars and the gaps between the rebar layers. The mesh period is the dominant parameter. In fact, when the mesh period, $g$, is less than a half 
wavelength, the rebars form a very effective EM shield that can dramatically reduce the power transmission efficiency.
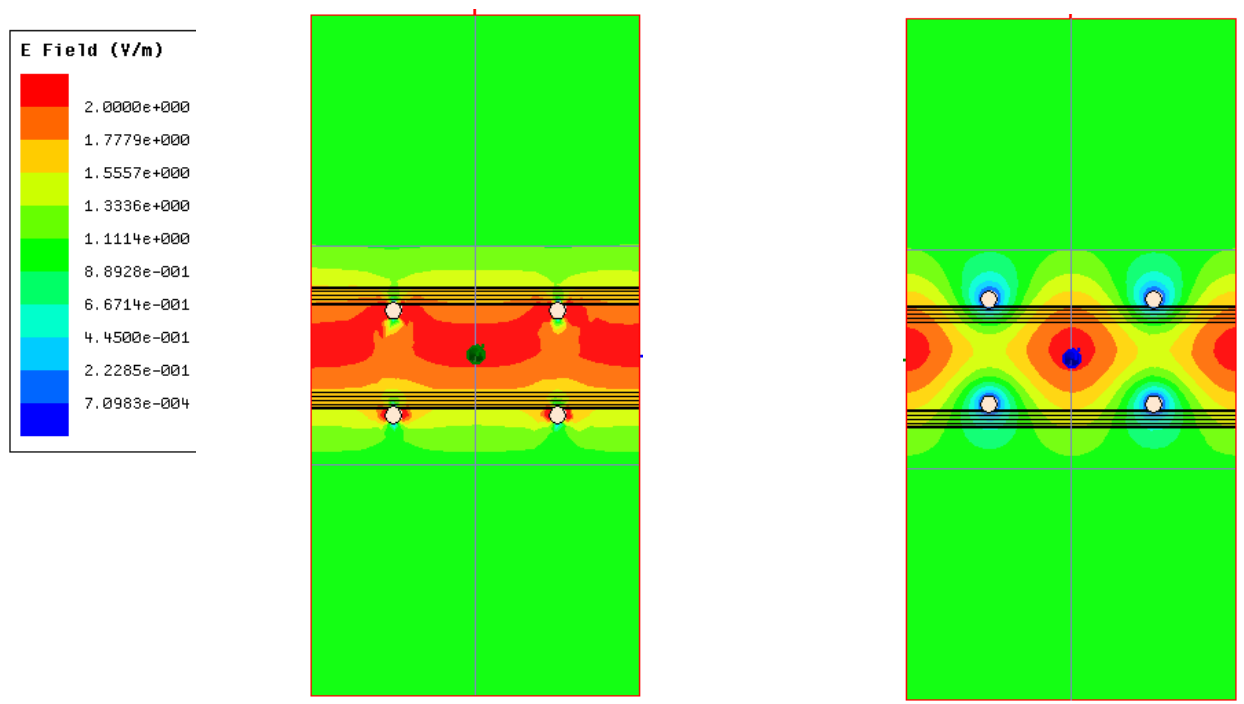

(a) yz-plane view for $1.07 \mathrm{GHz}\left(\mathrm{g} / \lambda_{0}=0.54\right)$. (b) xz-plane view for $1.07 \mathrm{GHz}\left(\mathrm{g} / \lambda_{0}=0.54\right)$.
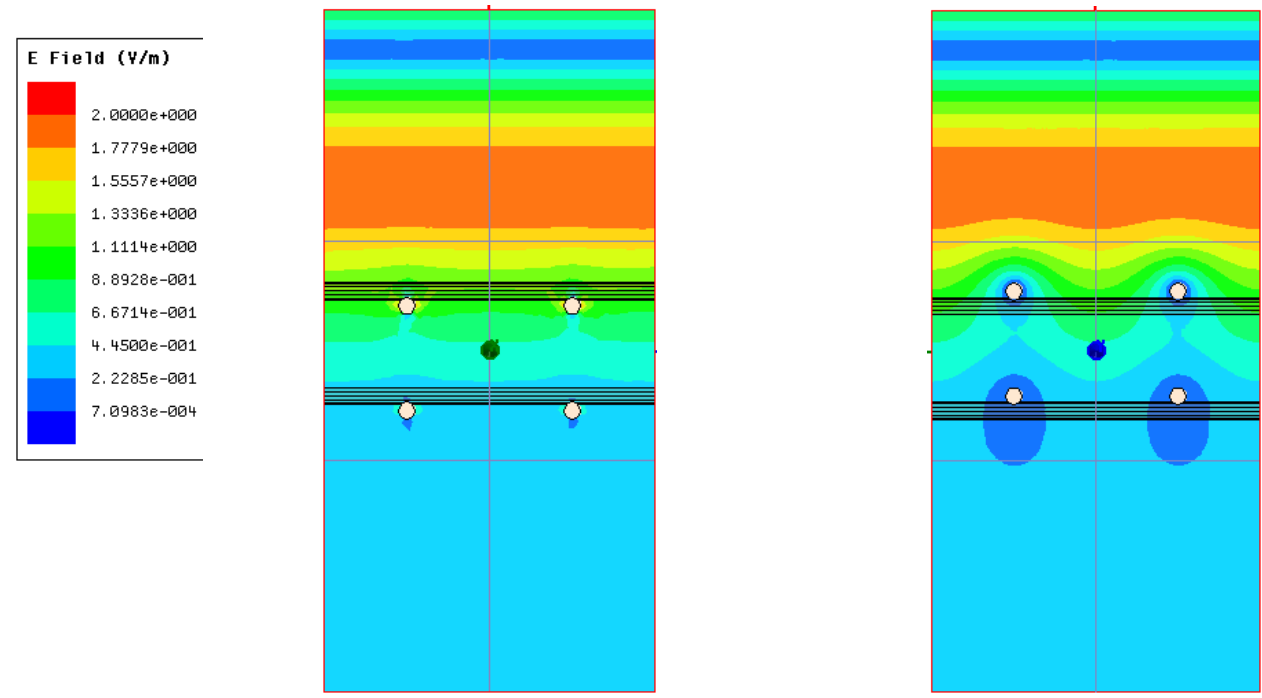

(c) yz-plane view for $0.6 \mathrm{GHz}\left(\mathrm{g} / \lambda_{0}=0.30\right)$.

(d) Xz-plane view for $0.6 \mathrm{GHz}\left(\mathrm{g} / \lambda_{0}=0.30\right)$.

Figure 4.12: Electric field distribution for two layers cross rebars. 


\subsection{Reinforced Concrete Slab}

Based on the discussions of EM waves penetrating through plain concrete slab and two layers of cross rebars, the reinforced concrete slab model is analyzed in this section. The geometry of a typical reinforced concrete slab is shown in Figure 4.13, corresponding to the bridge deck/reinforced concrete wall shown in Figure 4.1. In Figure 4.13, $r$ refers to the diameter of the reinforced bars, $g$ presents the mesh period, $D$ is the thickness of the concrete slab and $l$ refers to the depth of the upper and lower mesh layers' depths inside the slab. The geometry parameters of the reinforced concrete slab vary for different applications. Typical values of the parameter are as follows: $D$ ranges between 7" and 9", $r$ ranges between (5/8)" and (6/8)", $g$ ranges between 4 " and 12", $l$ ranges between $1.5 "$ and $2 "$.

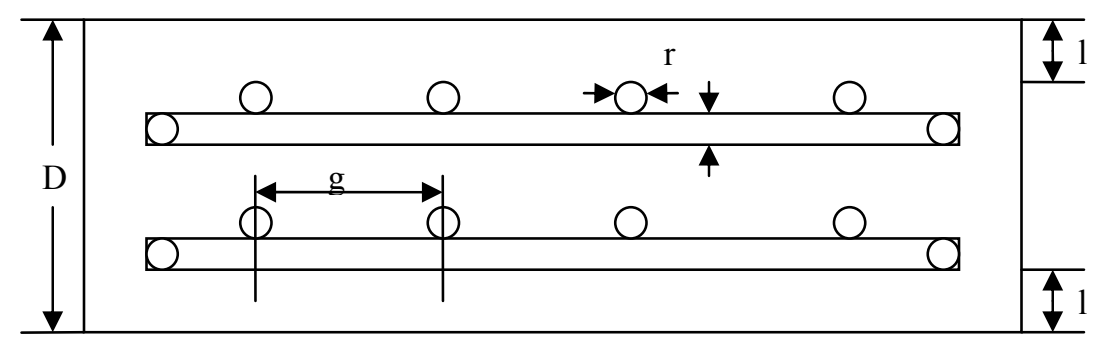

Figure 4.13: Geometry of reinforced concrete slab.

\subsubsection{Simulation Model}

The simulation setup of the reinforced concrete slab is shown in Figure 4.14. This geometry is the combination of the concrete slab and the two layers cross rebars' simulation models, as shown in Figure 4.2 and 4.10. In Figure 4.14(a), the concrete slab thickness $D$ is 8 ", the mesh period $g$ is 6", the diameter of the rebar $r$ is $(5 / 8)$ ", and the mesh layers' depths $l$ is 1.5 ". The width of the concrete slab is 12 ", two times the period 
of the lattice. Two Floquet ports are set on top and bottom sides of the simulation model as excitations. Master/slave boundaries are applied to extend this periodic structure to infinity. In Figure 4.14(b), all parameters are kept the same except the mesh period, $g$, which is increased to 12 " in order to examine the effects of various mesh periods on power transmission.

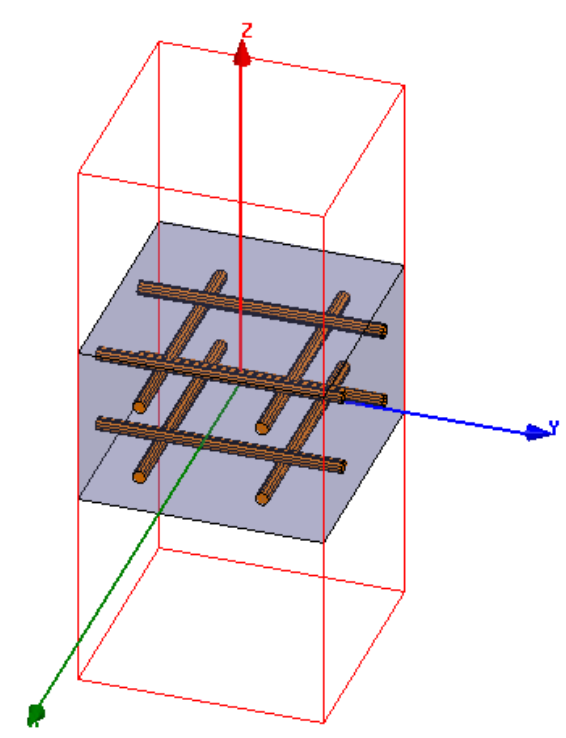

(a) Rebar period is 6".

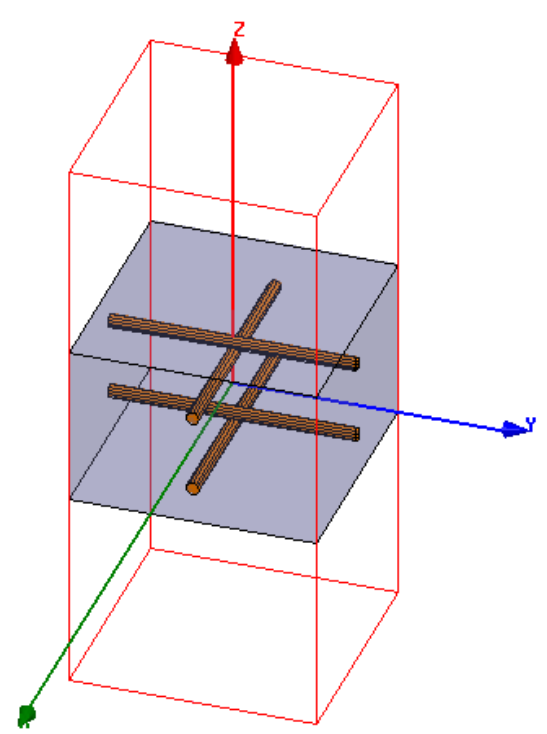

(b) Rebar period is $12 "$.

Figure 4.14: Reinforced concrete simulation setup in HFSS.

\subsubsection{Effects of Concrete Slab}

The reflection and transmission coefficients of EM waves impinging on the reinforced concrete slab of Figure 4.14 are calculated using Ansoft HFSS. The results are shown in Figure 4.15. In order to compare these results with the ones obtained before for the two rebar layers in free space, the reflection and transmission coefficients for reinforced bars with mesh period of 6" in free space are also plotted in Figure 4.15. In the plots of Figure 4.15, the concrete thickness $D\left(8^{\prime \prime}\right)$ is normalized with the wavelength. 
The results of Figure 4.15 illustrate that when EM waves penetrating a reinforced concrete slab they are affected by the combination of concrete slab and rebar layers. Specifically, for the plain concrete slab case, resonance (maximum transmission) occurs when $D$ is integer multiple of half-wavelength $\left(D=n \cdot \frac{\lambda_{c}}{2}, n=1,2,3, \ldots\right)$, as shown in Figure 4.7. However, in Figure 4.15(a), the resonance at $D=\frac{\lambda_{c}}{2}$ does not occur for either dry or wet concrete because the rebar layers inside the concrete slab shield the wave at that wavelength, as shown in Figure 4.15(c). Also, even though a resonance does not occur when $\mathrm{D}=\lambda_{\mathrm{c}}$ in the case of rebar layers in free space, a resonance occurs for the reinforced concrete slab [see Figure 4.15(a)]. This resonance is caused by the effects of plain concrete slab [see Figure 4.7(a)]. Therefore, it can be concluded that when analyzing power transmission through reinforced concrete, the effects of both concrete slab and reinforced bars must be carefully considered.

Furthermore, even though both the concrete slab and the rebar layers affect the power transmission, Figure 4.15 illustrates that for $0.2 \%$ humidity reinforced concrete, the effects of the mesh layers dominate the EM wave propagation. In addition, for $6.2 \%$ humidity reinforced concrete, the concrete slab dominates the EM wave propagation. For example, the reflection coefficient for $6.2 \%$ humidity reinforced concrete [see Figure 4.15(a)] closely resembles the one for $6.2 \%$ humidity concrete slab [see Figure $4.7(\mathrm{a})$ ] with resonances occurring at $D=n \cdot \frac{\lambda_{c}}{2}, n=1,2,3, \ldots$ However, the reflection coefficient for $0.2 \%$ humidity reinforced concrete [see Figure $4.15(\mathrm{a})$ ] closely resembles the one for 
the two layers of cross rebars in free space [see Figure 4.15(c)], exhibiting complex resonances and nulls as $\mathrm{D} / \lambda_{\mathrm{c}}$ increases.

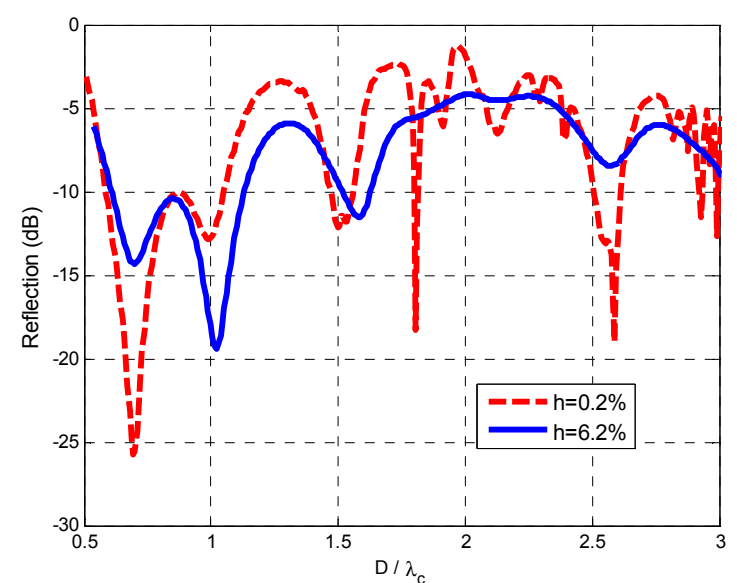

(a) Reflection for reinforced concrete slab.

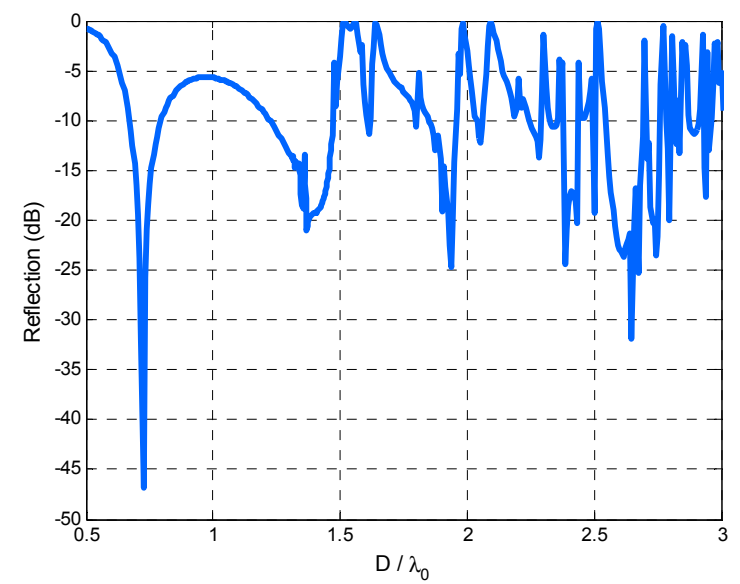

(c) Reflection for rebar layers in free space.(d) Transmission for rebar layers in free space.

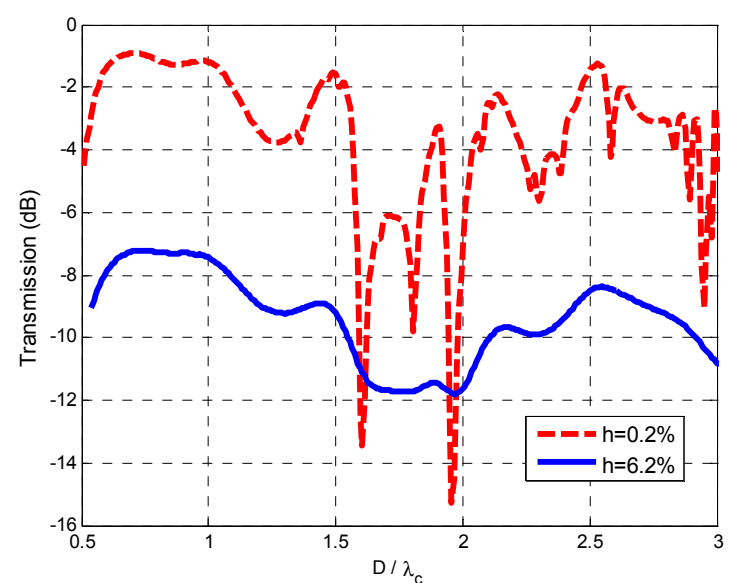

(b) Transmission for reinforced concrete slab.

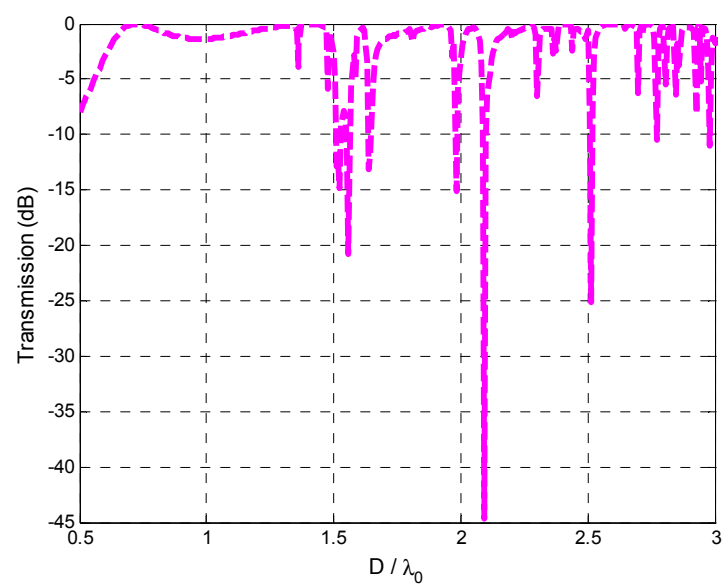

Figure 4.15: Reflection and transmission coefficients.

Figure 4.15(a) and (b) also illustrate that the power transmission is significantly affected by the conductivity of concrete. For instance, the transmission coefficient for reinforced concrete slab with $6.2 \%$ humidity is approximately $6 \mathrm{~dB}$ less than the one for $0.2 \%$ humidity reinforced concrete slab [see Figure 4.15(b)]. Therefore, our study will focus on analyzing EM wave propagation from air into wet reinforced concrete slab with 
$6.2 \%$ humidity. This is done because in most cases, EM waves will propagate more efficiently through dry concrete than through wet concrete. However, optimum power transmission conditions for dry concrete may not ensure optimum performance for wet concrete conditions. Also, as described in Chapter 3, the SHM sensors are buried inside concrete during the construction period, and may need to perform monitoring even when the concrete is still wet. Therefore, the study of EM wave propagation in wet reinforced concrete is necessary.

The maximum electric field induced inside a $6.2 \%$ humidity reinforced concrete versus the thickness of the slab, $D$, normalized with wavelength is shown in Figure 4.16. These results illustrate that when concrete slab thickness $D$ is approximately 1.4 to 1.7 wavelengths in wet concrete, the electric field inside the slab peaks. In this range, resonances occur for both rebar layers and concrete slab [see Figure 4.15 and 4.7], therefore peak electric field is induced inside the reinforced concrete slab. It should be pointed out that the resonances and nulls in Figure 4.7 and Figure 4.15(c) and (d) are not perfectly match the ones in Figure 4.15(a) and (b), since the rebar layers are with depth $l$ of 1.5" inside concrete slab, as shown in Figure 4.13. Waves suffer from transmission and propagation losses at the air-to-concrete interface and depth $l$ before they reach the rebar layers inside concrete slab, thereby causing the mismatch.

From the analysis above, we can expect an optimum frequency range for power transmission through reinforced concrete slab with following information considered: (a) resonances occur for plain concrete slab when concrete thickness $D=n \cdot \frac{\lambda_{c}}{2}, n=1,2,3, \ldots$,

(b) resonances occur for rebar layers when mesh period $g \approx n \cdot \frac{\lambda_{c}}{2}, n=1,2$, (c) peak 
electric field induced inside reinforced concrete slab when $g /\left(\lambda_{c} / 2\right) \approx D /\left(\lambda_{c} / 2\right)=1,2,3$, (d) concrete slab thickness $D$ ranges between 7" and 9", mesh period $g$ ranges between 4" and 12", and (e) EM waves are dramatically shielded by two layers cross rebar structure when mesh period $g$ is less than a half wavelength. It can be calculated from the conditions above that electric field peaks inside reinforced concrete slab would occur when the wavelength inside concrete $\lambda_{c}$ varies in the $3.5 "-8$ " range, approximately corresponds to $600 \mathrm{MHz}-1.3 \mathrm{GHz}$ frequency range in free space. It should be pointed out that this frequency range includes the $902-928 \mathrm{MHz}$ ISM band, so antennas working in this ISM band can be used for power transmission. Antennas operating within this frequency range can achieve larger power transmission efficiency than other frequencies we have examined. Also, antenna's performances are not sensitive to changes of the geometrical configurations of the reinforced concrete slab when operating in this frequency range.

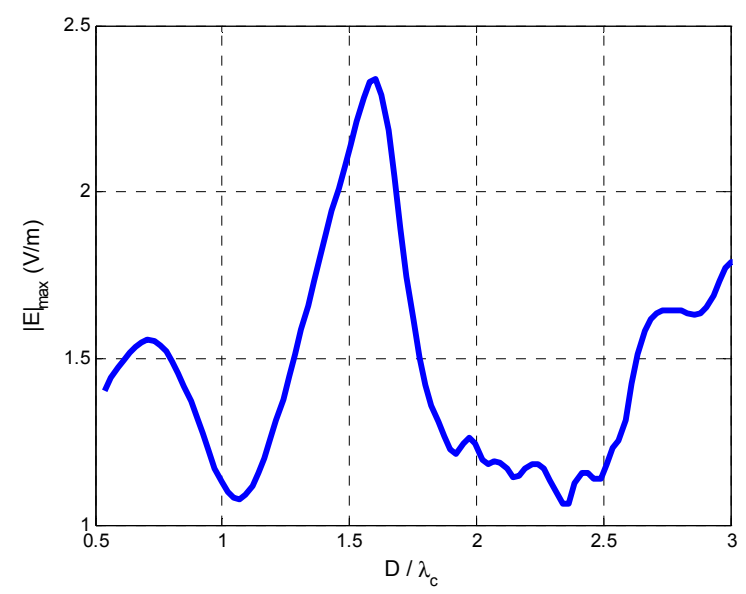

Figure 4.16: Maximum electric field induced inside reinforced concrete slab. 


\subsubsection{Effects of the Configuration of Rebars}

In practice, the diameter, mesh period, and positions of rebars inside a concrete slab can vary significantly. In this section the effects of rebar layers' configurations on the performance of wave propagation are examined. The simulation setup of this case is shown in Figure 4.14 as reference. In this reference case, the mesh period $g$ is 6", rebar diameter $r$ equals to (5/8)", mesh depth inside concrete slab $l$ equals to 1.5 ", moisture content of the concrete is $6.2 \%$. Here, the configuration of the rebars is changed by varying one geometrical parameter at a time. Our simulation results will be compared with the results of the reference case. Our study aims to identify which configuration parameters dominate the effects of wave propagation through reinforced concrete. Investigating the effects of these parameters will lead to optimum design of wireless powering systems for SHM embedded sensors.

The reflection and transmission coefficients are calculated for varying geometrical parameters and the results are shown in Figure 4.17. First, the reflection and transmission coefficients of reinforced concrete slab for two mesh period $g$ are shown in Figure 4.17(a). Our results illustrate that when mesh period is varied to 12 ", the reflection coefficient of the reinforced concrete slab closely resembles the one for $6.2 \%$ humidity concrete slab [see Figure 4.7(a)] with resonances occurring at $D=n \cdot \frac{\lambda_{c}}{2}, n=1,2,3, \ldots$ This is expected, since the first two resonances of rebar layers with 12 " mesh period would occur when $D \approx n \cdot \frac{\lambda_{c}}{2}, n=\frac{2}{3}$ and $\frac{4}{3}$ (expected from $\left.g \approx n \cdot \frac{\lambda_{c}}{2}, n=1,2\right)$, and there will not be co-resonance for rebar layers and concrete slab. Therefore, effects of concrete slab dominate the EM wave propagation. 

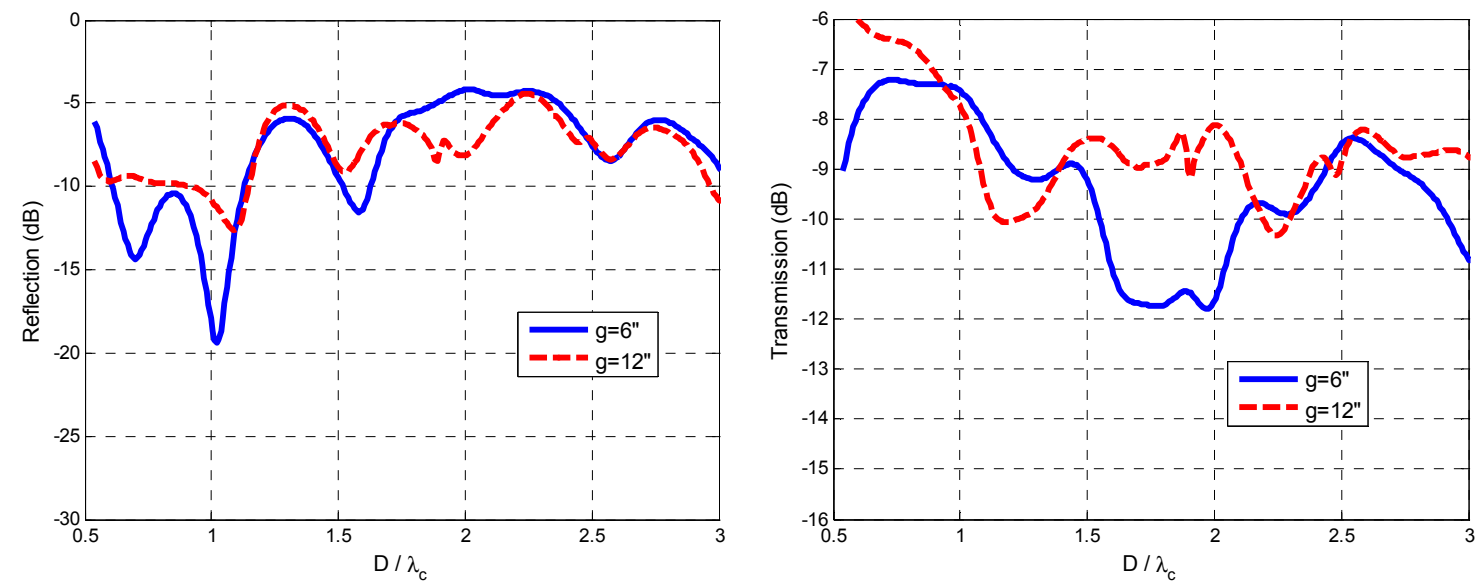

(a) Rebar period g.
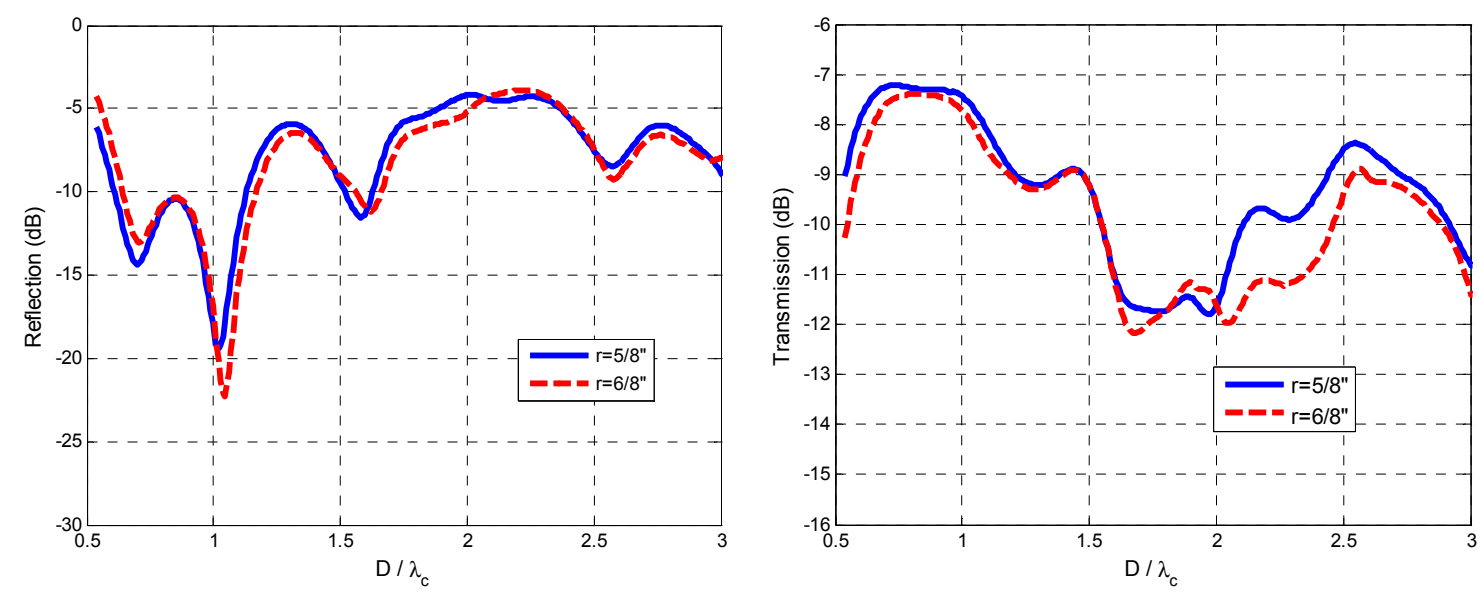

(b) Rebar diameter r.
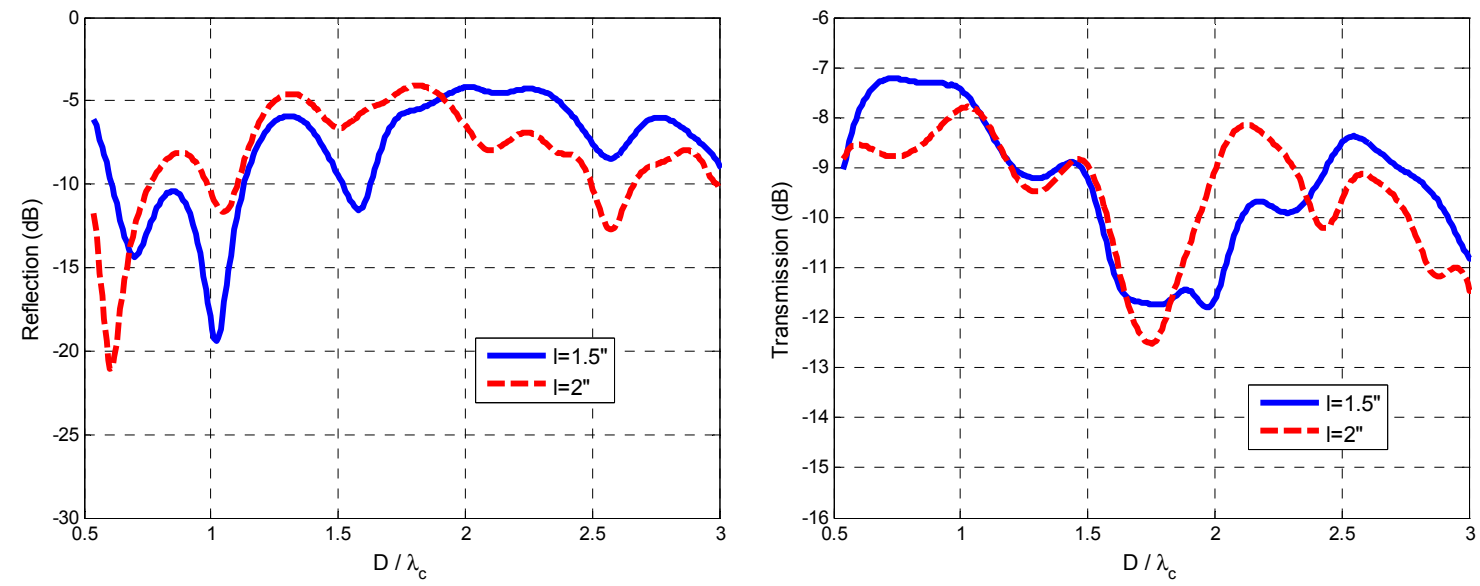

(c) Rebar depth 1.

Figure 4.17: Reflection and transmission coefficients for reinforced concrete slab with various rebar configurations. 
Furthermore, the rebar diameter $r$ is varied to $(6 / 8)$ " and the reflection and transmission coefficients of the reinforced concrete slab are shown in Figure 4.17(b) for two values of $r$, namely, (5/8)" and (6/8)". Results illustrate that the diameters of rebars has little effect on the wave propagation. In fact, the difference between the two cases is within $2 \mathrm{~dB}$ for the reflection coefficient.

The effects of the rebar layers depth $l$ inside concrete slab are also considered here. The reflection and transmission coefficients of reinforced concrete slab for two values of depth $l$ are shown in Figure 4.17(c). Our results illustrate that the variation of rebar depth may lead to shifting of the resonances occur at certain wavelengths. This is expected, since the variation of rebar depth inside concrete ma affect the combination effects of concrete slab and rebar layers on wave propagation. However, since typical value of rebar depth $l$ is within the range of 1.5 " and 2", the variation of rebar depth may not dramatically affect the wave propagation as variation of mesh period does.

Besides varying the geometrical parameters of $g, r$ and $l$, we also analyze the effects of different mesh configurations. We consider the case where the upper and lower layers of the rebar lattice are not perfectly parallel to each other, which happen often in practice. An offset between the rebar layers is shown in Figure 4.18, and it is described by the offset parameter $o$. Since the mesh period $g$ inside reinforced concrete slab varies from 4" to 12 ", it can lead to the variation of the offset parameter between 0 " and $6 "$. The effects of the offset on the reflection and transmission coefficients for reinforced concrete slab are shown in Figure 4.19, for offset of $0 \mathrm{~cm}$ and $3 \mathrm{~cm}$. The mesh period is fixed to 6" for both cases. Figure 4.19 illustrates that the offset of the lower mesh layer has minor effects on the wave propagation. This is expected, since the upper mesh layer would be 
firstly reached by the incident wave, and it primarily affects the propagation of EM waves through the reinforced concrete slab. Especially, when waves are shielded by the upper layer, waves with very small intensity will reach the lower layer. Therefore, in such case the effects of lower layer can be ignored. However, when waves penetrate efficiently through the first rebar layer, waves with increased intensity reach the lower layer, but the co-resonance of both layers is disturbed by the existence of a misalignment offset between the two rebar layers, thereby leading to smaller transmission coefficient. This phenomenon is more obvious for waves with shorter wavelengths, since they are more sensitive to the variation of geometrical configurations of rebar layers.

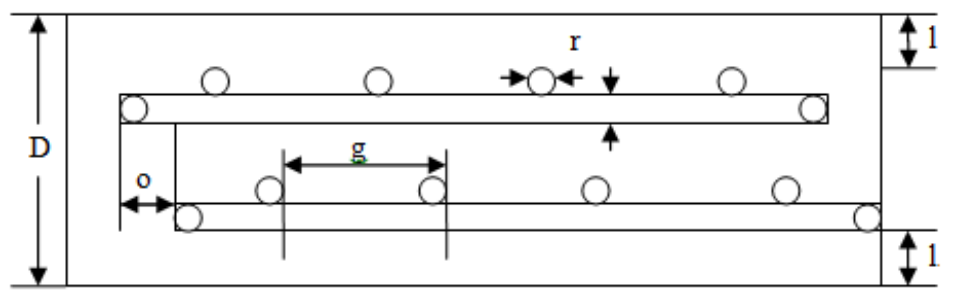

Figure 4.18: Offset between the two mesh layers inside reinforced concrete slab.

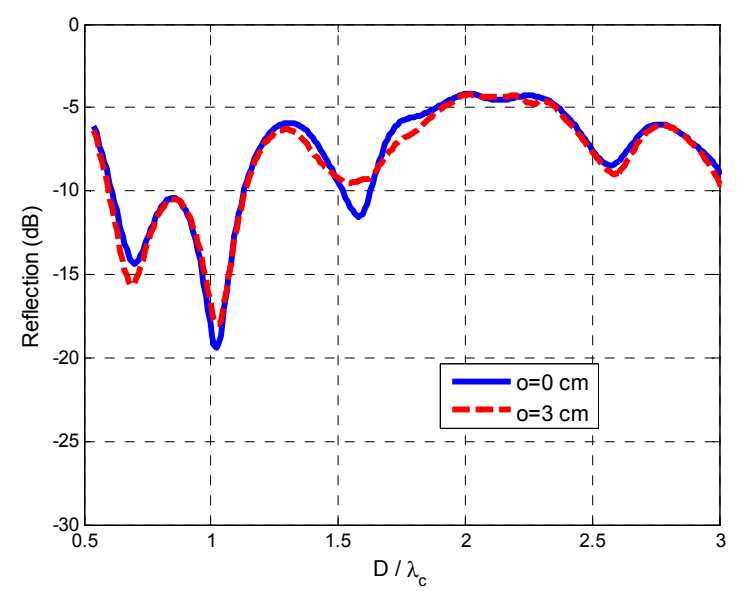

(a) Reflection.

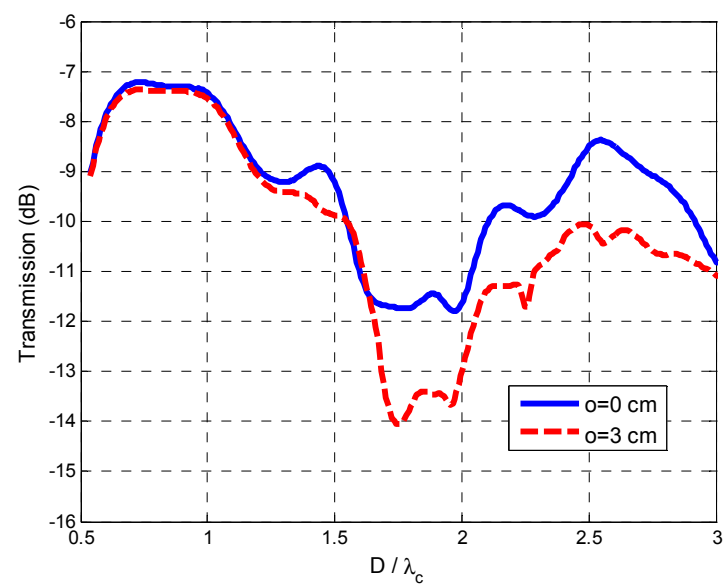

(b) Transmission.

Figure 4.19: Reflection and transmission coefficients for reinforced concrete slab with offset of rebar layers ( $\mathrm{g}=6$ " and $\mathrm{h}=6.2 \%)$. 
It could be concluded that the mesh period, $g$, dominates the wave propagation, since it determines the resonances of wave penetrating through mesh layers. Furthermore, the maximum electric field induced inside the reinforced concrete slab is calculated according to the conditions of Figure 4.17 and Figure 4.19, and the results are shown in Figure 4.20.

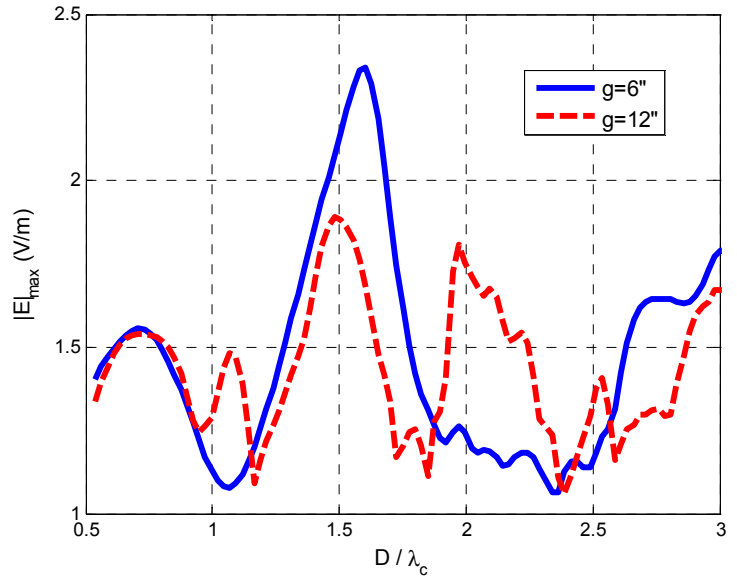

(a) Rebar period g.

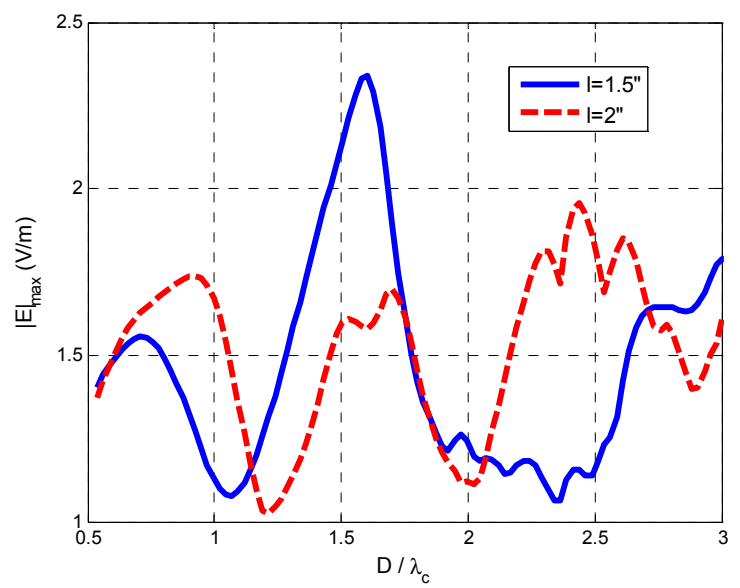

(c) Rebar depth 1 .

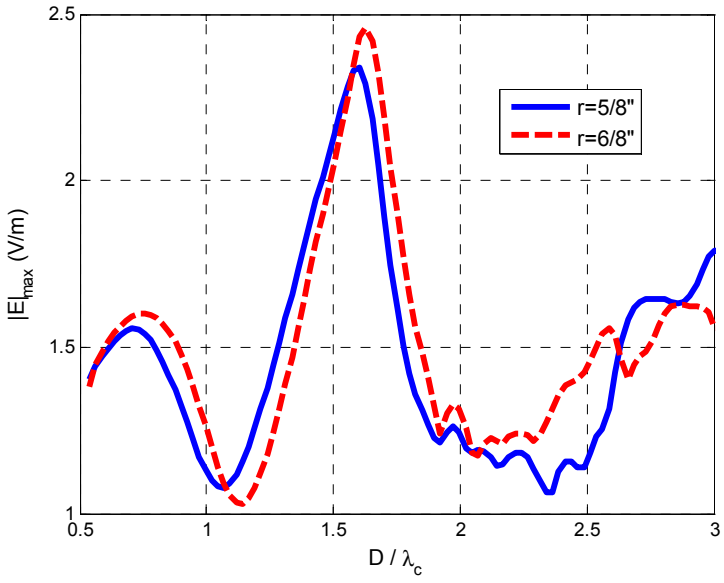

(b) Rebar diameter $r$.

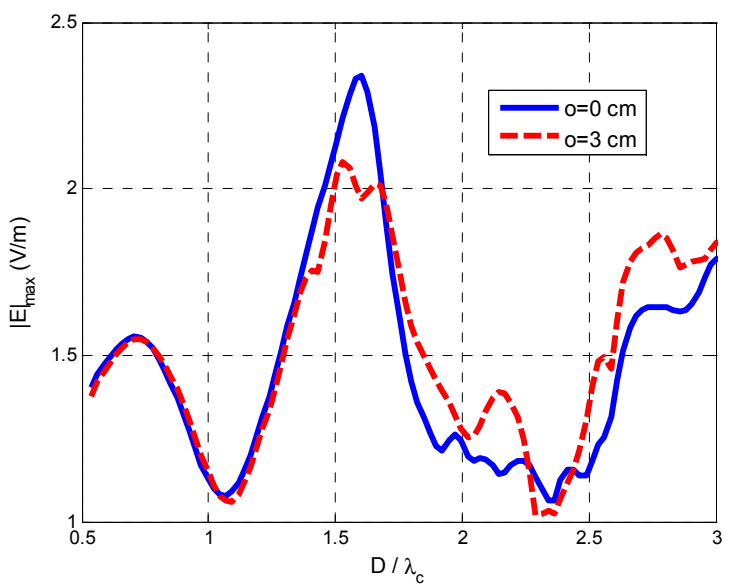

(d) Mesh layers offset 0 .

Figure 4.20: Maximum electric field induced inside reinforced concrete slab for various rebar and mesh configurations.

Results of Figure 4.20(a) and (c) illustrate that varying mesh period and rebar depth may impact the electric field induced inside reinforced concrete slab. For example, 
when mesh period $g$ is varied to 12 ", the peak value of electric field is reduced [see Figure 4.20(a)], since there is no co-resonance of rebar layers and concrete slab. The variation of induced maximum electric field corresponds to the variation of reflection and transmission coefficients of the reinforced concrete slab (see Figure 4.17 and 4.19).

Furthermore, results of Figure 4.20(b) and (d) illustrate that varying the rebar diameter and the offset between rebar layers have little effects on the electric field induced inside the reinforced concrete slab.

\subsection{Summary}

Full wave electromagnetic simulations are used in this chapter to analyze the EM power transmission through plain concrete slabs, rebar layers and reinforced concrete slabs. The slab's thickness and humidity conditions, as well as the rebar mesh configuration parameters, including mesh period, rebar diameter, mesh depth inside concrete and offset between the two layers were considered. Our results illustrated that the slab's thickness, humidity condition and mesh period of the rebar layers are the dominant parameters that control the EM wave propagation through reinforced concrete slabs. Also, antennas operating at 902-928 MHz ISM range were proven to be suitable for power transmission, since their performances are not sensitive to changes of the geometrical configurations of the reinforced concrete slab. Also, such antennas should be buried between the two rebar layers, since more power can be received at these positions. 


\section{CHAPTER 5}

\section{RECTENNA DESIGN}

In previous chapters, the optimum conditions for wireless power transmission through plain and reinforced concrete are investigated, including operational frequencies, position inside concrete, incidence angle, polarization, etc. In this chapter, some practical considerations for embedded sensor power transmission and harvesting systems are discussed. Specifically, antenna coupling is analyzed in order to validate our findings. Also, designs of rectification circuits for our antenna are developed in order to convert the received electromagnetic power to DC power and charge the batteries of SHM sensors.

\subsection{Plain Concrete}

In Chapter 3 plane wave models were used to examine the power loss level of wireless power transmission through plain concrete. In this section, optimum conditions identified by the plane wave model will be validated through antenna coupling simulations. Also, optimum rectenna designs will be developed for powering sensors

embedded in concrete. All simulations are performed with either Ansoft Designer or Ansoft HFSS. Also, all simulations use the extended Debye model for concrete permittivity [60].

\subsubsection{Antenna Type}

In order to implement a wireless powering system and maximize the power transmission between a transmitting antenna in air and a receiving antenna buried inside 
concrete, a proper antenna type should be used. Various antennas are presented in [61] and [81] as wire antennas, aperture antennas, microstrip antennas, reflector antennas, array antennas, etc. Each antenna type can take different forms to achieve the desired radiation characteristics for a particular application. Since low cost, directional and compact antennas are highly desired for power transmission through reinforced concrete structure, wire and stripline antennas are very well suited for such applications.

In our scenario, two typical antennas (dipoles or patches) are analyzed in order to determine the proper antenna type for wireless power transmission through concrete slabs. Initially, two half-wavelength dipole antennas (one residing is air and the other one residing inside concrete slab) are designed to resonate at $915 \mathrm{MHz}$. The experimental setup is shown in Figure 5.1. The first dipole is one wavelength away from the interface in the air, and the second one is embedded at a depth $d$ of 4" inside a typical concrete slab, which has a thickness $D$ of 8 ". Both dipoles are also tuned to be resonant at $915 \mathrm{MHz}$.

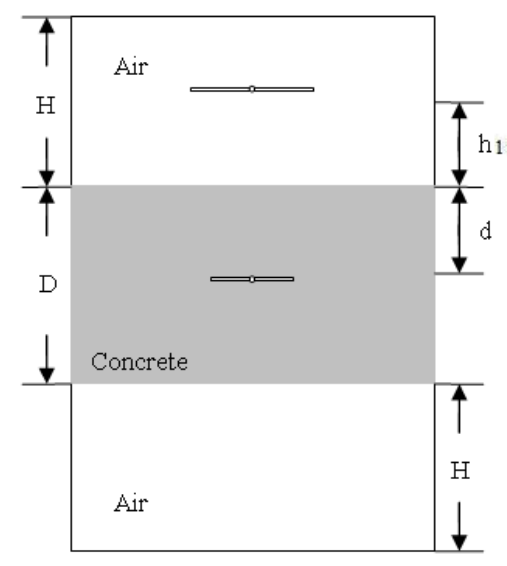

Figure 5.1: Simulation setup for two dipoles coupling in air-concrete slab model.

The return loss and coupling of the two dipoles are shown in Figure 5.2 for three cases: (a) free space (without the concrete slab in Figure 5.1), (b) dry concrete $(0.2 \%$ humidity) and (c) saturated concrete (6.2\% humidity). 


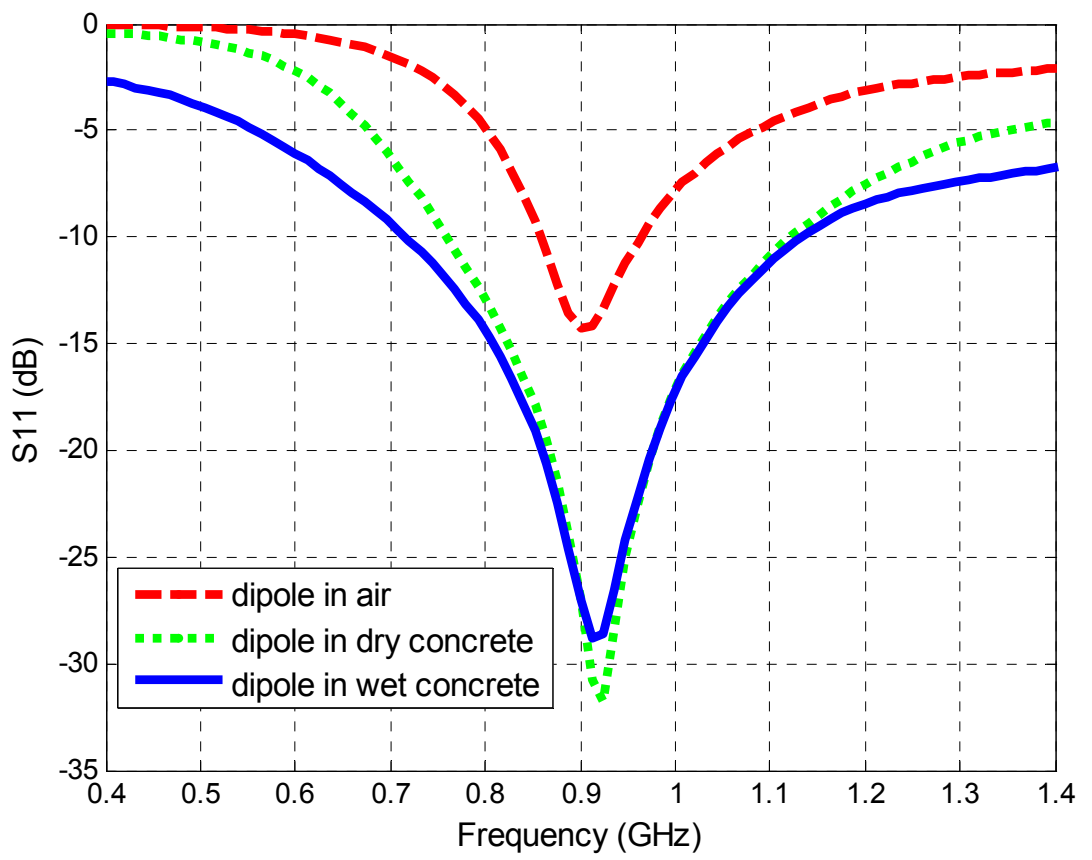

(a) Return loss.

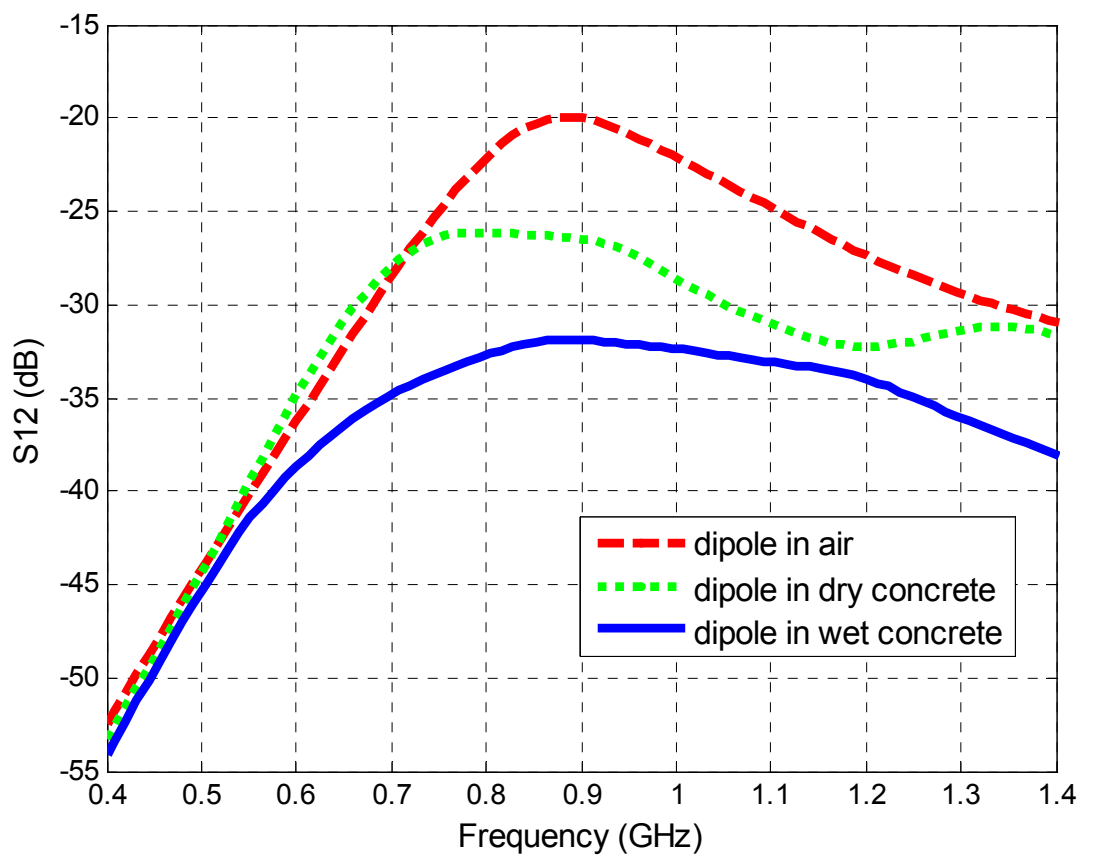

(b) Transmission loss.

Figure 5.2: Computed S-parameters of two dipoles coupling in free space, dry concrete and wet concrete.

In Figure 5.2(a), the $-10 \mathrm{~dB}$ return loss bandwidth for the antenna in air, dry concrete and wet concrete is $10.5 \%, 38.0 \%$ and $44.6 \%$, respectively. The dipole in the 
wet concrete has the widest bandwidth, which is due to the larger conductivity of the embedded media [80]. It can be seen from Figure 5.2(a) that a dipoles' performance is dramatically affected when it is embedded in the concrete. The coupling between the two dipoles is also analyzed and shown in Figure 5.2(b). It is seen that as the conductivity increases as the media changes from air to dry and wet concrete, the transmission loss between two dipoles also increases. For example, as shown in Figure 5.2(b), the coupling between two dipoles in air is approximately $6 \mathrm{~dB}$ larger than the coupling between two dipoles in air-to-dry concrete slab. This reduction is caused by the reflection of the electromagnetic wave at the air-to-concrete interface, the propagation loss in the dry concrete, and the decreasing gain of the dipole in the concrete.

Also, two pairs of probe-fed patch antennas (one for air and one for concrete) are designed at $915 \mathrm{MHz}$. Each patch is designed on a $\lambda_{0} / 100$ thick Rogers TMM $4\left(\varepsilon_{r}=4.5\right)$ substrate and fed by a coaxial probe [81]. Two patches are designed at $915 \mathrm{MHz}$ with length of $0.49 \lambda_{0} / \sqrt{\varepsilon_{r}}$ in air and $0.46 \lambda_{0} / \sqrt{\varepsilon_{r}}$ in concrete. The geometry configuration of the patch antennas is shown in Figure 5.3.

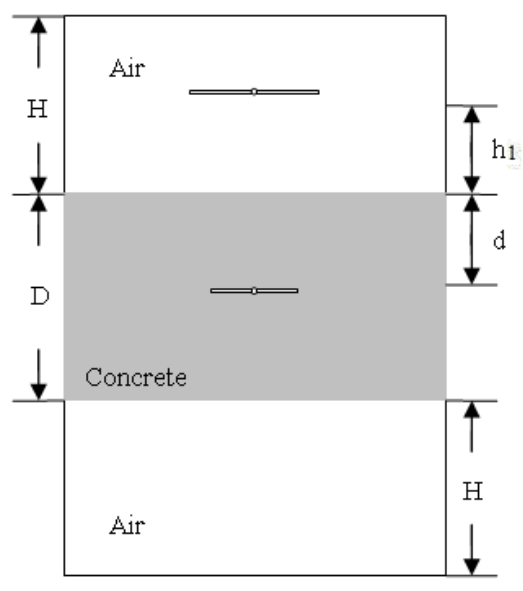

Figure 5.3: Simulation setup for two patches coupling in air-concrete slab model. 


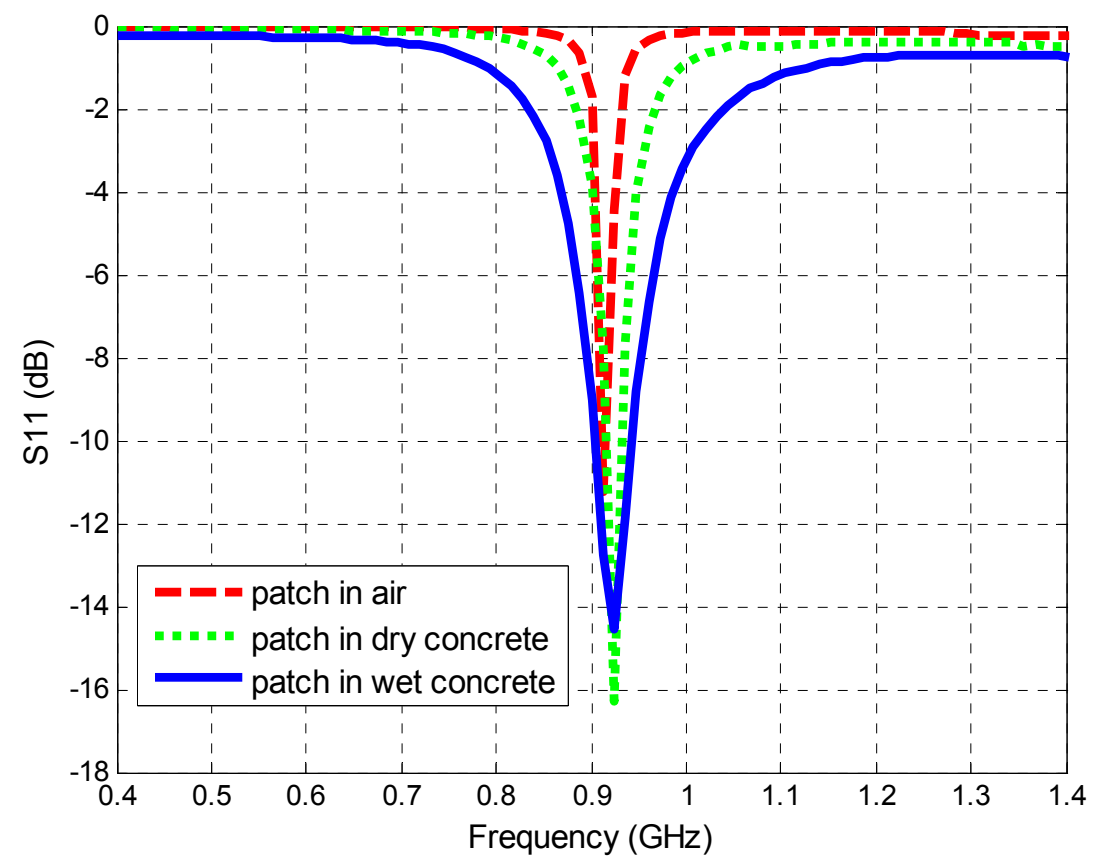

(a) Return loss.

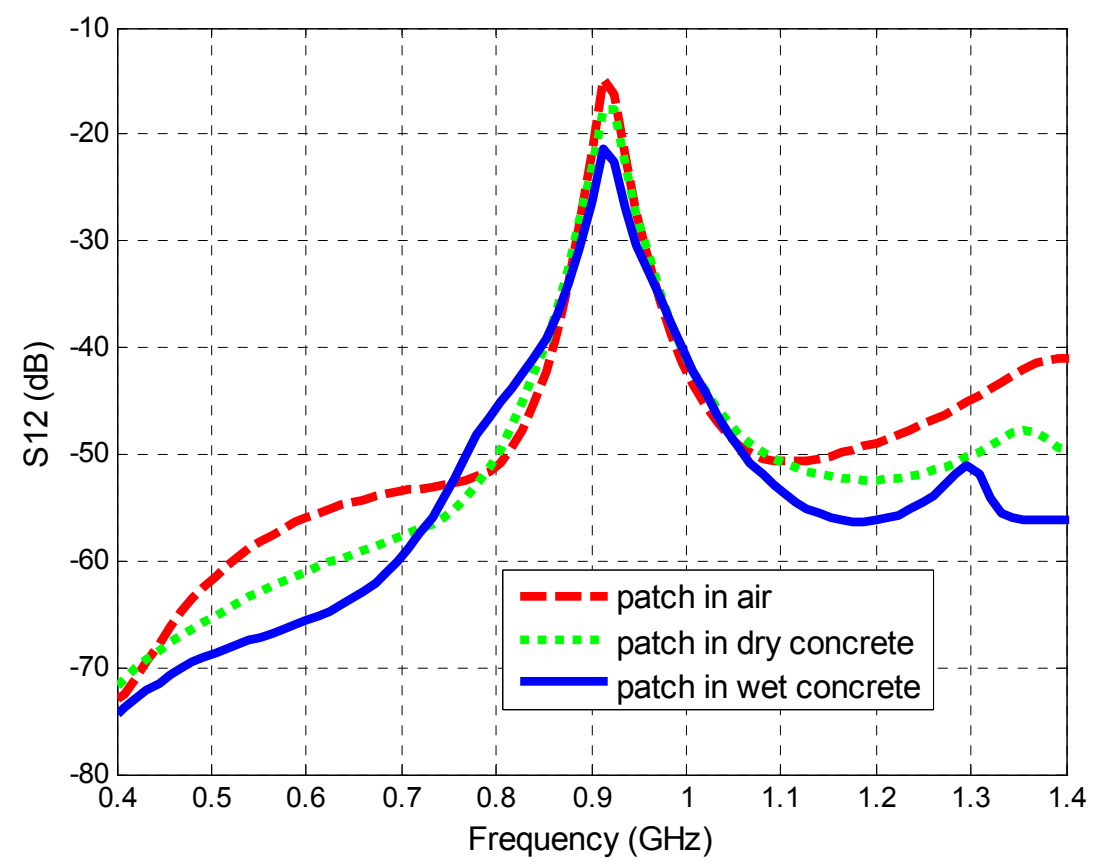

(b) Transmission loss.

Figure 5.4: Computed S-parameters of two patches coupling in free space, dry concrete and wet concrete. 
The return loss and transmission loss are plotted in Figure 5.4. Our results show that the patch antenna's performance is less sensitive to changes of concrete's material than the dipole's performance. For example, in Figure 5.4(a), the $-10 \mathrm{~dB}$ return loss bandwidth for the patch in air, dry concrete $(0.2 \%$ humidity) and wet concrete $(6.2 \%$ humidity) is $0.22 \%, 1.91 \%$ and $4.37 \%$, respectively. The differences between them are much smaller than the corresponding values obtained in our dipole analysis. The patch antenna is less sensitive than dipole because it is already on a substrate. Also, the patch is on a substrate with $\varepsilon_{r}=4.5$, which is similar to the permittivity of the dry concrete. Therefore, when the patch antenna is embedded into concrete it is essentially covered by a lossy dielectric [82] which causes the resonant frequency to decrease as the concrete's thickness above it increases. Finally, the half-power bandwidth only changes slightly when the thickness of covering material increases [83].

Furthermore, the coupling between the patches is plotted in Figure 5.4(b) for air, dry and saturate concrete. Figure 5.4(b) illustrates that the coupling between patches is greater than the one between the dipoles. For example, coupling between patches in air and wet concrete slab is about $10 \mathrm{~dB}$ larger than the one between the dipoles. When wirelessly powered sensors are embedded in concrete, the coupling between the sensor antennas should be maximized in order to achieve optimum performance. Therefore, our coupling results illustrate that patch antennas are more suitable for such applications.

\subsubsection{Patches Coupling}

In Chapter 3 we identified an optimum frequency of $20-80 \mathrm{MHz}$ corresponding to minimum transmission losses for power transmission through plain concrete. In this 
section, patch antenna analysis is performed in order to validate and confirm this important finding. Specifically, the coupling between two probe-fed patch antennas is analyzed using Ansoft Designer. One patch resides in air with length of $0.49 \lambda_{0} / \sqrt{\varepsilon_{r}}$, and the other patch resides inside concrete is tuned to be resonant at the same frequency, as shown in Figure 5.5. $\lambda_{0}$ is the wavelength in free space and $\varepsilon_{r}$ is the relative permittivity of concrete. In Figure 5.5, thickness of the air and concrete, $H$, is $4 \mathrm{~m}$. Patch in the air is one wavelength $\left(\lambda_{0}\right)$ away from the air-to-concrete interface at the patch resonant frequency, and the patch buried inside concrete is buried at a depth, $d$, of $0.1032 \mathrm{~m}$.

Figure 5.6 illustrates the results of our analysis where the frequency range is normalized with the resonant frequency of the patch antenna (patch in the air and the one in concrete). Specifically, Figure 5.6 illustrates the coupling $\left(\mathrm{S}_{12}\right)$ difference between various humidity conditions and the dry concrete (humidity $0.2 \%$ ) for a resonant frequency of $1 \mathrm{GHz}$. It should be pointed out that in our analysis we need to maximize the coupling (minimize the power loss) between the two patches in order to obtain optimized wireless communication between the two antennas. Also, we need to maximize the wireless power transfer between the two patches in order to charge the battery of embedded sensors inside the concrete. As expected, the power loss for higher humidity conditions is larger than the one for low humidity conditions, as shown in Figure 5.6. For example, the coupling of two patches for $12 \%$ humidity condition is approximately 8.5 $\mathrm{dB}$ smaller than the coupling of two patches for dry concrete (i.e., $0.2 \%$ humidity). The plane wave analysis predicts a $5.9 \mathrm{~dB}$ difference between the same corresponding humidity conditions, which is shown in Figure 3.6. The difference between plane wave analysis and patches' simulation results is due to the pattern and gain of the patch 
antennas. Therefore, in order to design a transmitting-receiving power system for the airto-plain concrete interface and maximize the power transfer between the two patches, we must take into account the humidity conditions of the concrete since they have a significant and detrimental effect to the coupling between the two patches.

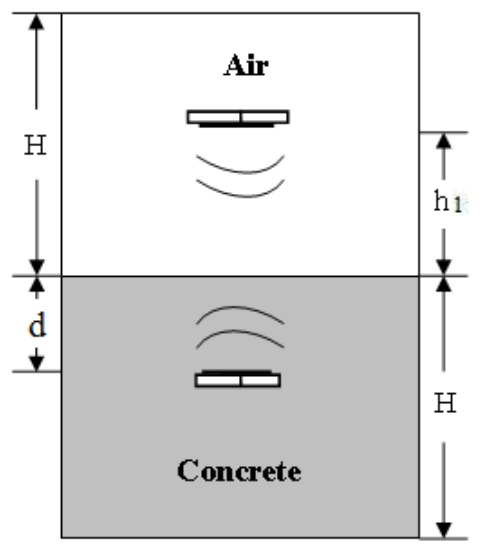

Figure 5.5: Simulation setup of two patches in the air-concrete model.

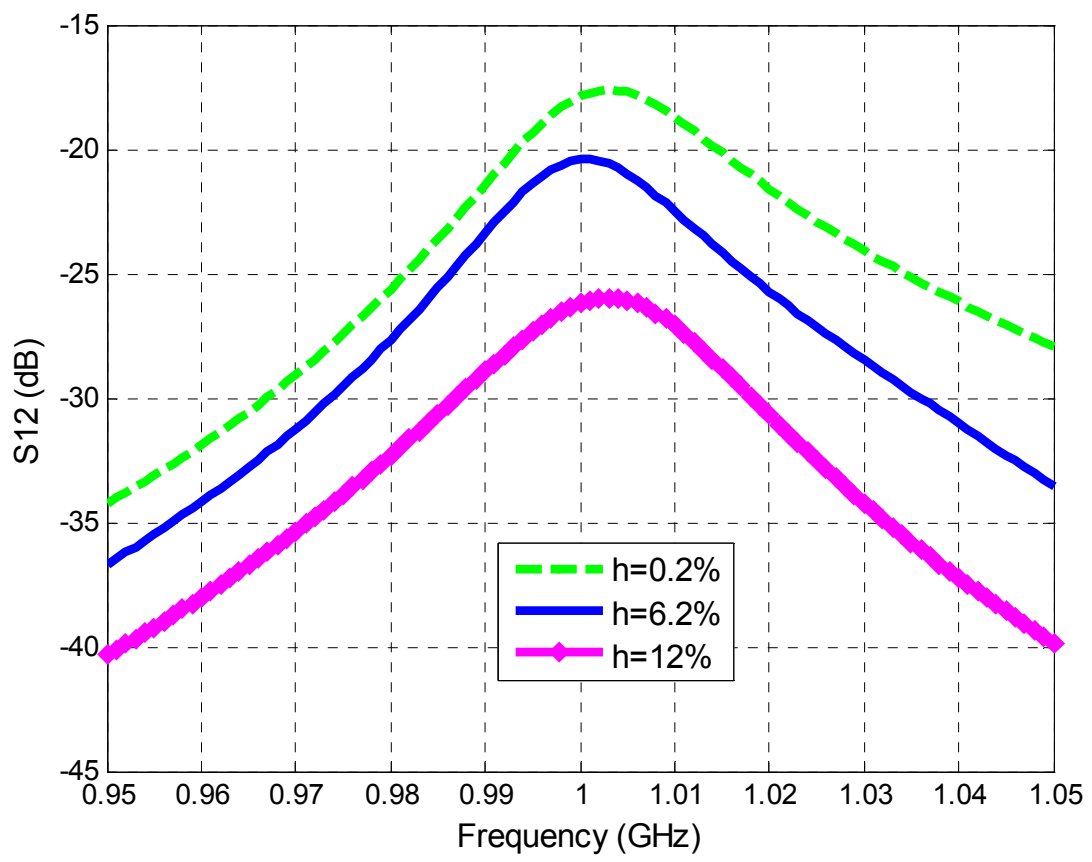

Figure 5.6: Patches coupling for different concrete humidity conditions. 
Also, a pair of patches (one in air and one residing inside the concrete, see Figure 5.5) with matching resonant frequencies is designed for various resonant frequencies, i.e., $70 \mathrm{MHz}, 200 \mathrm{MHz}$ and $1 \mathrm{GHz}$, etc. This was done because $70 \mathrm{MHz}$ resides in the optimum operation frequency range as per the results of Chapter 3. It can be seen from Figure 5.7 that the coupling of the patches resonating at $70 \mathrm{MHz}$ is larger than the coupling of the patches with other resonant frequencies. For example, the coupling of the two patches resonating at $1 \mathrm{GHz}$ is about $2.5 \mathrm{~dB}$ smaller than the coupling of the patches resonating at $70 \mathrm{MHz}$. This indeed validates our findings in the previous sections and indicates the existence of an optimum frequency range at 20-80 MHz.

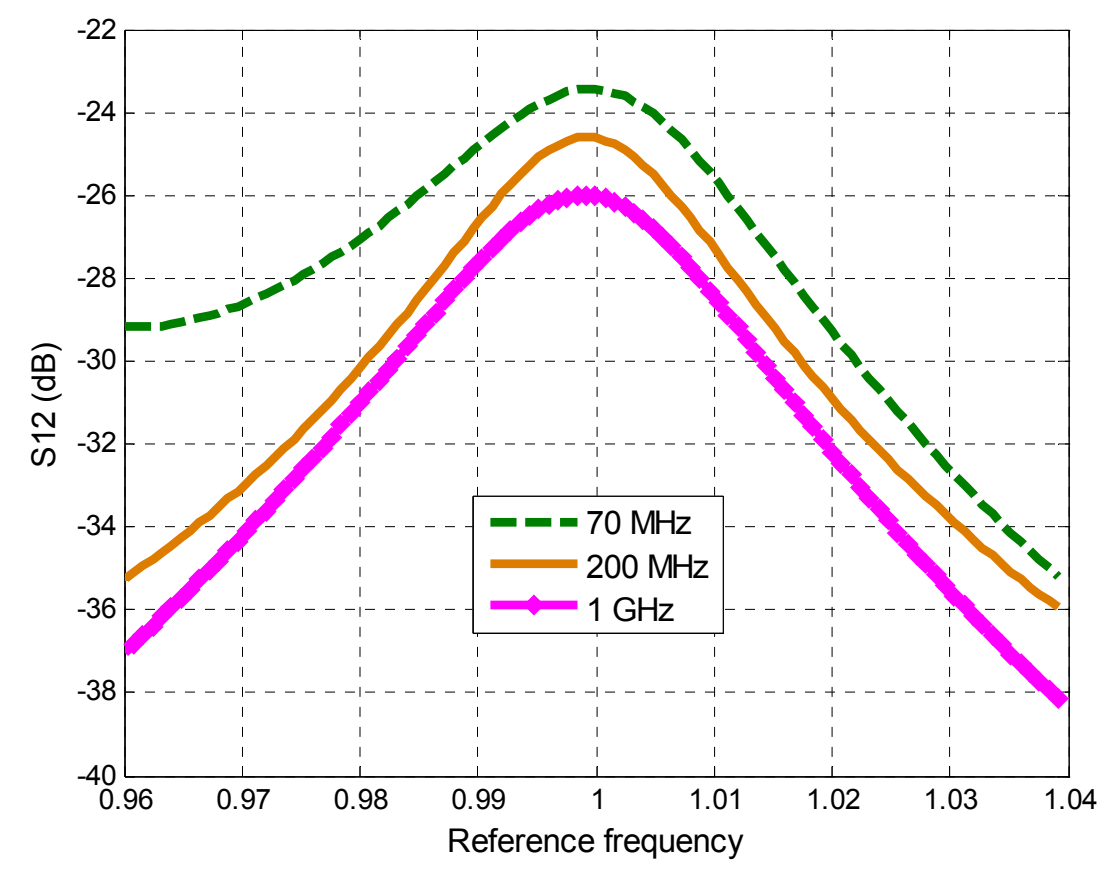

Figure 5.7: Patches coupling for different resonant frequencies.

\subsubsection{Rectification Circuit}

In order to charge the sensor's rechargeable battery, a rectifying circuit must be connected to the receiving antenna to achieve the RF to DC power transmission. 
Rectifying circuits are similar to AC/DC converters; such AC/DC converter circuits are complex and include protective circuitry to reduce noise. However, such complex circuit may require more power than what is available in the case of embedded sensors. Therefore, simpler rectification circuit designs are necessary for SHM sensors.

The simplest rectify circuit design is a peak detector, which requires only a diode and a capacitor to function [84]. As shown in Figure 5.8(a), on the positive half of the AC wave, the diode turns on and current flows to charge the capacitor. While on the negative half of the AC wave, the diode turns off, and the voltage in the capacitor remains the peak of the AC wave. When a load is added, the output voltage decreases during the negative cycle of the AC input, and the rate of decrease is determined by the resistance of the load and the capacitance of the capacitor. This type of rectifying circuit is widely used for semi-passive RFID tags. Because only one half of the input waves are rectified, peak detector is inefficient for power conversion. In order to improve the efficiency, full-wave rectification solutions, which can convert both polarities of the input waves to DC, were developed. Voltage doubler is a typical full-wave rectification circuit, as shown in Figure 5.8(b), where its output voltage is expected to be twice that of the input. In the positive half of the AC, current is rectified by $D_{2}$ and $C_{2}$, while in the negative half by $D_{1}$ and $C_{l}$. During the positive cycle, the voltage stored on $C_{l}$ from the negative cycle is transferred to $C_{2}$. Therefore, $C_{2}$ is roughly twice the peak voltage of the power source minus the turnon voltage of the diode. When connecting voltage doubler stages in series, as shown in Figure 5.8(c), the output is an AC signal with a DC offset. The number of stages is directly proportional to the amount of voltage obtained at the output of the system. 


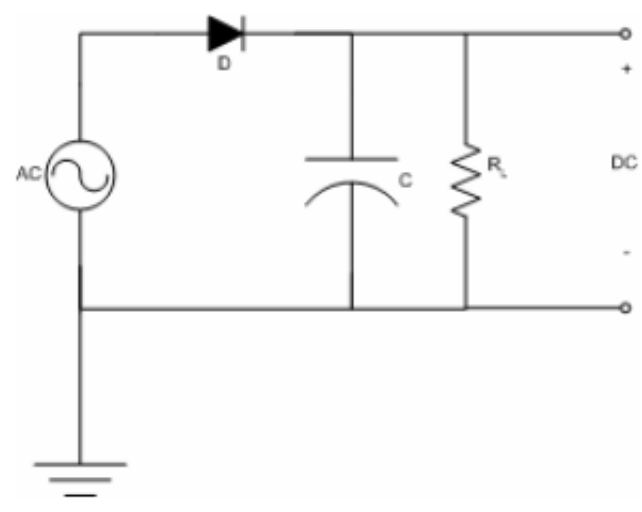

(a) Peak detector.

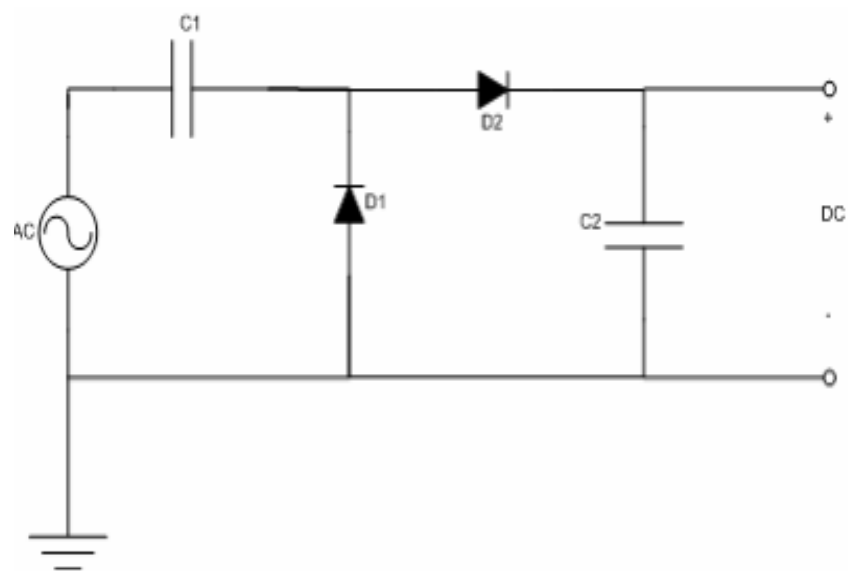

(b) Voltage doubler.

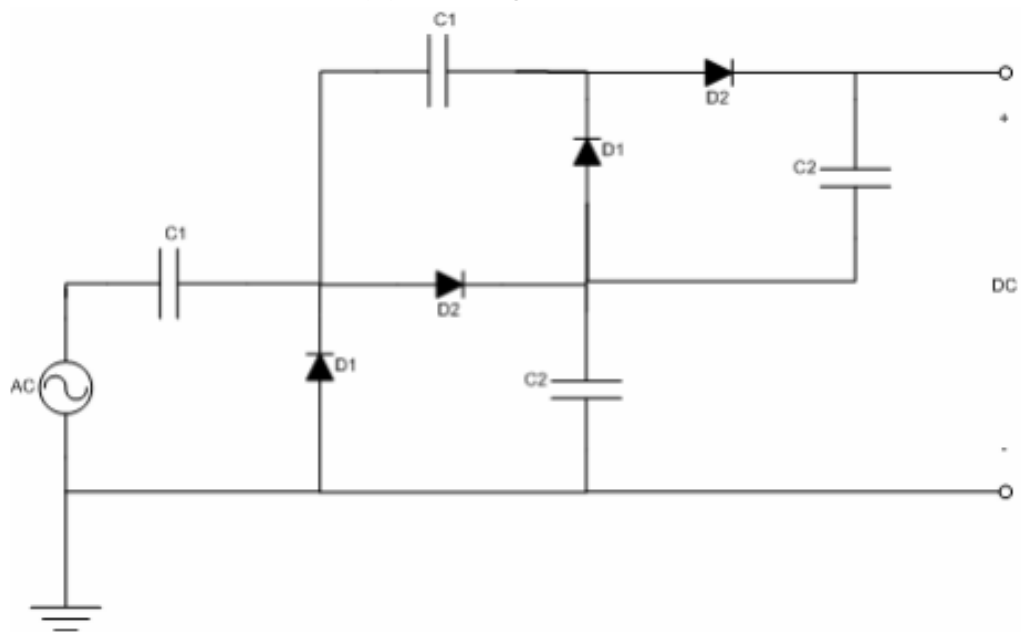

(c) Stages of voltage doubler.

Figure 5.8: Rectification circuit types. 
To optimize the rectification efficiency, three types of rectifying circuits are used for comparison, and the analysis is performed in Ansoft Nexxim. Diodes are the main elements of a rectification circuit, and the Agilent HSMS-282x series Schottky diodes are chosen for our design. HSMS-282x series are used for detector applications with input power levels greater than $-20 \mathrm{dBm}$, and frequencies below $4.0 \mathrm{GHz}$ [85], which meet the requirements of the sensor charging system.

The modeling parameters for HSMS-282x diodes are given by Agilent data sheets, and the SPICE parameters are shown in Table 5.1. SPICE parameters can also be applied in Ansoft Nexxim for simulation modeling since both of them perform simulation using Netlists [86]. In Table 5.1, $R_{S}$ is the series resistance and $C_{J 0}$ is the junction capacitance. These two factors have the most effect on the diode by determining the turn-on voltage and rise time. Lower series resistance leads to lower voltage needed to turn on the diode, and lower junction capacitance raises voltage faster. $B_{V}$ is the reverse breakdown voltage, $E_{G}$ presents the band-gap energy. $I_{B V}$ is the current breakdown voltage, and $I_{S}$ is the saturation current. $N$ is the emission coefficient, while $M$ is the grading coefficient. These parameters are used in our simulation setups in Nexxim.

Table 5.1: HSMS-282x SPICE parameters.

\begin{tabular}{c|c|c} 
Parameter & Units & Value \\
\hline $\mathrm{B}_{\mathrm{V}}$ & $\mathrm{V}$ & 15 \\
$\mathrm{C}_{\mathrm{J} 0}$ & $\mathrm{pF}$ & 0.7 \\
$\mathrm{E}_{\mathrm{G}}$ & $\mathrm{eV}$ & 0.69 \\
$\mathrm{I}_{\mathrm{BV}}$ & $\mathrm{A}$ & $1 \mathrm{E}-4$ \\
$\mathrm{I}_{\mathrm{S}}$ & $\mathrm{A}$ & $2.2 \mathrm{E}-8$ \\
$\mathrm{~N}$ & no units & 1.08 \\
$\mathrm{R}_{\mathrm{S}}$ & $\Omega$ & 6.0 \\
$\mathrm{P}_{\mathrm{B}}$ & $\mathrm{V}$ & 0.65 \\
$\mathrm{P}_{\mathrm{T}}$ & no units & 2 \\
$\mathrm{M}$ & no units & 0.5
\end{tabular}




\subsubsection{Rectifier Design}

In order to charge the sensor battery, we must use a rectification circuit along with the antenna (rectenna) to convert the received electromagnetic power to DC power. Specifically, we simulate a wireless power transfer system consisting of two stripline antennas operating at $70 \mathrm{MHz}$, one is buried at a depth $d=4$ " inside concrete with $0.2 \%$ humidity, while the transmitting antenna is placed $2.14 \mathrm{~m}$ above the air-to-concrete interface, as shown in Figure 5.5. Then the equivalent two-port network of two patches coupling is exported from Ansoft Designer and imported to Ansoft Nexxim, which enables the co-simulation of circuits. In Nexxim, the patch antenna located in air is connected to a $1 \mathrm{~W}$ power source, and the one that is embedded in concrete is connected to a rectification circuit. Three rectification circuits mentioned in the previous section are calculated, including the peak detector, voltage doubler, and stages of voltage doubler. Our results illustrate that the voltage doubler is more suitable for our wireless powering systems, since it provides larger amount of power to the load. Our system setup is shown in Figure 5.9, where the power source is $1 \mathrm{~W}$ with $50 \mathrm{Ohm}$ internal resistance, two capacitors $C_{r 1}$ and $C_{r 2}$ are $6 \mathrm{nF}, D_{r 1}$ and $D_{r 2}$ are HSMS-2820 Schottky detector diodes, and the load is $100 \mathrm{Ohm}$. The power delivered to the load is $55.2 \mathrm{~mW}$, which is equivalent to a wireless power transfer efficiency of $5.52 \%$.

In order to optimize the efficiency of the wireless powering system we design matching circuits for the antennas and the rectifier. Figure 5.10 illustrates the complete circuit layout, where the two antennas are matched to $50 \mathrm{Ohm}$ using matching networks. Inductor $L_{l}$ is $200 \mathrm{nH}, L_{2}$ is $100 \mathrm{nH}, L_{m}$ is $300 \mathrm{nH}$, and capacitor $C_{l}$ is $50 \mathrm{pF}, C_{2}$ is $45 \mathrm{pF}$, $C_{m}$ is $6 \mathrm{nF}, C_{r 2}$ is $15 \mathrm{nF}$. Also, the rectification circuit is matched to $50 \mathrm{Ohms}$. The 
matched system provides an improved wireless power transfer efficiency of $6.4 \%$ corresponding to a delivered power to the load of $64 \mathrm{~mW}$.

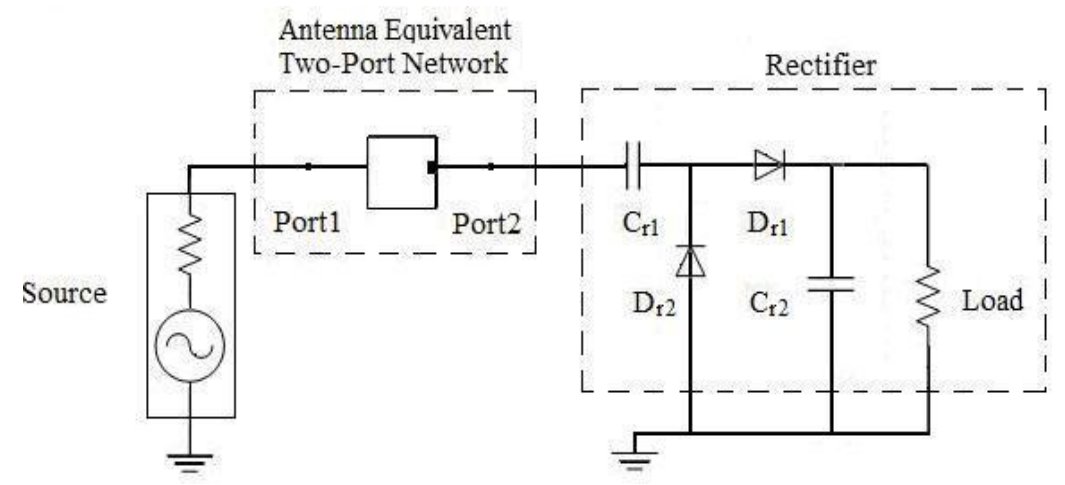

Figure 5.9: Wireless powering system setup with voltage doubler.

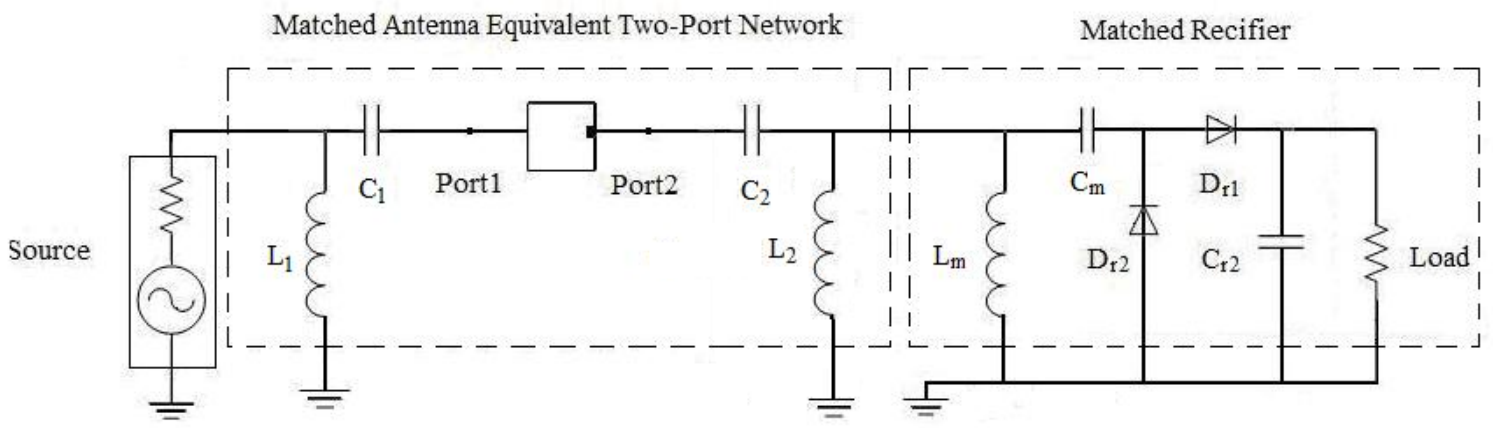

Figure 5.10: Wireless powering system setup with matched voltage doubler.

\subsubsection{Energy Budget}

In previous sections the wireless power transmission system for powering sensors embedded in reinforced concrete was designed. In this section, the energy budget for wireless sensors is analyzed, and the sensor battery charging times are calculated.

A common problem for energy harvesting devices is that the amount of energy harvested must be sufficient to power the electronic device of interest [87]. For our application, approximately a power of $50 \mathrm{~mW}$ is required to run commercial sensor nodes [88] and we will use a rechargeable battery as a power source. 
The $80 \mathrm{mAh}$ Nickel-metal hydride button cell battery [89] is specifically chosen to power the wireless sensor node embedded in concrete since it can withstand more harsh environments and it offers more energy per unit volume than the nickel-cadmium type [90]. It should be noted that neither a charge controller nor voltage regulator need to be incorporated into the circuit for this type of battery [89]. Also, the size of this battery is suitable for nodes embedded in the concrete.

Assuming that we use the same nodes as the ones described in [91], the rechargeable button cell battery consumes $1.25 \mathrm{mAh}$ per day and can work 64 days without charging while in sleep mode. In working days, this battery consumes $6.9 \mathrm{mAh}$ per day and can work for only 11 days with $5.8 \%$ duty cycle. This battery can be fully charged using our wireless powering system of Figure 5.10, which has $25.3 \mathrm{~mA}$ output current, in 3.2 hours.

\subsection{Reinforced Concrete}

An optimum frequency range of 902-928 MHz was identified in Chapter 4 for powering sensors embedded in reinforced concrete slabs. In this section, optimum rectenna will be designed for charging the batteries of embedded sensors in reinforced concrete. Both far field and near field coupling between patch antennas are considered and all simulations are performed with either Ansoft Designer or Ansoft Nexxim.

\subsubsection{Rectenna Design}

Based on the optimum conditions we have identified for minimizing the power transmission loss, a stripline antenna resonant at $915 \mathrm{MHz}$ is designed for receiving power inside reinforced concrete slab for the embedded sensors. Geometry of the antenna 
is shown in Figure 5.11, the dielectric layers on both sides of the radiator can protect it, and can also be used to adjust the resonant frequency. The superstrate above the radiator is Rogers TMM $4\left(\varepsilon_{\mathrm{r}}=4.5\right)$, which is close to the permittivity of dry concrete at $915 \mathrm{MHz}$, while the substrate is Rogers TMM $6\left(\varepsilon_{\mathrm{r}}=6\right)$, which is close to the permittivity of wet concrete at $915 \mathrm{MHz}$ [60]. This design aims at mitigating the effects of varying concrete humidity (from wet to dry) to the antenna's resonant frequency. The stripline's thickness is $6.56 \mathrm{~mm}$, and the radiator size is $62 \mathrm{~mm} \times 62 \mathrm{~mm}$. The transmitting antenna in the air is a probe-feed square patch antenna with the Rogers TMM 4 substrate, a thickness of $3.28 \mathrm{~mm}$ and a radiator size of $76 \mathrm{~mm} \times 76 \mathrm{~mm}$. For the setup in Figure 5.11, the air thickness, $H$, is $0.5 \mathrm{~m}$, while the concrete slab thickness, $D$, is $20.32 \mathrm{~cm}$. The patch antenna is placed at a height, $h_{1}$, of $0.3 \mathrm{~m}$ in the air, and the receiving stripline antenna is placed at a depth, $d_{1}$, of $8 \mathrm{~cm}$ inside concrete. The configuration of the rebars has a depth inside concrete, $l$, of $3.81 \mathrm{~cm}$, mesh period, $g$, of $15.24 \mathrm{~cm}$, and diameter of the rebar, $r$, of $1.59 \mathrm{~cm}$.

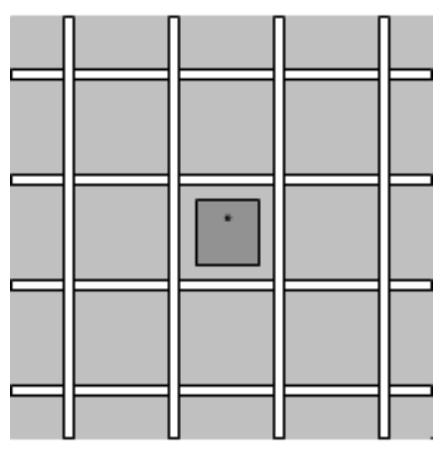

(a) Top view.

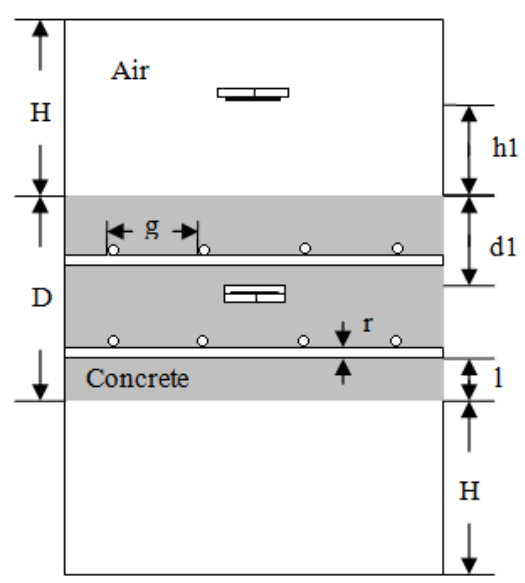

(b) Side view.

Figure 5.11: Simulation setup for two antennas in the air-reinforced concrete model. 


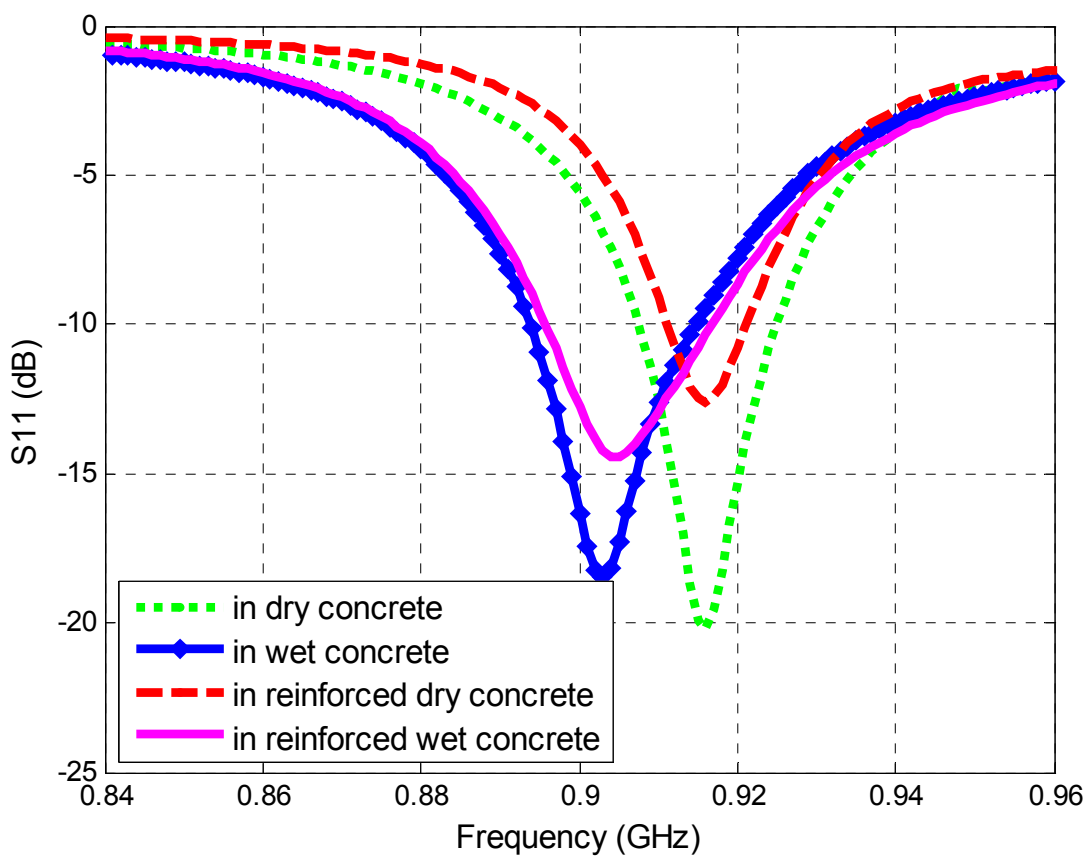

(a) Return loss.

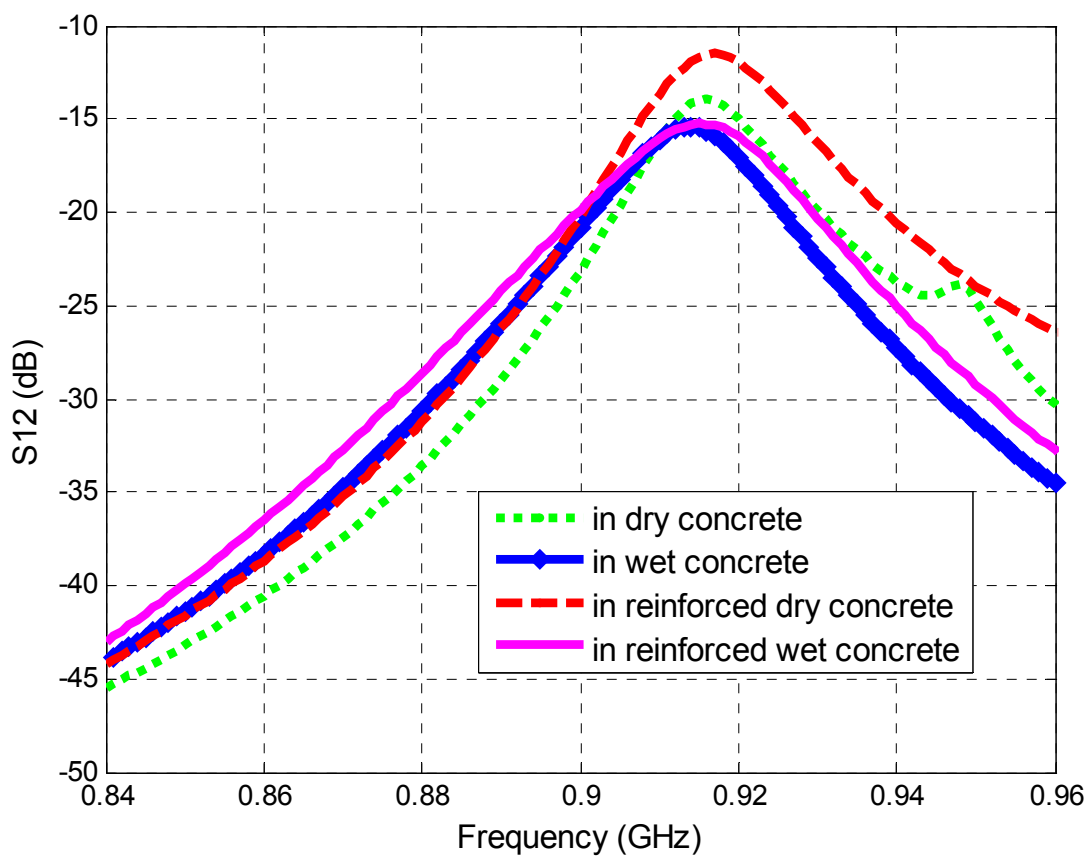

(b) Transmission loss.

Figure 5.12: Computed S-parameters of two antennas coupling.

The return loss and transmission loss for this antenna are plotted in Figure 5.12 for different concrete humidity conditions $(0.2 \%$ and $6.2 \%)$ and rebar configurations 
(with and without rebars). Figure 5.12(a) illustrates that the stripline antenna is resonant at $915 \mathrm{MHz}$ in the dry concrete slab. When the concrete is wet, the central resonant frequency shifts to $903 \mathrm{MHz}$. However, the $-10 \mathrm{~dB}$ return loss bandwidth still includes $915 \mathrm{MHz}$. Also, adding rebar grid has little effects on the central resonant frequency of the stripline antenna.

The coupling between the two antennas is shown in Figure 5.12(b). Figure 5.12(b) shows that the coupling for the wet concrete (6.2\% humidity) is approximately $4 \mathrm{~dB}$ less than the coupling for dry concrete $(0.2 \%$ humidity). When rebar grid is added, the coupling between the two antennas for both wet concrete and dry concrete conditions increased.

When adding the $1 \mathrm{~W}$ power source to the transmitting antenna and a voltage doubler to the receiving antenna, as shown in Figure 5.9 (two capacitors $\mathrm{C}_{\mathrm{r} 1}$ and $\mathrm{C}_{\mathrm{r} 2}$ are varied to $80 \mathrm{pF})$, the power delivered to the load for the reinforced dry concrete $(0.2 \%$ humidity) is $35.3 \mathrm{~mW}$, which is equivalent to a wireless power transfer efficiency of $3.53 \%$. The power delivered to the load for the reinforced wet concrete ( $6.2 \%$ humidity) is $13.2 \mathrm{~mW}$, which is equivalent to a wireless power transfer efficiency of $1.32 \%$.

\subsubsection{Near Field Coupling Solutions}

In previous sections, we concluded that patch antennas are more suitable for wireless power transmission, since they provide significantly smaller transmission loss (i.e., larger coupling) than dipoles. In this section, the performance of patch antennas in near field coupling is analyzed at three typical ISM frequencies (i.e., $433 \mathrm{MHz}, 915 \mathrm{MHz}$, and $2.45 \mathrm{GHz}$, etc.), and the effects of three typical configurations of rebar layers (i.e., 
cross bars, bonded-junction bars and vertical bars) are considered. This study is performed in order to determine the optimum antenna operational frequency for maximum wireless power transmission from air to reinforced concrete structures in near field, where the transmitting antenna is touching the surface of the concrete slab (i.e., external antenna touches the reinforced concrete wall for powering and communicating with embedded SHM sensors, etc.).

Two pairs of probe-fed patch antennas (one for air and one for reinforced concrete slab) are designed for three typical ISM resonant frequencies and the geometrical setup is shown in Figure 5.13. The transmitting antenna in air is touching the air-to-concrete surface as we assume that the transmitting antenna will be installed on the outside surface of the reinforced concrete slab. The receiving antenna is installed between the two mesh layers. Three typical rebar configurations are considered: vertical bars, bonded-junction bars and cross bars, as shown in Figure 5.13. The polarization of patches is parallel to the direction of vertical bars in Figure 5.13(a). Also, for cross bars in Figure 5.13(c), the polarization of patches is parallel to the direction of longitudinal bars. This is done to increase the power transmission efficiency because vertical bars and longitudinal bars perform significant shielding for the power transmission.

For the simulation setup, same configuration parameters are chosen for these three cases. Specifically, the patch antenna is buried at $9 \mathrm{~cm}$ depth $d$ inside the concrete slab, the rebar diameter, $r$, is $(5 / 8)$ " and the mesh depth, $l$, is 1.5 ". Since the mesh period, $g$, dominates the impact of rebar layers on the antenna performance, only the mesh period is varied (4"-12") in our simulations. 

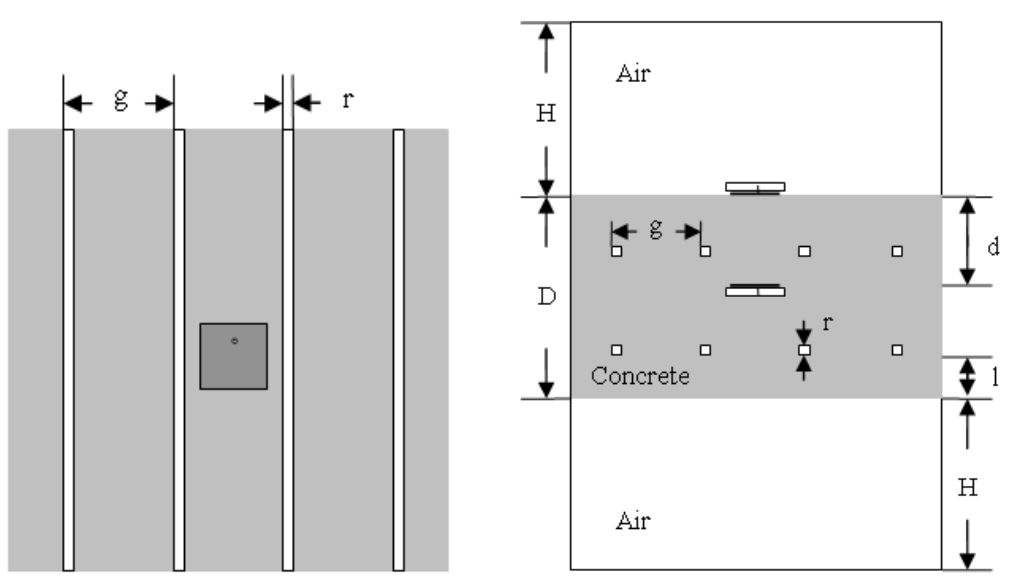

(a) Vertical bars.
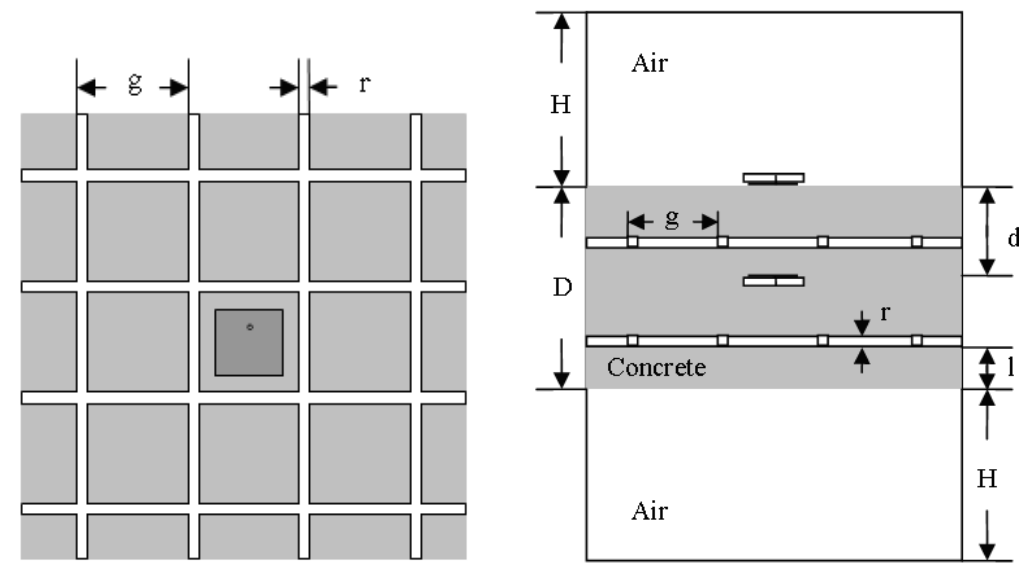

(b) Bonded-junction bars.
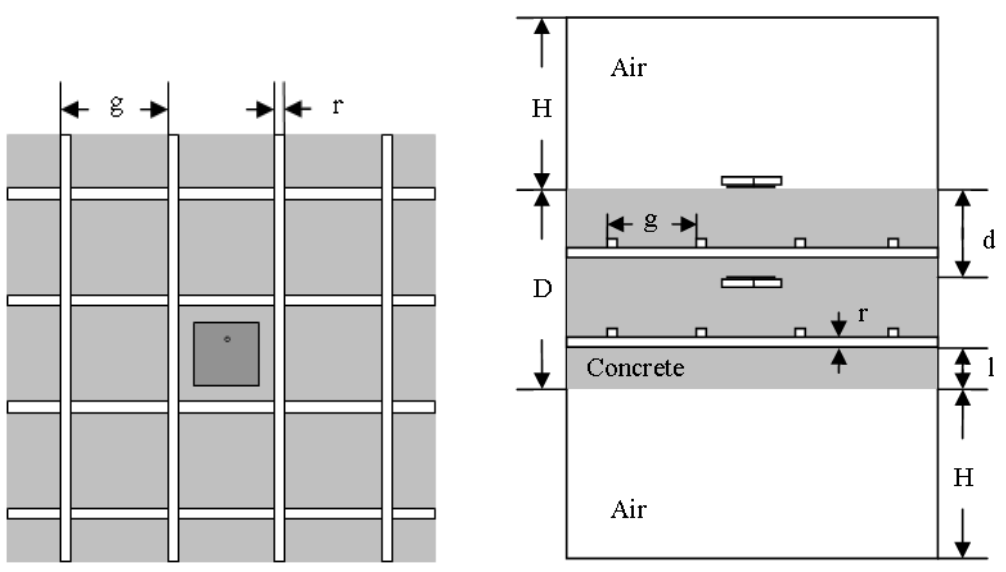

(c) Cross bars.

Figure 5.13: Simulation setup for three rebar types. 
Figure 5.14 illustrates the effects of rebar layers on the coupling between patch antennas at three frequencies, and the mesh period is normalized with the wavelength inside concrete. The coupling between antennas working at $433 \mathrm{MHz}$ is shown in Figure 5.14 (a). These results illustrate that when mesh period is larger than the wavelength (32.44 $\mathrm{cm}$ inside concrete), all these three types of rebar configurations have limited effects on antennas' performance and antennas' coupling. However, when the mesh period is smaller than one wavelength, especially when it is less than 0.6 times the wavelength, the antenna coupling is dramatically reduced. For example, as shown in Figure 5.14 (a), the coupling at $\mathrm{g} / \lambda_{\mathrm{c}}$ of 0.6 (corresponds to $\mathrm{g}=7.7$ ") is approximately 13 $\mathrm{dB}$ less than the corresponding value for plain concrete slab case. Similar trend can also be observed for antennas coupling at $915 \mathrm{MHz}$ and $2.45 \mathrm{GHz}$, as shown in Figure 5.14(b) and (c). However, since $\lambda_{c}$ for $915 \mathrm{MHz}$ and $2.45 \mathrm{GHz}$ are smaller $(15.42 \mathrm{~cm}$ and $5.77 \mathrm{~cm}$, respectively), they would not be dramatically shielded by rebars with the smallest mesh period $(10.16 \mathrm{~cm})$, and are more suitable for power transmission.

Furthermore, Figure 5.14(b) and (c) illustrate that different rebar types have similar impacts on patches coupling. Also, $915 \mathrm{MHz}$ is more suitable for powering sensors embedded in reinforced concrete slab through near field coupling method, since antenna coupling at $915 \mathrm{MHz}$ is stronger than at $2.45 \mathrm{GHz}$. For example, the average level for antenna coupling at $915 \mathrm{MHz}$ is approximately $-4 \mathrm{~dB}$, and the corresponding value for $2.45 \mathrm{GHz}$ is approximately $-10 \mathrm{~dB}$. When powering sensors embedded in reinforced concrete, the coupling between antennas should be maximized in order to achieve optimum performance. Therefore, $915 \mathrm{MHz}$ is more suitable for power transmission through near field coupling. 


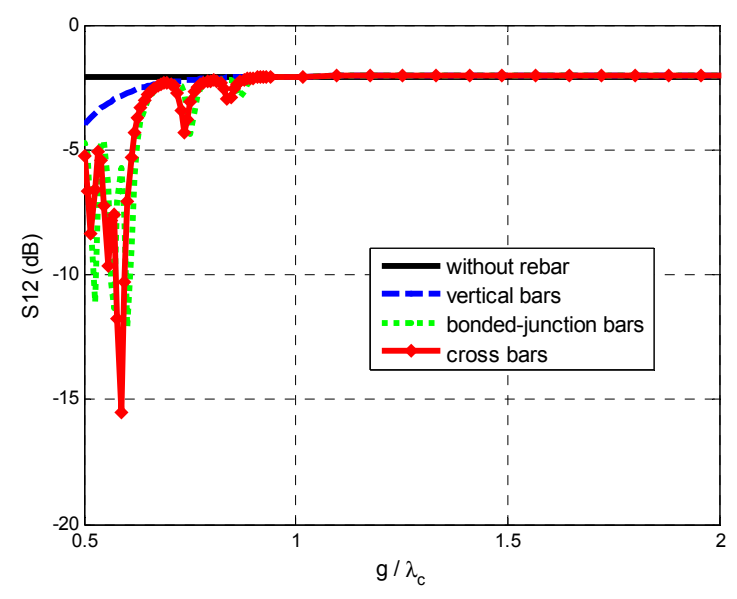

(a) $433 \mathrm{MHz}$.

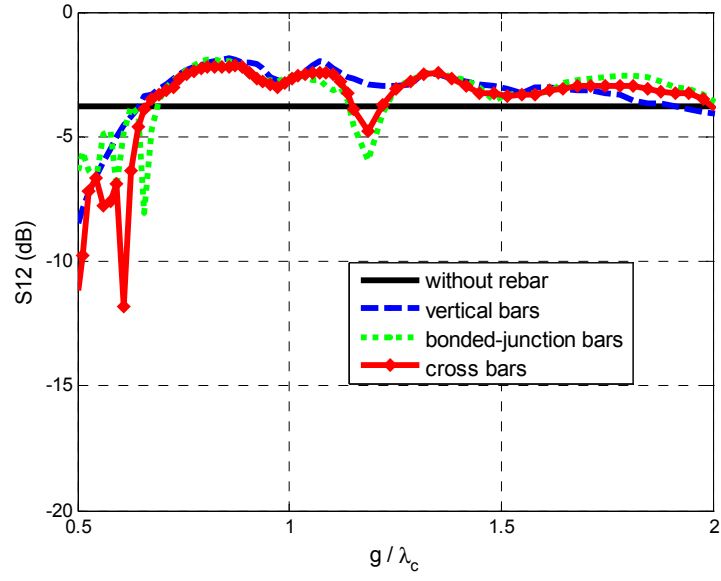

(b) $915 \mathrm{MHz}$.

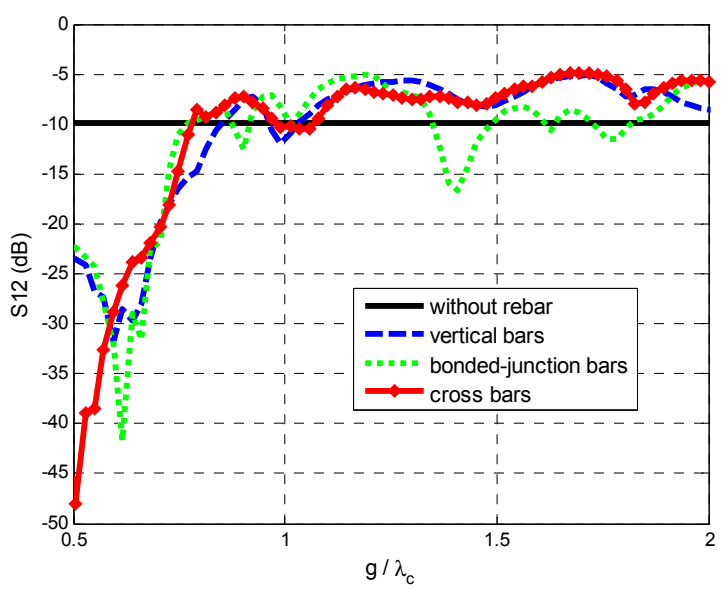

(c) $2.45 \mathrm{GHz}$.

Figure 5.14: Patches coupling for three rebar types.

\subsection{Summary}

Optimum rectennas were designed in this chapter for wireless power transmission to sensors embedded in plain and reinforced concrete structures. The performances of various antenna types were analyzed in free space, dry concrete, saturated concrete and reinforced concrete. Embedded patch antennas are proven to be more suitable for powering sensors embedded in plain and reinforced concrete since they offer larger coupling and they are less sensitive to the changes of concrete's humidity conditions and 
rebars' configurations. Stripline antennas working at $915 \mathrm{MHz}$ were developed for operation in reinforced concrete with different humidity conditions. Matched rectification circuits were also designed to improve the power conversion efficiency. Finally, it can be concluded that in order to design optimum wirelessly powered sensors for plain and reinforced concrete, the operational frequency, antenna type, antenna's position inside concrete, and rectification circuit type should be carefully considered. 


\section{CHAPTER 6}

\section{CONCLUSIONS AND FUTURE WORK}

\subsection{Conclusions}

This dissertation presented a comprehensive analysis of wireless power transmission of sensors embedded in plain and reinforced concrete structures for SHM applications. An air-to-concrete plane wave formulation was developed to calculate the transmission loss, propagation loss and total loss of normal incidence and oblique incidence of electromagnetic wave penetrating through plain concrete half-space and slab. An extended Debye model was also used to describe the electromagnetic properties of concrete. Our results illustrated that the power loss is closely related to the humidity conditions of concrete, operational frequency and incidence angle of the electromagnetic waves. When an EM wave is normally incident into plain concrete at frequencies between 20 and $80 \mathrm{MHz}$, sensors embedded in medium depths inside concrete will receive significantly larger power than other cases we examined. Therefore, sensors embedded in plain concrete in this optimum frequency range will be able to efficiently harvest signals in the bands of shortwave radio and VHF TV.

The propagation from air to reinforced concrete model was also investigated in Ansoft HFSS to characterize the reflection and transmission properties of reinforced concrete slab, which both depend on the geometry of the concrete slab and the configuration of the rabars. The effects of the humidity condition and thickness of the concrete slab were analyzed. Four configuration parameters of the rebars were considered, including the mesh period, the rebar diameter, the rebar depth and the mesh layer offset. 
Our results illustrated that when EM waves are normally incident into a reinforced concrete slab in the frequency range of $902-928 \mathrm{MHz}$, which corresponds to the ISM band, maximum power transmission is achieved. Also, it was shown that in this ISM band, wireless power transmission to sensors embedded in reinforced concrete is less sensitive to variations of the rebar configurations and concrete humidity conditions.

Furthermore, the performance of the dipole and patch antennas was investigated in free space, dry concrete, wet concrete and reinforced concrete. Patch antennas exhibited better efficiency for both wireless power transmission and harvesting. Our simulation results illustrated that patch antennas operating at $70 \mathrm{MHz}$ inside plain concrete can harvest the maximum amount of power. Also, stripline antennas were designed at $915 \mathrm{MHz}$ for sensors embedded in reinforced concrete structures. These antennas were proven to be less sensitive to variations of concrete's humidity conditions and rebar configurations. The coupling between two patch antennas at three typical ISM frequencies, i.e. $433 \mathrm{MHz}, 915 \mathrm{MHz}$ and $2.45 \mathrm{GHz}$ were also studied for power transmission through reinforced concrete. It was found that the antenna coupling at 915 $\mathrm{MHz}$ was strongest and therefore, most appropriate for power transmission of sensors embedded inside reinforced concrete.

Various rectification circuits were also simulated in Ansoft NEXXIM including peak detectors, voltage doublers and multi-stage rectifiers. Matching circuits were also designed to improve the wireless powering efficiency of our system. Moreover, the charging times for typical sensors based on the efficiencies of our systems were calculated. 
In conclusion, the wireless power transmission/harvesting systems that we developed exhibited larger efficiencies than existing solutions. For plain concrete applications, such as sensor monitoring systems for motorways/roads and airstrips, existing wireless recharging systems rely on fixed power supply sources. However, our design focuses on RF power harvesting. Since our systems do not require the installation of sources, significant saving in both time and cost can be achieved. Furthermore, for reinforced concrete applications, such as sensor monitoring systems for bridges/overpasses, concrete walls, dams and parking structures, few inductive coupling solutions were proposed by previous work. On the contrary, our designs are based on antenna coupling at $915 \mathrm{MHz}$, which is suitable for both far-field and near-field wireless power transmission applications. Our work also showed that antennas working in the 902-928 MHz ISM band have less power transmission loss and are less sensitive to variations in concrete's humidity and the configurations of the rabars than other frequencies that were examined.

\subsection{Future Work}

This research was focused on optimization of wireless powering of sensors embedded in concrete for SHM applications. However, our work can be expanded by further research.

Our work focused on applications with basic reinforced structures, such as concrete walls and bridge decks, for which two layers cross/vertical bars are used. This research can be extended to other applications where more complex reinforced concrete structures are involved, such as dams or foundations. 
When designing wireless power transmission and/or harvesting systems for embedded sensors, other types of antennas and rectification circuits can be investigated based on our analysis approach to further maximize the power delivered to the sensors' rechargeable batteries. For example, intelligent reconfigurable antennas could be developed to self-adapt to different applications (such as, concrete monitoring during construction/curing, and health monitoring of structures after they are built) and different concrete environments (various concrete types in terms of humidity, consistency and rebar configuration). Also, CMOS based multi-stage rectifiers could be utilized to increase the RF to DC conversion efficiency and decrease the rectenna size.

Our work focused on analytical and computational methods. Experimental work could be done based on our approach to implement the wireless powering systems for SHM sensors. For example, RF power harvesting systems can be investigated within the 20-80 MHz band for sensors embedded in plain concrete. Wireless power transmission systems can also be implemented within the 902-928 MHz ISM band for sensors embedded in reinforced concrete structures.

Our work focused on maximizing the power transmission efficiency and minimizing the battery charging time of embedded SHM sensors. This research can be extended to the optimization of systems involve communication with totally passive sensors without batteries (i.e., embedded RFID tags with sensory functions for concrete panel's life cycle evolution monitoring). 


\section{REFERENCES}

[1] S. D. Glaser, H. Li, M. L. Wang, J. Ou, and J. Lynch, "Sensor Technology Innovation for the Advancement of Structural Health Monitoring: A Strategic Program of US-China Research for the Next Decade," Smart Structures and Systems, vol. 3, no. 2, pp. 221-244, 2007.

[2] J. T. Bernhard, K. Hietpas, E. George, D. Kuchma, and H. Reis, "An Interdisciplinary Effort to Develop a Wireless Embedded Sensor System to Monitor and Assess Corrosion in the Tendons of Prestressed Concrete Girders," IEEE Topical Conference on Wireless Communication Technology, pp.241-243, 2003.

[3] C. R. Farrar and K. Worden, "An Introduction to Structural Health Monitoring," Philosophical Transactions of the Royal Society, vol. 365, pp. 303-315, 2007.

[4] H. C. Rhim, "Condition Monitoring of Deteriorating concrete Dams using Radar," Cement and Concrete Research, no. 31, pp. 363-373, 2001.

[5] De. Roover, C. Vantomme, and J. Wastieles, "Deformation Analysis of a Modular Connection System by Digital Image Correlation," Experimental Techniques. pp. 37-40. 2002.

[6] P. Klinkhachorn, A. S. Mercer, U. B. Halabe, and H. G. Rao, "An Autonomous Unmanned Ground vehicle for Non-Destructive Testing of Fiber-Reinforced Polymer Bridge Decks," IEEE Instrumentation \& Measurement Magazine, pp. 28-33, Jun. 2007.

[7] H. Song and V. Sarawathy, "Corrosion Monitoring of Reinforced Cncrete Structures- A Review," International Journal of Electrochemical Science, no. 2, pp. 1-28, 2007.

[8] F. Bastianini, F. Matta, A. Rizzo, N. Galati, and A. Nanni, "Overview of Recent Bridge Monitoring Applications using Distributed Brillouin Fiber Optic Sensors," The E-Journal \& Exhibition of Nondestructive Testing, pp. 269-276, 2007.

[9] H. Sohn and C. R. Farrar, "Statistical Process Control and Projection Techniques for Damage Detection," European COST F3 Conference on System Identification and Structural Health Monitoring, pp.105-114, 2000.

[10] G. Naldi and P. Venini, "Postprocessing Singular Solutions by the Wavelet Transform," Structural Damage Assessment Using Advanced Signal Processing Procedures, Proceedings of DAMAS ‘97, pp. 109-120, 1997. 
[11] C. Biemans, W. J. Staszewski, C. Boller, and G. R. Tomlinson, "Crack Detection in Metallic Structures Using Piezoceramic Sensors," Damage Assessment of Structures, Proceedings of the International Conference on Damage Assessment of Structures, pp. 112-121, 1999.

[12] H. Sohn, C. R. Farrar, R. M. Hemez, D. D. Shunk, D. W. Stinemates, B. R. Nadler, and J. J. Czarnecki, "A Review of Structural Health Monitoring Literaure: 1996-2001,” Los Alamos National Laboratory Report, 2004.

[13] C. H. Loh and C. C. Huang, "Damage Identification of Multi-Story Steel Frames Using Neural Networks," Structural Health Monitoring, pp. 390-399, 2000 .

[14] M. Krawczuk, W. Ostachowicz, and G. Kawiecki, " Detection of Delamination in Cantilevered Beams Using Soft Computing Methods," European COST F3 Conference on System Identification and Structural Health Monitoring, Madrid, Spain, pp. 243-252, 2000.

[15] K. Worden and A. J. Lane, "Damage Identification Using Support Vector Machines," Smart Materials and Structures, vol. 10, pp. 540-547, 2001

[16] L. L. Lapin, "Probability and Statistics for Modern Engineering," PWS-Kent Publishing, 2nd Edition, 1990.

[17] Y. Q. Ni, K. Y. Wong, and Y. Xia, "Health Checks through Landmark Bridges to Sky-high Structures," Advances in Structural Engineering, vol. 14, no. 1, pp. 103-119, 2011.

[18] A. Gastineau, T. Johnson, and A. Schultz, "Bridge Health Monitoring and Inspections-A Survey of Methods,” Report MN/RC 2009-29, 2009.

[19] S. Kim, "Wireless Sensor Networks for Structural Health Monitoring," Research project, 2005.

[20] F. Akyildiz, W. Su, Y. Sankarasubramaniam, and E. Cayirci, "Wireless Sensor Networks: A Survey," Computer Networks, vol. 38, pp. 393-422, 2002.

[21] J. Anderson, R. Szewczyk, A. Mianwaring, D. Culler, and J. Anderson, "Analysis of Wireless Sensor Networks for Habitat Monitoring," Wireless Sensor Networks, 2004.

[22] V. Snayder, B. Chen, K. Lorincz, T. Jones, and M. Welsh, "Sensor Networks for Medical Care," Technical report, Harvard University, 2005. 
[23] D. J. Cook and S. K. Das, "How Smart are Our Environments? An Updated Look at the State of the Art," Pervasive and Mobile Computing, vol. 3, no. 2, pp. 53-73, 2007.

[24] J. Heidemann, W. Ye, J. Wills, A. Syed, and Y. Li, "Research Challenges and Applications for Underwater Sensor Networking," Proceedings of IEEE Wireless Communications and Networking Conference, 2006.

[25] P. Soontornpipit, C. M. Furse, Y. C. Chung, and B. M. Lin, "Optimization of a Buried Microstrip Antenna for Simultaneous Communication and Sensing of Soil Moisture," IEEE Transactions on Antennas and Propagation, vol. 54, no. 3, pp.797-800, Mar. 2006.

[26] J. Kim and Y. Rahmat-Samii, "Implanted Antenna inside a Human Body: Simulations, Designs and Characterizations," IEEE Transactions on Microwave Theory and Techniques, vol. 52, no. 8, pp.1934-1943, Aug. 2004.

[27] D. Wang and W. Liao, "Wireless Transmission for Health Monitoring of Large Structures," IEEE Transactions on Instrumentation and Measurement, vol. 55, no. 3, pp. 972-981, Jun. 2006.

[28] N. Xu, S. Rangwala, K. K. Chintalapudi, D. Ganesan, A. Broad, R. Govindan, and D. Estrin, "A Wireless Sensor Network for Structural Monitoring," Proceedings of the $2^{\text {nd }}$ International Conference on Embedded Networked Sensor Systems, Nov. 2004.

[29] J. M. Engel, L. Zhao, Z. Fan, J. Chen, and C. Liu, "Smart Brick - A Low Cost, Modular Wireless Sensor for Civil Structure Monitoring," International Conference on Computing, Communications and Control Technologies, Aug. 2004.

[30] B. Carkhhuff and R. Cain, "Corrosion Sensors for Concrete Bridges," IEEE Instrumentation and Measurement Magazine, pp. 19-24, Jun. 2003.

[31] G. Jolly and M. Younis, "An Energy-efficient, Scalable and Collision Less MAC Layer Protocol for Wireless Sensor Networks," Wireless Communications and Mobile Computing, vol. 5, no. 3, pp. 285-304, 2005.

[32] B. A. Warneke, M. D. Scott, B. S. Zhou, and K. S. J. Pister, "An Autonomous 16 $\mathrm{mm}^{3}$ Solar-Powered Node for Distributed Wireless Sensor Networks," Proceedings of IEEE Sensors, vol. 2, pp. 1510-1515, 2002. 
[33] M. Stordeur and I. Stark, "Low Power Thermoelectric Generator - Self-Sufficient Energy Supply for Micro Systems," $16^{\text {th }}$ International Conference on Thermoelectrics, pp. 575 - 577, 1997.

[34] H. Glosch, M. Ashauer, U. Pfeiffer, and W. Lang, "A Thermoelectric Converter for Energy Supply," Sensors and Actuators, vol. 74, pp. 246-250, 1999.

[35] N. S. Shenck and J. A. Paradiso, "Energy Scavenging with Shoe-Mounted Piezoelectric," IEEE Micro, vol. 21, pp. 30-41, 2001.

[36] A. C. Patel, M. P. Vaghela, H. Bajwa, and P. K. Patra, "Power Harvesting for Low Power Wireless Sensor Network," Loughborough Antenna and Propagation Conference, pp. 633-635, 2009.

[37] P. Scholz, C. Reinhold, J. Werner, and U. Hilleringmann, "Analysis of Energy Transmission for Inductive Coupled RFID Tags," IEEE International Conference on RFID, Mar. 2007.

[38] A. Karalis, J. D. Joannopoulos, and M. Soljacic, "Efficient Wireless NonRadiative Mid-Range Energy Trnasfer," Annals of Physics, vol 323, pp. 34-48, 2008 .

[39] T. Le, K. Mayaram, and T. Fiez, "Efficient Far-Field Radio Frequency Energy Harvesting for Passively Powered Sensor Networks," IEEE Journal of Solid State Circuits, vol. 43, no. 5, pp. 1287-1302, May 2008.

[40] E. Sazonov, H. Li, D. Curry, and P. Pillay, "Self-Powered Sensors for Monitoring of Highway Bridges," IEEE Sensors Journal, vol. 9, pp. 1422-1429, 2009.

[41] M. Wang, "Embedded Strain Sensor with Power Scavenging from Bridge Vibration," Master thesis, 2004.

[42] P. Soontornpipit, C. M. Furse, and C. C. You, "Design of Implantable Microstrip Antenna for Communication with Medical Implants," IEEE Transactions on Microwave Theory and Techniques, vol. 52, no. 8, pp. 1944-1951, Aug. 2004.

[43] EmbedSence, Wireless Sensor, Microstrain: http://www.microstrain.com/embedSense.aspx.

[44] M. M. Andringa, D. P. Neikirk, N. P. Dickerson, and S. L. Wood, "Unpowered Wireless Corrosion Sensor for Steel Reinforced Concrete," IEEE Sensors, pp. 155-158. Nov. 2005. 
[45] P. Merlino and A. Abramo, "An Integrated Sensing/Communication Architecture for Structural Health Monitoring," IEEE Sensors Journal, vol. 9, no. 11, pp. 1397-1404, Nov. 2009.

[46] A. Kurs, A. Karalis, R. Moffatt, J. D. Joannopoulos, P. Fisher, and M. Soljacic, "Wireless Power Transfer via Strong Coupled Magnetic Resonances," Science, Vol. 317, No.5834, pp. 83-86, Jul. 2007.

[47] A. Kurs, R. Moffatt, and M. Soljacic, "Simultaneous Mid-range Power Transfer to Multiple Devices," Applied Physics Letters, vol. 96, 2010.

[48] O. Jonah and S. V. Georgakopoulos, "Wireless Power Transmission to Sensors Embedded in Concrete via Magnetic Resonance," IEEE $12^{\text {th }}$ Annual Wireless and Microwave Technology Conference, pp. 1-6, 2011.

[49] K. M. Farinhold, G. Park, and C. R. Farrar, "RF Energy Transmission for a LowPower Wireless Impedance Sensor Node," IEEE Sensors Journal, vol. 9, no. 7, pp.793-800, Jul. 2009.

[50] D. Mascarenas, E. Flynn, and M. Todd, "Wireless Sensor Technologies for Monitoring Civil Structures," Sound and Vibration, 2008.

[51] K. M. Z. Shams and M. Ali, "Wireless Power Transmission to a Buried Sensor in Concrete,” IEEE Sensors Journal, vol. 7, no. 12, pp. 1573-1577, Dec. 2007.

[52] M. Ali, G. Yang, and R. Dougal, "A New Circularly Polarized Rectenna for Wireless Power Transmission and Data Communication," IEEE Antennas and Wireless Propagation Letters, vol. 4, pp. 205-208, 2005.

[53] M. Ali, G. Yang, and R. Dougal, "Miniature Circularly Polarized Rectenna with Reduced Out-of-Band Harmonics," IEEE Antennas and Wireless Propagation Letters, vol. 5, pp. 107-110, 2006.

[54] K. M. Z. Shams, M. Ali, and A. M. Miah, "Characteristics of an Embedded Microstrip Patch Antenna for Wireless Infrastructure Health Monitoring," IEEE Antennas and Propagation Society International Symposium, Jul. 2006.

[55] X. Jin and M. Ali, "Reflection and Transmission Properties of Embedded Dipoles and PIFAs inside Concrete at $915 \mathrm{MHz}$," IEEE Antennas and Propagation Society International Symposium, Jun. 2009. 
[56] Long-term Plan for Concrete Pavement Research and Technology-The Concrete Pavement Road Map: An Ececutive Summary, FHWA Puablication Number: GHWA-HRT-05-053, Sep. 2005.

[57] W. J. McCarter, G. Starrs, and T. M. Chrisp, "Immittance Spectra for Portland Cement/Fly Ash-based Binders during Early Hydration," Cement and Concrete Research, vol. 29, pp. 377-387, 1999.

[58] O. Buyukozturk, "Electromagnetic Properties of Concrete and Their Significance in Nondestructive Testing," Transportation Research Record, no. 1574, pp. 10-17, 1997.

[59] T. Bourdi, J. E. Rhazi, F. Boone, and G. Ballivy, "Application of Jonscher Model for the Characterization of the Dielectric Permittivity of Concrete," Journal of Physics D:Applied Physics, vol. 41, no. 20, 1-9, Oct. 2008.

[60] L. Sandrolini, U. Reggiani, and A. Ogunsola, "Modeling the Electrical Properties of Concrete for Shielding Effectiveness Prediction," Journal of Physics D: Applied Physics, vol. 40, pp. 5366-5372, 2007.

[61] C. Balanis, Antenna Theory: Analysis and Design, Third Ed., NJ: John Wiley, 2005 .

[62] D. M. Pozar, Microwave Engineering, Third Ed, NJ: John Wiley, 2005.

[63] K. G. Ayappa and H. T. Davis, "Microwave Heating: An Evaluation of Power Formulations," Chemical Engineering Science, vol. 46, no.4, 1005-1016, 1991.

[64] P. Soontornpipit, C. M. Cynthia, and Y. C. Chung, "Miniaturized Biocompatible Microstrip Antenna Using Genetic Algorithm," IEEE Transactions on Antennas and Propagation, vol. 53, no. 6, pp. 1939-1945, Jun. 2005.

[65] A. Rosen, M. A. Stuchly, and A. V. Vorst, "Applications of RF/Mocrowaves in Medicine," IEEE Transactions on Microwave Theory and Techniques, vol. 50, no. 3, pp. 963-974, Mar. 2002.

[66] L. Liu, S. Zhou, and J. Cui, "Prospects and Problems of Wireless Communications for Underwater Sensor Networks," Wireless Communication and Mobile Computing, vol. 8, pp. 977-994, 2008.

[67] J. B. Hasted, “Aqueous Dielectrics," Chapman and Hall, New York, 1973. 
[68] T. Shaneyfelt, M. A, Joordens, K. Nagothu, and M. Jamshidi, "RF Communication between Surface and Underwater Robotic Swarms," Automation Congress, Dec. 2008.

[69] G. Benelli, A. Pozzebon, and G. Ragseo, "An RFID Based System for the Unverwater Tracking of Pebbles on Artificial Coarse Beaches," IEEE 2009 Third International Conference on Sensor Technologies and Applications, pp. 294-300, Jun. 2009.

[70] Z. Sun and I. F. Akyildiz, "Magnetic Induction Communications for Wireless Underground Sensor Networks," IEEE Transactions on Antennas and Propagation, Vol. 58, No. 7, pp. 2426-2435, Jul. 2010.

[71] A. R. Silva and M. C. Vuran, "Communication with Aboveground Devices in Wireless Underground Sensor Networks: An Empirical Study," IEEE International Conference on Communications, pp. 1-6, 2010.

[72] C. Ritsema, etal, "A New Wireless Underground Network System for Continuous Monitoring of Soil Water Contents," Water Resources Research Journal, vol. 45, pp. 1-9, May 2009.

[73] C. P. Lin, "Frequency Domain versus Travel Time Analyses of TER Waveforms for Soil Moisture Measurements," Soil Science Society of America Journal, vol. 67, no. 3, pp. 720-729, 2003.

[74] R. A. Dalke, C. L. Holloway, P. McKenna, M. Johansson, and A. S. Ali, "Effects of Reinforced Concrete Structures on RF Communications," IEEE Transactions on Electromagnetic Compatibility, vol. 42, no. 4, pp. 486-496, Nov. 2000.

[75] M. Dehmollaian and K. Sarabandi, "An Approximate Solution of Scattering from Reinforced Concrete Walls," IEEE Transactions on Antennas and Propagation, vol. 56, no. 8, pp. 2681-2690, Aug. 2008.

[76] D. Pena, R. Feick, H. Hristov, and W. Grote, "Measurement and Modeling of Propagation Losses in Brick and Concrete Walls for the 900-MHz Band," IEEE Transaction on Antennas and Propagation, vol. 51, no. 1, pp. 31-39, Jan. 2003.

[77] E. Richalot, M. Bonilla, M. Wong, V. Fouad-Hanna, H. Baudrand, and J. Wiart, "Electromagnetic Propagation into Reinforced-Concrete Walls," IEEE Transactions on Microwave Theory and Techniques, vol. 48, no. 3, pp. 357-366, Mar. 2000.

[78] http://www.ansoft.com/products/hf/hfss. 
[79] User's Guide-High Frequency Structure Simulator: jpkc.xmu.edu.cn/dccyw bjssy/innovation/HFSS/Ansoft-Hfss\%20Users'\%20Guide.pdf

[80] X. Jin and M. Ali, "Embedded Antennas in Dry and Saturated Concrete for Application in Wireless Sensors," Progress in Electromagnetics Research, PIER 102, pp. 197-211, 2010.

[81] J. D. Kraus and R. J. Marhefka, Antennas for All Applications, Third Ed, 2002, McGraw-Hill Higher Education.

[82] I. J. Bahl and S. S. Stuchly, "Analysis of a Microstrip Covered with a Lossy Dialectric," IEEE Transactions on Microwave Theory and Techniques, vol. 28, no. 2, pp. 104-109, Feb. 1980.

[83] K. M. Luk and W. Y. Tam, "Microstrip Antennas Covered with Dielectric Materials," IEEE Antennas and Propagation Society International Symposium, July. 1989.

[84] D. M. Dobkin, “The RF in RFID: Passive UHF RFID in Practice," Elsevier Inc, 2008 .

[85] Agilent HSMS-2820 Schottky Detector Diodes Data Sheet.

[86] D. W. Harrist, "Wireless Battery Charging System Using Radio Frequency Energy Harvesting,” Master Thesis, 2001.

[87] G. Park, T. Rosing, M. D. Todd, C. R. Farrar, and W. Hodgkiss, "Energy Harvesting for Structural Health Monitoring Sensor Networks," Journal of Infrastructure Systems, pp.64-79, 2008.

[88] N. G. Elvin, N. Lajnef, and A. A. Elvin, "Feasibility of Structural Monitoring with Vibration Powered Sensors," Smart Material Structure, vol.15, pp. 977-986, 2006.

[89] H. A. Sodano, G. E. Simmers, R. Dereux, and D. J. Inman, "Recharging Batteries using Energy Harvested from Thermal Gradients," Journal of Intelligent Material Systems and Structural, Oct. 2006.

[90] P. Ruetschi, F. Meli, and J. Desilvestro, "Nickel-metal Hydride Batteries. The Preferred Batteries of the Future?" Journal of Power Sources, vol. 57, pp. 85-91, 1995. 
[91] A. Mianwaring, J. Polastre, R. Szewczyk, D. Culler, and J. Anderson, "Wireless Sensor Networks for Habitat Monitoring," ACM Workshop on Sensor Networks and Applications, 2002. 
VITA

\section{SHAN JIANG}

1983

Born, Liaoning, China

2005 B. E., Electric Information Science and Technology

Tianjin Polytechnic University

Tianjin, China

2008

M. S., Signal and Information Processing

Tianjin Polytechnic University

Tianjin, China

2011

Ph. D. Candidate, Electrical Engineering

Florida International University

Miami, USA

\section{PUBLICATIONS AND PRESENTATIONS}

\section{JOURNALS}

[1] Shan Jiang and Georgakopoulos, S.V. "Optimum Wireless Powering of Sensors Embedded in Concrete", IEEE Transactions on Antennas and Propagation. [accepted]

[2] Shan Jiang and Georgakopoulos, S.V. "Electromagnetic Wave Propagation into Fresh Water", Journal of Electromagnetic Analysis and Applications, vol. 3, no. 7, pp. 261-266, 2011.

[3] Yuecun Wang, Shan Jiang, and Tu Xiong, "Application of Ray Tracing Algorithm on the Fabric CAD", Journal of Textile Research, vol.28, No.8, pp.113-116, 2007

[4] Shan Jiang and Yuecun Wang, "Denoising Algorithm based on Median Filter and Morphology”, Instrumentation Customer, vol.14, No.4, pp.106-107, 2007.

\section{CONFERENCES}

[1] Georgakopoulos, S.V. and Shan Jiang, "Wireless Power Transmission for Underground Sensors", IEEE Antennas and Propagation Society International Symposium (APS/URSI 2011), Spokane, WA, Jul. 2011.

[2] Shan Jiang and Georgakopoulos, S.V. "Optimum Wireless Power Transmission 
from Air to Lossy Meida", Wireless and Microwave Technology Conference (WAMICON), 2011 IEEE 12th Annual, Clearwater, FL, Apr. 2011.

*Presented by Shan Jiang

[3] Shan Jiang and Georgakopoulos, S.V. "Optimum Wireless Power Transmission through Reinforced Concrete Structure", IEEE International Conference on RFID, Orlando, FL, Apr. 2011.

*Presented by Shan Jiang

[4] Shan Jiang and Georgakopoulos, S.V. "Optimum Power Transmission of Wireless Sensors Embedded in Concrete", IEEE International Conference on RFID, Orlando, FL, Apr. 2010.

*Presented by Shan Jiang

[5] Georgakopoulos, S.V. and Shan Jiang, "Wireless Powering of Sensors Embedded in Concrete", Wireless and Microwave Technology Conference (WAMICON), 2010 IEEE 11th Annual, Melbourne, FL, Apr. 2010.

*Presented by Shan Jiang

[6] Shan Jiang and Georgakopoulos, S.V. "Wireless Powering of Sensors Embedded in Concrete", IEEE Antennas and Propagation Society International Symposium (APS/URSI 2009), Charleston, SC, Jun. 2009.

*Presented by Shan Jiang 